\title{
Assessment of LTCC-Based Dielectric Flat Lens Antennas and Switched-Beam Arrays for Future 5G Millimeter-Wave Communication Systems
}

\author{
Marc Imbert ${ }^{\circledR}$, Student Member, IEEE, Jordi Romeu, Fellow, IEEE, Mariano Baquero-Escudero, Member, IEEE, \\ Maria-Teresa Martinez-Ingles, Jose-Maria Molina-Garcia-Pardo, and Lluis Jofre, Fellow, IEEE
}

\begin{abstract}
This paper presents the design, low-temperature co-fired ceramics (LTCC) fabrication, and full experimental verification of novel dielectric flat lens antennas for future high data rate $5 \mathrm{G}$ wireless communication systems in the $60 \mathrm{GHz}$ band. We introduce and practically completely evaluate and compare the performance of three different inhomogeneous gradient-index dielectric lenses with the effective parameters circularly and cylindrically distributed. These lenses, despite their planar profile antenna configuration, allow full 2 -D beam scanning of high-gain radiation beams. A time-domain spectroscopy system is used to practically evaluate the permittivity profile achieved with the LTCC manufacturing process, obtaining very good results to confirm the viability of fabricating inhomogeneous flat lenses in a mass production technology. Then, the lenses performance is evaluated in terms of radiation pattern parameters, maximum gain, beam scanning, bandwidth performance, efficiencies, and impedance matching in the whole frequency band of interest. Finally, the performance of the three lenses is also experimentally evaluated and compared to a single omni-directional antenna and to a ten-element uniform linear array of omni-directional antennas in real $60 \mathrm{GHz}$ wireless personal area network indoor line-of-sight (LOS) and obstructed-LOS environments, obtaining interesting and promising remarkable results in terms of measured received power and root-mean-square delay spread. At the end of this paper, an innovative switched-beam antenna array concept based on the presented cylindrically distributed effective parameters lens is also introduced and completely evaluated, confirming the potential applicability of the proposed antenna solution for future 5G wireless millimeter-wave communication system.
\end{abstract}

Index Terms-5G, $60 \mathrm{GHz}$ band, beam steering, delay spread, flat lens antennas, inhomogeneous lenses, low-temperature

Manuscript received September 21, 2016; revised October 9, 2017; accepted October 19, 2017. This work was supported in part by the Spanish InterMinisterial Commission on Science and Technology (CICYT) under Project TEC2013-47360-C3-1-P and Project TEC2013-47360-C3-2-P and in par by the "Ministerio de Economía" through the FPI Fellowship Program. (Corresponding author: Marc Imbert.)

M. Imbert, J. Romeu, and L. Jofre are with the AntennaLab Research Group, Department of Signal Theory and Communications, Universitat Politècnica de Catalunya, 08034 Barcelona, Spain (e-mail: marc.imbert@tsc.upc.edu).

M. Baquero-Escudero is with the Instituto de Telecomunicaciones y Aplicaciones Multimedia, Universitat Politècnica de València, 46022 València, Spain (e-mail: mbaquero@dcom.upv.es).

M.-T. Martinez-Ingles is with the University Center of Defense, San Javier Air Force Base, MDE-UPCT, 30720 Murcia, Spain.

J.-M. Molina-Garcia-Pardo is with the Departamento de Tecnologías de la Información y las Comunicaciones, Universidad Politécnica de Cartagena, 30202 Murcia, Spain (e-mail: josemaria.molina@upct.es).

Color versions of one or more of the figures in this paper are available online at http://ieeexplore.iee.org.

Digital Object Identifier 10.1109/TAP.2017.2767821 co-fired ceramics (LTCC), millimeter-wave antennas, power delay profile (PDP), smart antennas, switched-beam arrays, wireless personal area network (WPAN).

\section{INTRODUCTION}

$\mathbf{T}$ HE future broadband wireless communication systems will have the need for more bandwidth in order to satisfy the increasing demands to achieve higher data rates. In this sense, the millimeter-wave frequency band will play a key role in fifth generation (5G) wireless cellular networks [1]-[3].

Four different frequency bands around 28, 38, 60, and $73 \mathrm{GHz}$ have been considered in the millimeter-wave region as perfect candidates for future $5 \mathrm{G}$ mobile communication systems in both indoor and outdoor environments [3], [4]. Actually, wireless personal area networks (WPANs) for highspeed data rate short-range communications around $60 \mathrm{GHz}$ band (from 57 to $64 \mathrm{GHz}$ in the United States, and up to $66 \mathrm{GHz}$ in Europe [5]), have attracted growing attention from the scientific community and industry in the last years. This huge amount of bandwidth available could allow the development of high throughput transmission systems for the future $5 \mathrm{G}$ cellular networks. However, at millimeter-wave frequencies, the path loss in free-space propagation is considerably higher than at lower microwave frequencies (for example, the attenuation is up to $28 \mathrm{~dB}$ higher at $60 \mathrm{GHz}$ compared to at $2.45 \mathrm{GHz}$, for a fixed transmission distance). Therefore, in order to allow future $5 \mathrm{G}$ millimeter-wave devices to achieve high data rate wireless transmissions, from the antenna point of view, it is absolutely necessary to dispose of high-directive antennas to overcome the aforementioned huge path loss attenuation. Additionally, antennas with certain beam-steering capabilities are also desirable in order to facilitate the reconfiguration of the radiation beam in situations of transmission blockage between devices in line-of-sight (LOS), obstructedLOS (OLOS), or even in non-LOS (NLOS). Moreover, beamsteerable adaptive antennas for $5 \mathrm{G}$ systems are not yet conveniently available at most millimeter-wave frequencies, even for researchers in order to measure and characterize the channel at a wide frequency range [6].

So far, many types of antenna structures have been proposed for millimeter-wave wireless communication systems around $60 \mathrm{GHz}$ frequency band [7], most of them based on the complex phased-array antenna concept. With this antenna solution, 
high-gain radiation beams can be scanned in two-dimensions at a fast rate. However, they require a difficult integration of some complex, lossy, and bulky components such solid-state phase shifters, making this antenna alternative very expensive at high frequencies for consumer mobile devices.

Aperture antennas, such as profiled lenses, rectangular or conical horns, and reflectors are traditional antenna solutions at millimeter-wave frequencies for communications, radar, and imaging applications due to their high gain and wide bandwidth. However, most common apertures with beamscanning capabilities result in a large and volumetric antenna configuration not suitable for consumer mobile devices (e.g., a homogeneous profiled lens illuminated by a conical horn antenna with a mechanical system to steer the radiation beam in two-dimensions [8]), or their planar implementation allow only 1-D beam steering, instead of 2-D.

Consequently, in [9], we introduced a planar profile antenna configuration based on the switched-beam array antenna concept (see [10]) with an inhomogeneous gradient-index dielectric flat lens to steer and enhance the radiation in a specific direction, achieving a 2-D beam scanning of high-gain radiation beams while maintaining a completely flat antenna profile very suitable for medium-sized mobile devices. The novel inhomogeneous flat lens design used in the switchedbeam antenna array was introduced, fabricated, and electromagnetically characterized in [11].

Therefore, compared to previously published works, in this paper, we introduce design, numerical simulation, novel fabrication in low-temperature co-fired ceramics (LTCC) technology, full experimental verification, and practical application of two new inhomogeneous gradient-index dielectric flat lenses for future high data rate $5 \mathrm{G}$ wireless communication systems in the $60 \mathrm{GHz}$ band. The performance of these lenses, which have their effective dielectric parameters circularly and cylindrically distributed, is also compared to the aforementioned lens presented in [11], in terms of radiation pattern parameters, highest achievable gain, beam-scanning capabilities in both theta and phi dimensions, bandwidth performance, efficiencies, and impedance matching over the whole frequency band of interest. Then, the performance of the three lenses is also experimentally evaluated and compared to a single omni-directional antenna and to a ten-element uniform linear array (ULA) of omni-directional antennas in a real $60 \mathrm{GHz}$ WPAN indoor environment under LOS and OLOS conditions, in terms of measured received power and rootmean-square (RMS) delay spread [15], [16], to evaluate their practical application as smart antenna solutions for high data rate $5 \mathrm{G}$ millimeter-wave systems, not only for mobile devices but also as a possible solution for access points (APs) [17], or even for outdoor base stations (BS), due to their flat antenna configuration and 2-D scanning capability of highgain radiation beams. Finally, in the last section of this paper, we also introduce a new switched-beam antenna array concept based on a novel cylindrically distributed parameters flat lens, which has an effective gradient-index in one axis, while a constant index is maintained along the other one. With this cylindrical effective parameter distribution, the beam scanning can be performed in one plane by moving (or selecting) the

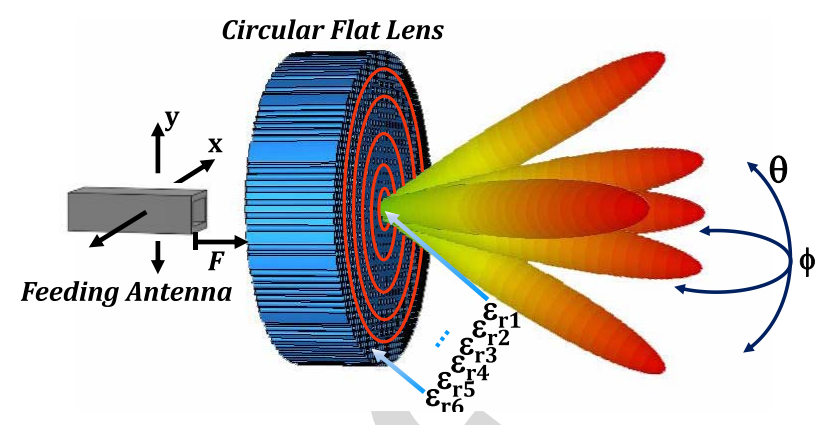

Fig. 1. Circularly distributed parameters flat lens concept and modeling by using triangular unit cells of perforations.

position of a radiating single element along the gradient-index axis, whereas the beam can be maintained invariant in the other direction, in which the effective parameters are kept constant, despite changing the radiating element position in this particular axis. In this way, the beam scanning can be achieved in the constant-index axis of the lens by means of a different technique, a frequency-scanned slot antenna array (FSSA), which it is also introduced at the end of this paper, in order to reduce the switching elements needed in the proposed complete switched-beam antenna array structure, to finally perform the scan of the high-gain radiation beam in both theta and phi dimensions of the space.

\section{Flat Lenses Design and Simulation Results}

Two different new inhomogeneous gradient-index dielectric flat lenses are designed and numerically simulated, each one with its particular effective parameters distribution, in order to obtain two different radiation pattern characteristics and beam-steering capabilities. In this sense, we are interested in achieving two different high-gain beam shapes: a pencil-beam and a fan-beam radiation patterns, because depending on the situation they have been experimentally proved as attractive solutions in the millimeter-wave frequency band for indoor communications [17] and 5G systems [18].

\section{A. Concept Description}

The particular parameters in both lens designs are optimized previously considering the constraints and difficulties in the fabrication of inhomogeneous lenses. Regarding this point, we investigated the possibility of fabricating the designs in a mass production technology such as LTTC technology. Therefore, in the following sections, the concept description and design considerations are defined taking into account the viability in the subsequently prototype fabrication.

1) Circularly Distributed Parameters Flat Lens Concept: The inhomogeneous gradient-index circular flat lens operating principle and design procedure are completely described in [11], and the theoretical concept is depicted in Fig. 1.

Fundamentally, the design consists of a set of six concentric rings of different permittivity $\left(\varepsilon_{r}\right)$ materials, in order to produce the desired phase delays required to obtain a plane wave behind the lens, when the lens is illuminated from its central focus position. In the same way, when the feeding position 


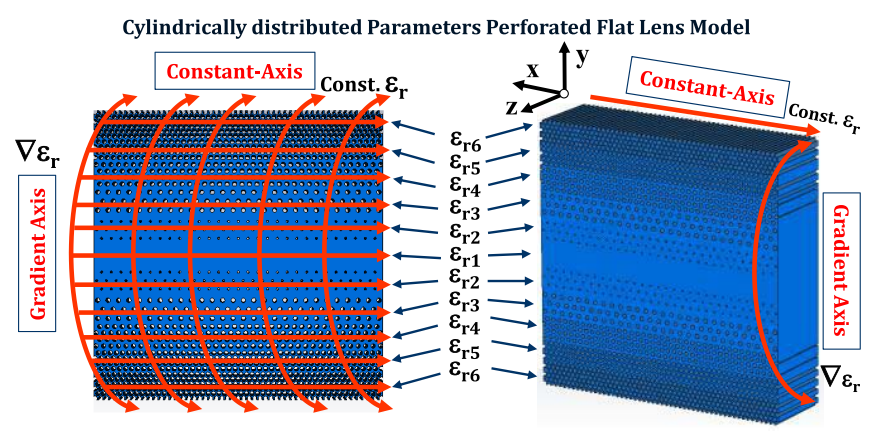

Fig. 2. Cyllindrically distributed parameters flat lens concept and modeling by using triangular unit cells of perforations.

is moved along $y$-or $x$-directions (see Fig. 1), the different permittivity values of the lens produce a linear phase slope that steers the beam, accordingly [11]. Given the lens circular effective parameters distribution, the described behavior is independent of the axis in which the feeding antenna is moved along; the beam will be steered in the same manner.

2) Cylindrically Distributed Parameters Flat Lens Concept: As it has been stated before, a fan-beam radiation pattern (i.e., a beam with a narrow beamwidth in one dimension, broader in the orthogonal) could be very useful for many applications. More specifically, it has been successfully evaluated for high-speed indoor communication systems operating in the $60 \mathrm{GHz}$ band [17], recommending its utilization in certain situations at APs or portable stations (PSs), for example, due to its good immunity to azimuth pointing deviation [17]. Therefore, in order to achieve a fan-beam pattern, a cylindrical lens, to correct the phase of a feeding antenna only in one dimension, in which the beam will be narrower, is needed.

Therefore, in order to achieve a fan-beam radiation pattern, a cylindrical lens, to correct the phase of a feeding antenna only in one dimension, in which the beam will be narrower, is needed. However, it is essential to preserve a planar structure, despite a cylindrical permittivity profile is needed. Hence, the cylindrically distributed parameters lens functioning principle, along its gradient-index axis, is the same as for the previous circular lens described in [11], while in the constantindex axis, the lens is not performing any phase correction, and thus the radiation beam from the source is not being modified. The introduced novel lens achieves the desired behavior at the same time it preserves a planar antenna structure, very interesting for all aforementioned reasons related to APs and PSs.

The cylindrically distributed parameters flat lens concept is depicted in Fig. 2. Fundamentally, consists in a set of eleven rectangular sections of six different permittivity materials, to produce the desired phase delays required to obtain a plane wave, when the lens is illuminated from its central focus position, in the same way as it has been described for the circular lens. Likewise, when the feeding position is moved along $y$-direction (see Fig. 2), the different permittivity values produce a linear phase slope, which steers the beam only along the gradient-index axis (i.e., along $y$-direction), accordingly. As a result of the lens cylindrical parameters distribution,
TABLE I

LTCC PERForated Lenses Characteristic PARAMETERS

\begin{tabular}{|c|c|c|c|c|c|}
\hline Section/Ring & $\begin{array}{c}\text { Section/ } \\
\text { Ring thickness }\end{array}$ & $\boldsymbol{\varepsilon}_{\text {reff }}$ & $\boldsymbol{\alpha}$ & $\boldsymbol{d}$ & $\boldsymbol{s}$ \\
\hline$\varepsilon_{\mathrm{r} 1}$ & $2.27 \mathrm{~mm}$ & 7.1 & - & - & - \\
\hline$\varepsilon_{\mathrm{r} 2}$ & $2.27 \mathrm{~mm}$ & 6.79 & 0.051 & $0.2 \mathrm{~mm}$ & $0.845 \mathrm{~mm}$ \\
\hline$\varepsilon_{\mathrm{r} 3}$ & $2.27 \mathrm{~mm}$ & 6.01 & 0.179 & $0.4 \mathrm{~mm}$ & $0.901 \mathrm{~mm}$ \\
\hline$\varepsilon_{\mathrm{r} 4}$ & $2.27 \mathrm{~mm}$ & 4.99 & 0.346 & $0.4 \mathrm{~mm}$ & $0.648 \mathrm{~mm}$ \\
\hline$\varepsilon_{\mathrm{r} 5}$ & $2.27 \mathrm{~mm}$ & 3.92 & 0.521 & $0.4 \mathrm{~mm}$ & $0.528 \mathrm{~mm}$ \\
\hline$\varepsilon_{\mathrm{r} 6}$ & $2.27 \mathrm{~mm}$ & 2.9 & 0.639 & $0.4 \mathrm{~mm}$ & $0.476 \mathrm{~mm}$ \\
\hline
\end{tabular}

if the position of the feeding element is moved along the constant-index axis (i.e., $x$-direction), the beam is maintained invariant, because the phase is not being corrected in this specific dimension, to finally obtain the desired fan-beam pattern.

\section{B. Practical Dielectric Gradient-Index Flat Lens Design}

After an optimization process, with a tradeoff between the maximum achievable gain and aperture dimensions (gain values greater than $14 \mathrm{~dB}$, or even $20 \mathrm{~dB}$, are required to ensure acceptable system performance and range around $60 \mathrm{GHz}$ band [8], [10]), the theoretical lens total dimensions are fixed in $25 \mathrm{~mm} \times 25 \mathrm{~mm}\left(5 \lambda_{60 \mathrm{GHz}} \times 5 \lambda_{60 \mathrm{GHz}}\right)$, and $25 \mathrm{~mm}$ in diameter, for the cylindrically and circularly distributed parameters lenses, respectively, with $7 \mathrm{~mm}$ thickness $\left(1.4 \lambda_{60} \mathrm{GHz}\right)$, and a focal length of $F=6.25 \mathrm{~mm}\left(1.25 \lambda_{60 \mathrm{GHz}}\right)$, for both lenses.

Applying the functioning principle and design procedure described in [11] for the circular flat lens, and the same principle for the cylindrically distributed parameters flat lens but considering the particularities explained in the previous section, the set of six different permittivity values needed, respectively, for the different six rings or eleven zones of both lenses are obtained and summarized in Table I.

Then, we selected the DuPont 9k7 $\left(\varepsilon_{r}=7 . l, \tan \delta=\right.$ $0.0009)$ dielectric material in order to model, simulate, and fabricate the final LTCC lens prototypes, using an interesting alternative to traditional fabrication methods, which consists in perforating a single layer of dielectric substrate, as it is described in [11]-[14], to reduce its effective dielectric constant. If the diameter of the holes perforated in the substrate $(d)$ and the distance between them $(s)$ are kept smaller than $\lambda_{\text {eff }} / 2$, the substrate will appear to have a uniform effective permittivity. Hence, the set of characteristic parameters ( $\varepsilon_{\text {reff }}, \alpha, d$, and $s$ ) of the final prototypes modeled by perforations, using triangular unit cells of holes, are also summarized in Table I, where the filling factor $(\alpha)$ is the fraction of area (or volume) of substrate material removed by the perforations to smoothly lower the permittivity from 7.1 to 2.9, depending on the diameter $(d)$ and distance $(s)$. The complete mathematical expressions to obtain the set of the characteristic parameters, which define the perforated lens, can be found in [12]. 


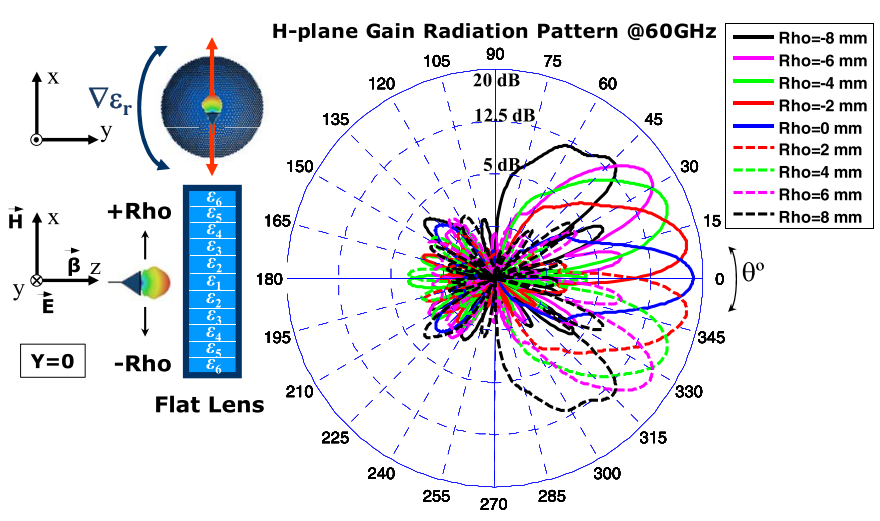

Fig. 3. H-plane gain radiation pattern simulation results at $60 \mathrm{GHz}$ for each Rho position of the WR-15 along $x$-dimension of the circular LTCC lens.

\section{Simulation Results}

In this section, the two designed dielectric flat lenses are briefly numerically analyzed to test their focusing capabilities and performance in the whole frequency band of interest.

1) Circular LTCC Flat Lens Simulation Results: The circular perforated flat lens model has been simulated at $60 \mathrm{GHz}$ band, from 57 to $66 \mathrm{GHz}$, using the time-domain solver of CST Microwave Studio. A complete set of nine different simulations have been performed corresponding to different discrete positions of a radiating element (which could correspond to the positions of antenna elements in a switched-beam array) along $x$-direction (see Fig. 3), going from Rho $=-8 \mathrm{~mm}$ to Rho $=+8 \mathrm{~mm}$, in steps of $2 \mathrm{~mm}$, testing the gain performance and beam-steering capabilities of the lens. The radiating element used consists of a rectangular aperture, a WR-15 openended waveguide model, with the $E$-field linearly polarized along the $y$-direction, which provides an efficient illumination of the lens with around $-14 \mathrm{~dB}$ edge taper in the $\mathrm{H}$-plane. The WR15 model is well matched $\left(S_{11}<-10 \mathrm{~dB}\right)$ in the whole frequency band and for all the feeding positions. The WR-15 open-ended waveguide has been chosen to feed the lenses during the simulations and measurements because it represents a standard very well-known topology for antennas, instead of using other antenna alternatives, despite this would lead to a volumetric antenna configuration. However, a completely planar antenna architecture suitable for mobile devices can be achieved, for example, with the lenses illuminated by a planar array of CPW-fed slot antennas, instead of an openended waveguide, as it is demonstrated in [9]. Nevertheless, in [9] it is shown that the lens performance in terms of gain radiation patterns is comparable to the performance achieved when the lens are fed by an open-ended waveguide. Moreover, the WR-15 feeding offers more flexibility in the setup during the experimental part of this paper. Then, for each Rho position of the feeding waveguide, the corresponding H-plane radiation patters are plotted at $60 \mathrm{GHz}$ in Fig. 3. The simulation results at $60 \mathrm{GHz}$ indicate that with the proposed design we are able to achieve up to $18.6 \mathrm{~dB}$ of broadside gain, beam-steering capabilities in both planes from $-25^{\circ}$ to $+25^{\circ}$ with around $17 \mathrm{~dB}$ gain, and up to $\pm 45^{\circ}$ with around $14 \mathrm{~dB}$ gain, with low sidelobe levels (SLLs). Note that given the lens symmetry

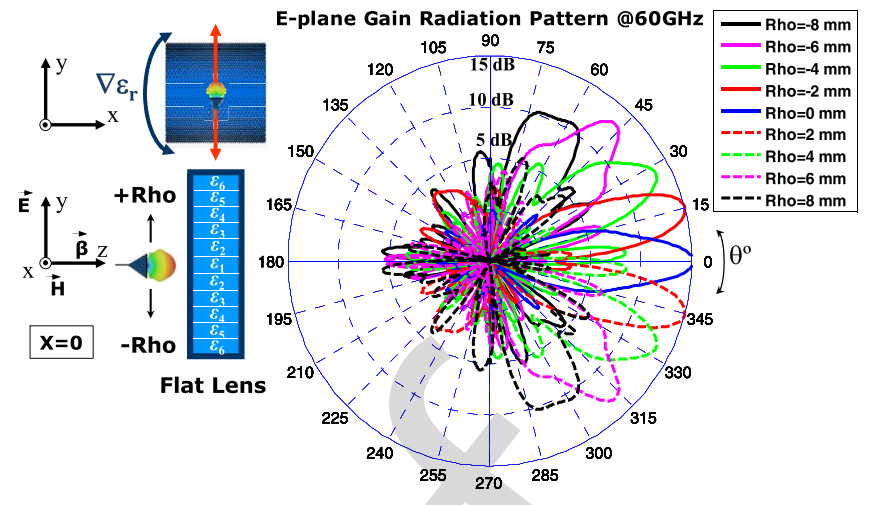

Fig. 4. E-plane gain radiation pattern simulation results at $60 \mathrm{GHz}$ for each Rho feeding position of the WR-15 waveguide along gradient axis of the lens.

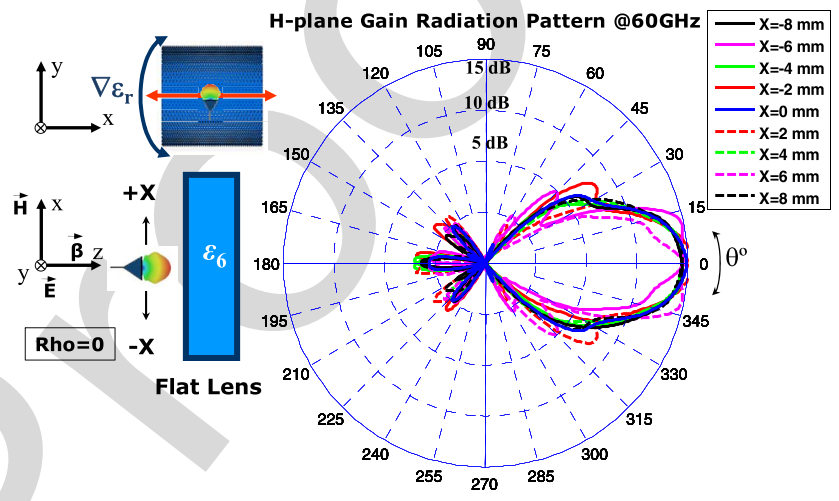

Fig. 5. H-plane gain radiation pattern simulation results at $60 \mathrm{GHz}$ for each $X$ feeding position of the WR-15 waveguide along constant axis of the lens.

identical E-plane radiation patterns are obtained when the lens is fed in the same way as for the H-plane, and therefore are not shown. Moreover, very good gain stability within the whole $60 \mathrm{GHz}$ band is observed from the simulated bandwidth performance, plotted in Fig. 6.

2) Cylindrical LTCC Flat Lens Simulation Results: The cylindrically distributed parameters perforated flat lens model has been simulated from 57 to $66 \mathrm{GHz}$, using the time-domain solver of CST Microwave Studio, in the same way as in the previous section, along the gradient-index axis (to test its beam-steering capabilities), and along the constant-index axis (to test that the beam produced by the lens remains almost invariant despite the position of the feeding antenna in this specific dimension). Therefore, a complete set of nine different simulations have been performed corresponding to different discrete positions of a radiating element along the gradient-index axis (i.e., $y$-direction in Fig. 2), going from Rho $=-8 \mathrm{~mm}$ to Rho $=+8 \mathrm{~mm}$, in steps of $2 \mathrm{~mm}$, testing the beam-steering capabilities of the lens. Another set of nine different simulations have also been performed moving the radiating element along the constant-index axis (i.e., $x$-direction), to test that the beam produced by the lens remains almost invariant despite the position of the feeding antenna.

In both sets of simulations, the radiating element used is a rectangular aperture model, a WR15 waveguide $\left(S_{11}<-10 \mathrm{~dB}\right)$ in the whole frequency band), which provides 


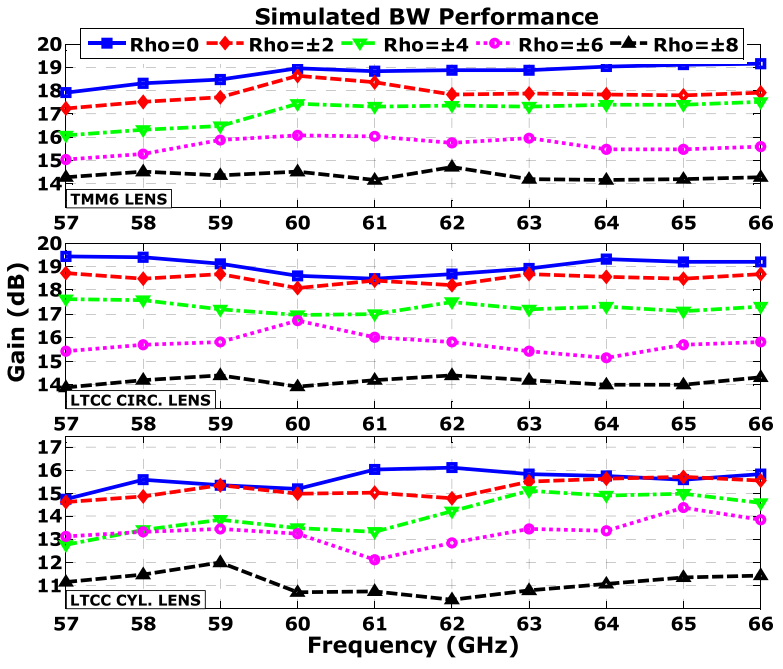

Fig. 6. Simulated bandwith performance: gain for different Rho positions of the WR15 feeding the designed lenses in the whole frequency band of interest. an efficient lens illumination with around $-12 \mathrm{~dB}$ edge taper in the E-plane. Then, for each position of the radiating waveguide, the corresponding E-plane and $\mathrm{H}$-plane radiation patterns are plotted at $60 \mathrm{GHz}$ in Figs. 4 and 5, for the gradient-index and constant-index cases, respectively. As it is shown, the expected behavior of the lens is obtained for both described cases: a radiation beam with around $15 \mathrm{~dB}$ of gain can be steered $\pm 15^{\circ}$ in the gradient axis, and up to $\pm 60^{\circ}$ with more than $10 \mathrm{~dB}$ gain, while a radiation beam with around $15 \mathrm{~dB}$ gain is practically maintained invariant pointing to the broadside direction despite the feeding aperture is being moved along the constant-index axis, allowing us to perform the beam scanning in this direction by using a different technique. The maximum gain obtained in our numerical results is slightly lower compared to the gain achieved with the inhomogeneous circular lenses, because in this case the cylindrically distributed parameters flat lens is performing the phase correction only in one single dimension instead of two. For this reason, the radiation beam obtained is a fan-beam type pattern (i.e., a beam with a narrow beamwidth in one dimension, broader in the orthogonal), which could be also very interesting for some particular applications such as radar and imaging systems, and more specifically for high-speed indoor communication systems at $60 \mathrm{GHz}$, in which this kind of pattern has been successfully assessed [17]. From the simulation results, we also obtain total and radiation efficiencies around $90 \%-95 \%$ for the lens fed with the aforementioned rectangular aperture, since a low-loss LTCC substrate is used.

3) Rogers TMM6 Flat Lens Simulation Results: For comparison purposes, the circular dielectric flat lens introduced in [11] is also considered during the experimental assessments carried out along this paper. Since the radiation pattern numerical results obtained for this lens have been already published in [11], they are not shown here. Instead, the bandwidth performance for the circular TMM6 lens, and for the two new LTCC lenses, is plotted in Fig. 6.

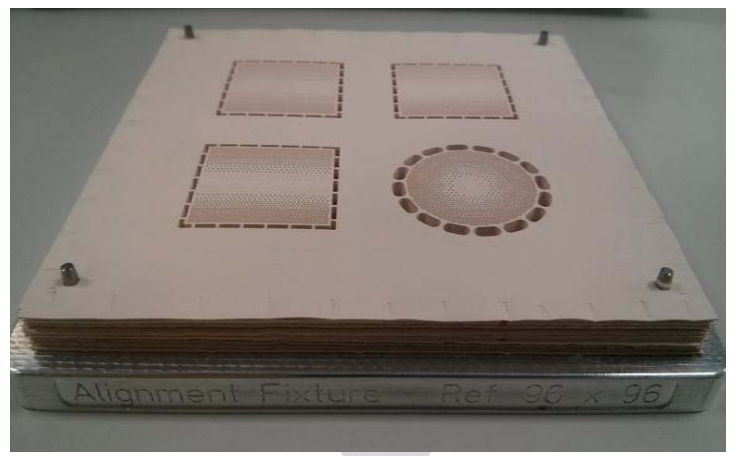

Fig. 7. LTCC dielectric flat lens prototypes fabrication: 31 DuPont 9k7 layers aligned and stacked together before the lamination process.

\section{LTCC FABRICATION OF THE PROTOTYPES}

Once the new designed LTCC lenses have been numerically tested, and promising simulation results were obtained, different prototypes have been fabricated at the facilities of the Universitat Politècnica de València in LTCC technology in order to, first, characterize their performance with a complete set of measurements, and then, experimentally evaluate their practical application as smart antenna solution for high data rate $5 \mathrm{G}$ millimeter-wave systems. A good description of the complete LTCC fabrication process can be found in [19]. Essentially, the LTCC process consists in building a multilayered substrate structure with the capability of printing different metallization individually in each single dielectric glass/ceramic sheet (called green tape). Thus, LTCC allows processing all the design layers separately.

Once all the layers are processed in parallel, separately, they are stacked, laminated together at high pressure in an isostatic process (around $210 \mathrm{~kg} / \mathrm{cm}^{2}$ ), and co-fired (sintering process) at a temperature of $850{ }^{\circ} \mathrm{C}$ during $26.5 \mathrm{~h}$. After a preconditioning process, in which each sheet of smooth green-tape dielectric substrate is heated up to $120{ }^{\circ} \mathrm{C}$ during $20 \mathrm{~min}$, we perform at each layer a total of around 1500 holes with a via punching process machine, to finally achieve the desired gradient-index permittivity profile in one axis, while a constant-index profile is achieved in the orthogonal one, for the cylindrically distributed lens, and a gradient index along both axis, for the circular lens. These small holes, of only 0.4 and $0.2 \mathrm{~mm}$ in diameter, are performed on the soft $254 \mu \mathrm{m}$ thickness DuPont GreenTape 9k7 dielectric substrate. After the punching process, the 31 layers needed to finally build the lens are stacked together, laminated, and sintered in order to obtain a single monolithic structure of $7 \mathrm{~mm}$ thickness. During the lamination and sintering LTCC processes, the material is shrinking $11.8 \%$ in $z$-direction and $9.1 \%$ in $x$ - and $y$-directions, and therefore, we previously considered this shrinkage of the substrate material before manufacturing the final lens design to achieve the characteristic parameters explained in Section II (lens thickness, via-hole dimensions, and separation between holes).

It is remarkable that the proposed fabrication method reduces considerably the final fabrication time compared to the fabrication time needed for manufacturing the TMM6 lens 


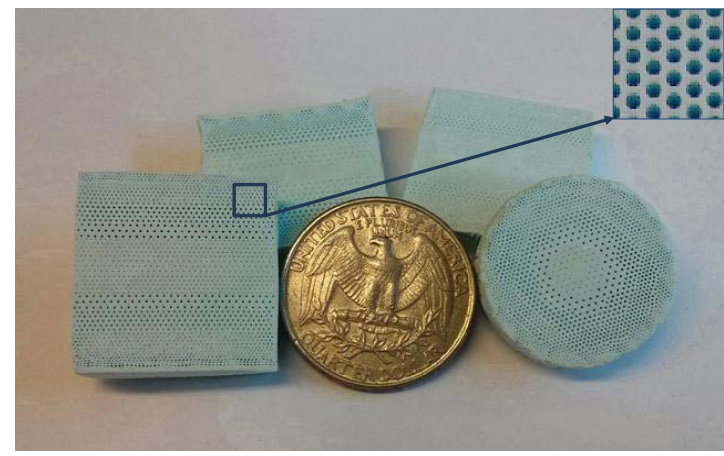

Fig. 8. LTCC dielectric flat lens prototypes with the effective permittivity circularly and cylindrically distributed. A microscopic image of a high hole density zone is shown in the inset of the upper-right corner.

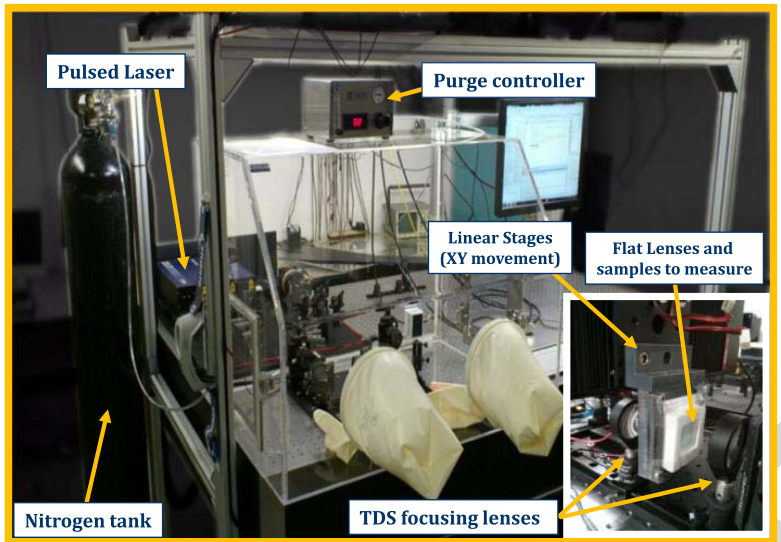

Fig. 9. TDS system placed on an optical talbe used to characterize different materials. A detailed image of the two focusing lenses of the system and the lens under test placed in between is shown in the inset.

introduced in [11], which was huge using carbide drills on a hard substrate, because the LTCC process allows to perform 1000 holes/min on each soft substrate layer. A photograph of the set of 31 DuPont LTCC material layers stacked to build the lenses is shown in Fig. 7, and a photograph of final prototypes is shown in Fig. 8, where a detailed microscopic image of a high-density zone of holes is additionally provided.

\section{Flat Lenses Measurement Results}

A set of measurements have been conducted at AntennaLab facilities of the Universitat Politècnica de Catalunya in order to characterize the performance of the introduced flat lenses for future high-speed 5G millimeter-wave applications.

\section{A. Flat Lenses Permittivity Profile Measurements}

Before testing the performance of the two dielectric flat lenses in terms of radiation patterns parameters, S-parameters, or efficiencies, it is fundamental and very interesting to assert that the required permittivity profiles have been achieved after the LTCC fabrication process.

With the described purpose, to precisely measure the permittivity profile of the fabricated prototypes a time-domain spectroscopy (TDS) system has been used. Our complete TDS measurement system is shown in Fig. 9. It consists of a femtosecond pulsed laser, which generates very short pulses that are sampled by using an optical delay stage. Once the complete

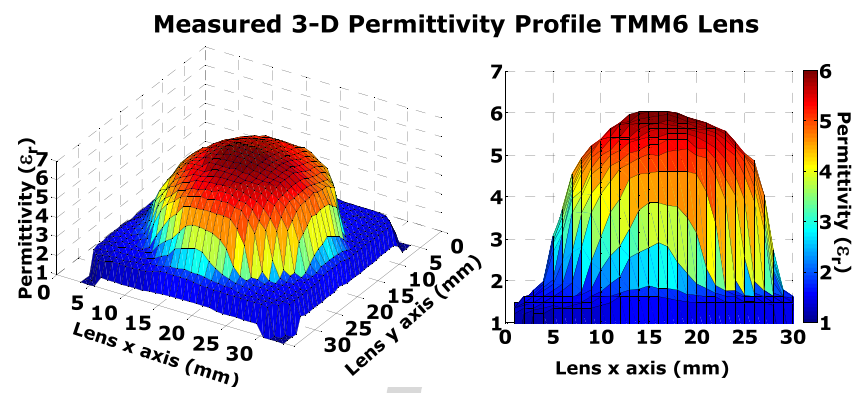

Measured 3-D Permittivity Profile LTCC Circular Lens

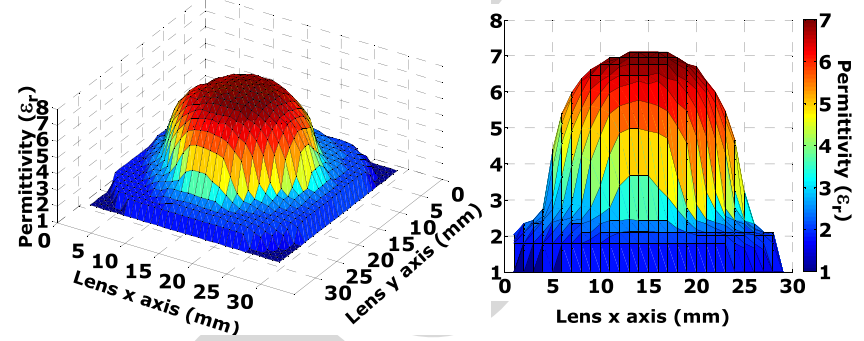

Measured 3-D Permittivity Profile LTCC Cylindrical Lens

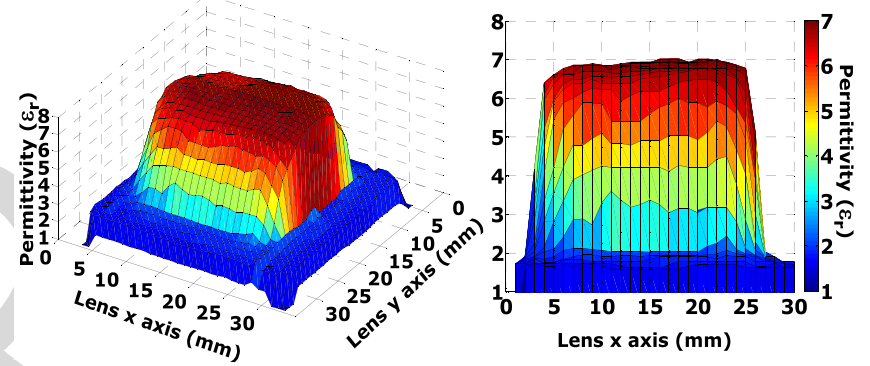

Fig. 10. 3-D representation of the mesured permittivity profile for the circular TMM6 lens (top), LTCC circular (middle), and LTCC cylindrical (bottom).

pulse is retrieved, a discrete Fourier transform is performed in order to obtain the spectrum, as it is usually realized in most of the TDS systems. In this specific case, despite our TDS system is a terahertz-TDS system, which is able to measure up to $1-1.5 \mathrm{THz}$, it is also capable of measuring with a dynamic range (DR) above $30 \mathrm{~dB}$ around $60 \mathrm{GHz}$, and with a DR above $50 \mathrm{~dB}$ around $100 \mathrm{GHz}$. Taking advantage of the small beam spot generated by our TDS system, which is collimated with two focusing lenses placed after the photoconductive receiver and transmitter antennas, we are able to precisely characterize the permittivity of different materials by using the delay produced introducing the sample in between, compared to the signal in free space. First, a solid sample of the DuPont 9k7 LTCC material has been measured, validating the maximum permittivity around 7 , as it was expected. After that, some different samples with uniform hole distribution have also been tested, obtaining the expected results as well, confirming the anticipated behavior.

Therefore, in order to measure the complete permittivity profile over the whole flat lens surface, the prototype is placed in between the two focusing lenses of the TDS system. With the help of two linear stages (to perform the specific movement needed in the $x$ - and $y$-axes), the TDS narrow radiation beam is scanned in steps of $1 \mathrm{~mm}\left(\lambda_{060 \mathrm{GHz}} / 5\right)$ over the lens surface. 


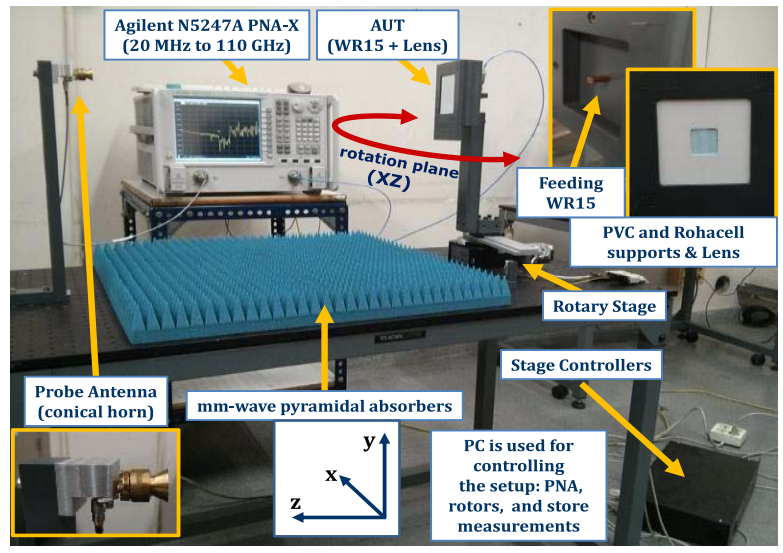

Fig. 11. Far-field radiation pattern measurement setup at $60 \mathrm{GHz}$ band. Detailed images of the WR-15 and lens on PVC supports are shown in the insets.

The 3-D representations of the measured permittivity profiles for the circular TMM6, and for the circular and cylindrical LTCC lenses, are plotted in Fig. 10. As it is shown, despite the physical shape of the designed lenses, with an absolutely planar structure, the permittivity profile is very well defined in all the cases for all the considered lenses, thus demonstrating the good fabrication results, confirming the viability in LTCC fabrication process.

\section{B. Flat Lenses Performance Evaluation}

A complete set of electromagnetic performance measurements for all the designed flat lenses has been carried out in the AntennaLab facilities of the UPC.

1) Radiation Pattern Measurement Results: The far-field radiation patterns produced by all the considered lenses fed with a WR-15 open-ended waveguide have been measured from 57 to $66 \mathrm{GHz}$ using the measurement setup shown in Fig. 11. It is composed of an Agilent N5247A vector network analyzer, a precision rotary stage to perform the scanning of the antennas under test (AUT) in the $x z$ plane (see Fig. 11), stage controllers, a WR-15 waveguide to feed the lens, a conical horn antenna used as a probe, some RF absorbers in order to avoid undesired reflections between the instrumentations, and a computer for controlling the automatization of the complete setup.

A total of nine measurements have been performed for the circular LTCC lens corresponding to different Rho feeding positions of the transmitting WR-15 waveguide (going from Rho $=-8 \mathrm{~mm}$ to Rho $=+8 \mathrm{~mm}$ ) in steps of $2 \mathrm{~mm}$ along the $x$-direction, with the waveguide linearly polarized in the $y$-direction, as it is depicted in the scheme of Fig. 12. Once the radiation patterns are measured, in order to obtain the gain radiation patterns, the AUT is replaced for a wellknown conical horn antenna (used as a reference) to perform a power level comparison. Therefore, the corresponding H-plane gain radiation pattern results are plotted in Fig. 12 at $60 \mathrm{GHz}$. In general, very good agreement is observed between simulation results (Fig. 3) and measurements. In the broadside direction we achieve up to $17.5 \mathrm{~dB}$ gain, with

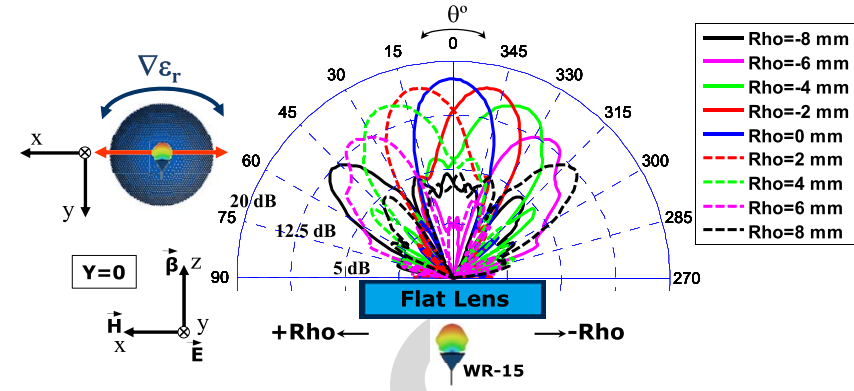

Fig. 12. Complete set of measured $\mathrm{H}$-plane gain radiation patterns at $60 \mathrm{GHz}$ for each Rho feeding position of the WR-15, for the circular LTCC lens.

TABLE II

SUMMARY OF TMM6 AND LTCC CIRCULAR LENSES PERFORMANCE AT $60 \mathrm{GHz}$ (H-Plane PARAMETERS)

\begin{tabular}{|c|c|c|c|c|c|c|c|c|}
\hline \multirow{2}{*}{ Rho } & \multicolumn{4}{|c|}{ TMM6 Circular Lens } & \multicolumn{4}{c|}{ LTCC Circular Lens } \\
\cline { 2 - 9 } & Gain & $\left(\boldsymbol{\theta}^{\circ}\right) \mathbf{s c a n}$ & $\boldsymbol{\Delta} \boldsymbol{\theta}_{-\mathbf{3 B}}$ & $\mathbf{S L L}$ & Gain & $\left(\boldsymbol{\theta}^{\circ}\right) \mathbf{s c a n}$ & $\boldsymbol{\Delta} \boldsymbol{\theta}_{-\mathbf{3 d B}}$ & SLL \\
\hline $0 \mathrm{~mm}$ & $18.3 \mathrm{~dB}$ & $0^{\circ}$ & $14^{\circ}$ & $-18 \mathrm{~dB}$ & $17.5 \mathrm{~dB}$ & $0^{\circ}$ & $21^{\circ}$ & $-15.8 \mathrm{~dB}$ \\
\hline $\pm 2 \mathrm{~mm}$ & $17.2 \mathrm{~dB}$ & $\pm 10^{\circ}$ & $15.1^{\circ}$ & $-13 \mathrm{~dB}$ & $16.7 \mathrm{~dB}$ & $\pm 12^{\circ}$ & $22^{\circ}$ & $-12 \mathrm{~dB}$ \\
\hline $\pm 4 \mathrm{~mm}$ & $16.6 \mathrm{~dB}$ & $\pm 22^{\circ}$ & $16.7^{\circ}$ & $-11.2 \mathrm{~dB}$ & $15.1 \mathrm{~dB}$ & $\pm 23^{\circ}$ & $23^{\circ}$ & $-8.9 \mathrm{~dB}$ \\
\hline $\pm 6 \mathrm{~mm}$ & $14.7 \mathrm{~dB}$ & $\pm 32^{\circ}$ & $17.8^{\circ}$ & $-10.5 \mathrm{~dB}$ & $12.9 \mathrm{~dB}$ & $\pm 37^{\circ}$ & $20^{\circ}$ & $-12.2 \mathrm{~dB}$ \\
\hline $\pm 8 \mathrm{~mm}$ & $13.7 \mathrm{~dB}$ & $\pm 48^{\circ}$ & $21^{\circ}$ & $-7.8 \mathrm{~dB}$ & $11.2 \mathrm{~dB}$ & $\pm 48^{\circ}$ & $17^{\circ}$ & $-7.8 \mathrm{~dB}$ \\
\hline
\end{tabular}

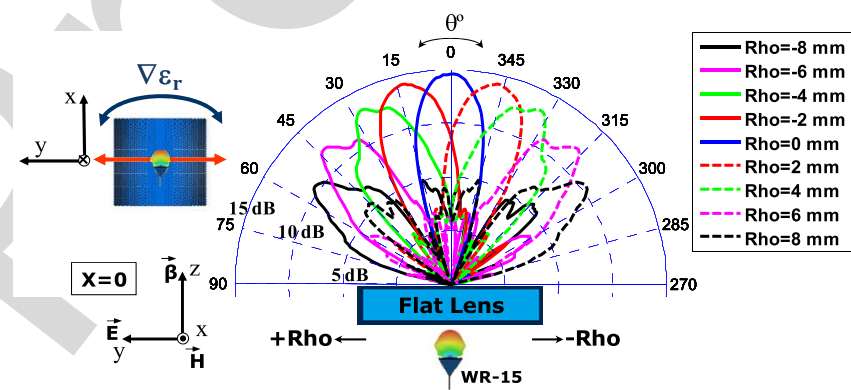

Fig. 13. Measured E-plane gain radiation patterns at $60 \mathrm{GHz}$ for each Rho feeding position of the WR-15 waveguide along gradient axis of the lens.

beam-steering capabilities from $-25^{\circ}$ to $+25^{\circ}$ with around $15 \mathrm{~dB}$, and up to $\pm 45^{\circ}$ with more than $11 \mathrm{~dB}$ gain. Additionally, the most important radiation pattern parameters at $60 \mathrm{GHz}$ are summarized in Table II, in order to concisely compact all the interesting and most relevant measurement results for a better analysis in the next experimental section in which the practical use of the three considered lenses as smart antenna systems is evaluated.

In the same way, a total of nine measurements have been performed for the cylindrical LTCC flat along the gradientindex axis of the lens, and nine additional measurements along the constant-index axis, in order to obtain the gain radiation patterns produced by the lens when is fed by a WR-15 waveguide. Therefore, the corresponding E-plane gain radiation patterns results are plotted in Fig. 13 at $60 \mathrm{GHz}$ (WR-15 with the electric field $y$-direction polarized, as it is depicted).

As it is observed, as the Rho feeding position is moved leftwards, the high-gain radiation pattern produced by the lens is steered rightwards (and vice versa), accordingly. Compared to the simulation results (Fig. 4), in general there is a very good agreement. $\mathrm{Up}$ to $14.8 \mathrm{~dB}$ gain in the broadside 


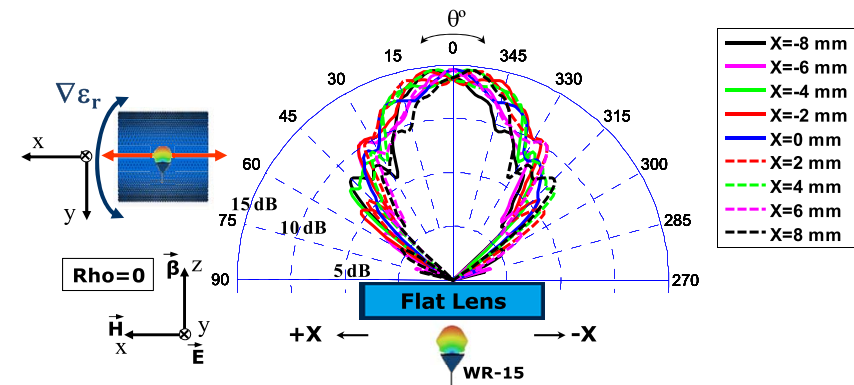

Fig. 14. Measured H-plane gain radiation patterns at $60 \mathrm{GHz}$ for each $X$ feeding position of the WR-15 waveguide along constant axis of the lens.

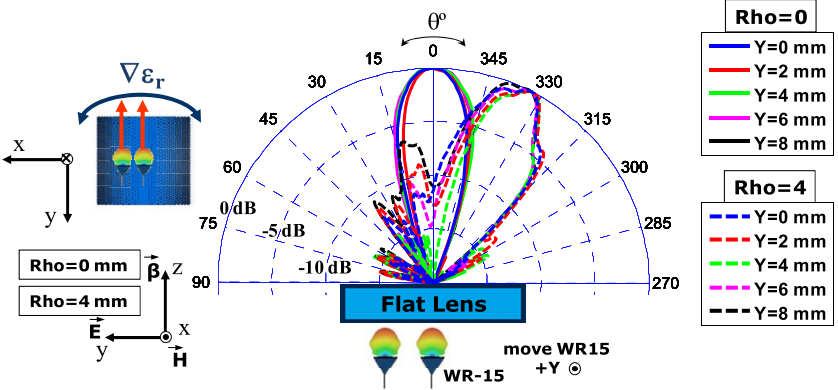

Fig. 15. Measured normalized E-plane radiation patterns at $60 \mathrm{GHz}$ (cut along gradient-index axis) moving the WR-15 in the constant axis of the lens (along $X$ ) for Rho $=0 \mathrm{~mm}$ and Rho $=4 \mathrm{~mm}$ feeding positions.

direction is achieved, with around $13 \mathrm{~dB}$ at $\pm 25^{\circ}$, and more than $10 \mathrm{~dB}$ when the beam is scanned $\pm 55^{\circ}$. In addition, the corresponding nine H-plane gain radiation patterns for nine different positions of the WR-15 waveguide along the constant-index axis of the lens (i.e., $x$-direction), maintaining the Rho position centered to the lens (Rho $=0 \mathrm{~mm}$ ), are plotted in Fig. 14. As it is shown, despite moving the feeding aperture, the beams are maintained almost invariant in this specific dimension, as it was expected.

Moreover, going one step further in this sense, the E-plane radiation patterns corresponding at different $X$ feeding positions of the WR-15 along the constant-index axis of the lens, keeping invariant the Rho position, are plotted in Fig. 15. Only the Rho $=0 \mathrm{~mm}(X=0-8 \mathrm{~mm})$ and Rho $=4 \mathrm{~mm}$ $(X=0-8 \mathrm{~mm})$ feeding positions are plotted in order to avoid cluttering the figure, but it is enough to observe and confirm the previously described behavior, which is also obtained for the rest of the feeding positions.

As it is noticed, although the WR-15 is moved in the $x$-dimension, the E-plane (vertical cut, $y$-direction in Fig. 15), is maintained practically invariant for all $X$ positions of the open-ended waveguide. As for the circular LTCC lens, the most important radiation pattern parameters at $60 \mathrm{GHz}$ are summarized in Table II for the cylindrical LTCC lens. Moreover, the same set of radiation patterns measurements are also carried out for the TMM6 lens introduced in [11] with our setup. Then, for comparison purposes and due to the fact that this lens is also evaluated in the next experimental section, the most important radiation pattern parameters at $60 \mathrm{GHz}$ are also summarized in Table II. As it is shown, with the circular LTCC lens, we achieve similar radiation pattern

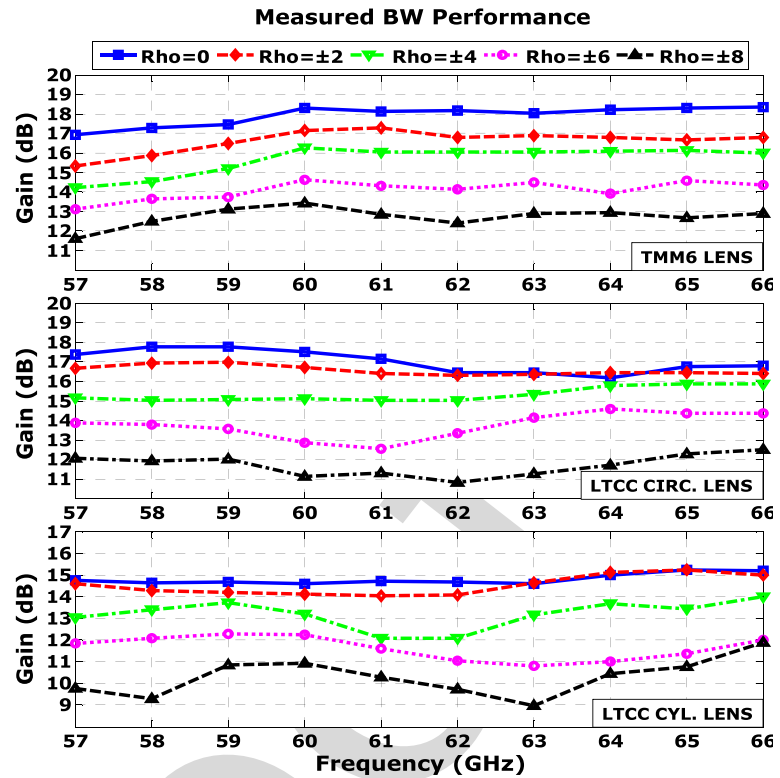

Fig. 16. Measured bandwidth performance: maximum gain for different Rho feeding positions of the WR-15 along gradient-index axis of the lenses.

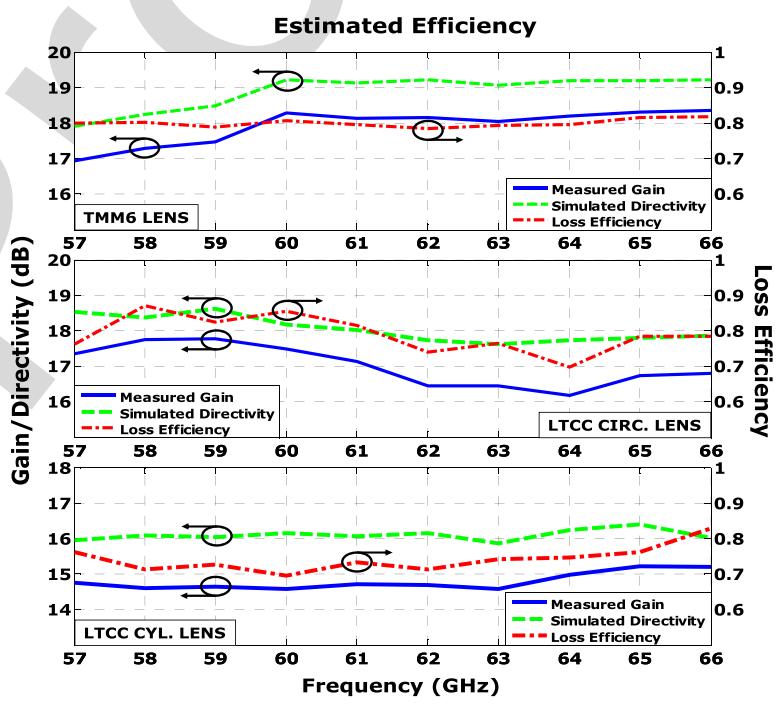

Fig. 17. Estimated loss efficiency computed from simulated directivity and measured gain values for the three considered lenses.

characteristics as with the circular TMM6 lens, with slightly lower gain values at some frequencies and scanning angles, because in this case the highest permittivity value is 7 instead of 6 , thus slightly higher reflection is obtained in the dielectricvacuum (free space) transition.

2) Measured Bandwidth Performance: In addition, the gain over the whole $60 \mathrm{GHz}$ frequency band of interest has been measured for all the Rho feeding positions, and it is plotted in Fig. 16, for the three considered dielectric flat lenses. As it shown, very good gain stability is observed for the three lenses, confirming the good broadband behavior obtained in the numerical results. This is a remarkable result because in general it is very difficult to achieve antenna systems with broadband operation behavior.

3) Estimated Efficiencies: The estimated loss efficiencies for the three flat lenses are also reported in Fig. 17, 


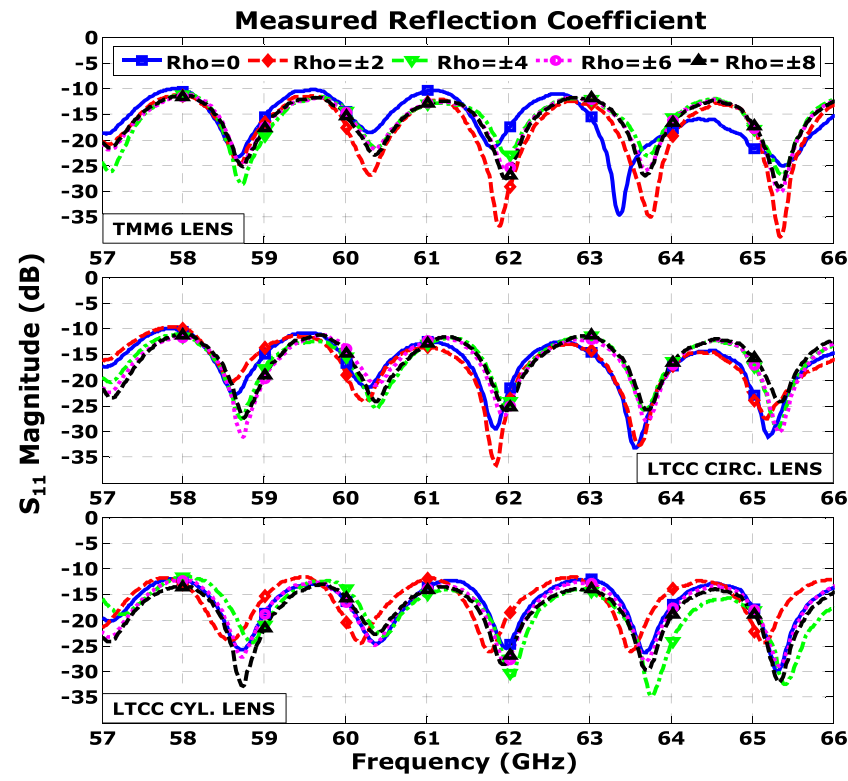

Fig. 18. Measured $S_{11}$ parameter of the three lenses for different Rho feeding positions of the WR-15 waveguide in the whole frequency band of interest.

from 57 to $66 \mathrm{GHz}$, computed from CST simulation results of the directivity and measured gain values, since with our setup we are not able to measure the complete 3-D radiation patterns in order to integrate the whole power to obtain directly the efficiency or the directivity. As it is shown, almost constant values around $70 \%-80 \%$, and above, are estimated in all the cases for the whole frequency band, since low-loss dielectric materials are used to build the lenses.

4) Measured Reflection Coefficient: The measured $S_{11}$ parameters obtained, after applying a short-open-load-thru (SOLT) calibration, for the different flat lenses fed with the corresponding WR-15 open-ended waveguide in the different Rho positions are plotted in Fig. 18, for the whole frequency band. As it is shown, all the measured reflection coefficients are below $-10 \mathrm{~dB}$, as it was expected.

\section{Assessment of the Flat Lens Performance in A REAL $60 \mathrm{GHZ}$ WPAN INDOOR ENVIRONMENT}

Once the three considered dielectric flat lenses have been fully electromagnetically characterized and remarkable good measurement results have been obtained, their performance is experimentally evaluated and compared to a single commercial omni-directional antenna, as well as their use as smart antennas is experimentally compared to a traditional ULA in real $60 \mathrm{GHz}$ WPAN environment.

\section{A. Introduction}

For this experimental part, we have considered an indoor scenario in the facilities of the Universidad Politécnica de Cartagena (UPCT) varying the position of the receiver (Rx) antenna. Three different positions for the $\mathrm{Rx}$ antenna have been measured forming an angle of $0^{\circ}, 22.5^{\circ}$, and $45^{\circ}$ with respect to the transmitting (Tx) antenna, which is placed in a fixed position. The receiver antenna is, in all the cases, a single commercial Q-par QOM55-65 VRA 55 to $65 \mathrm{GHz}$

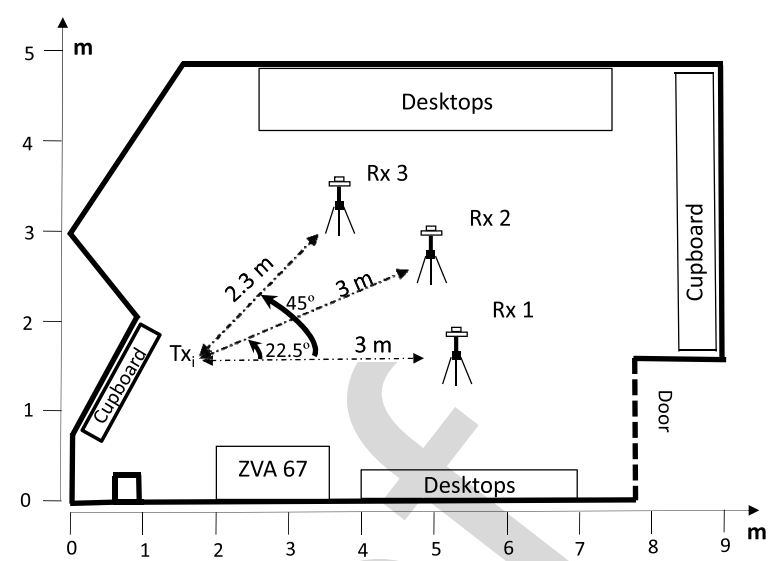

Fig. 19. Indoor scenario and experimental measurement setup arrangement.

omni-directional V-type antenna. The gain of this antenna varies from 4.3 to $5.2 \mathrm{~dB}$ within the considered 57 to $64 \mathrm{GHz}$ frequency band, and the typical $3 \mathrm{~dB}$ elevation beamwidth ranges from $24^{\circ}$ to $33^{\circ}$, while being omni directional in the horizontal plane. In this paper, the considered Tx antennas are the three presented lenses fed by the same rectangular aperture WR-15 waveguide used during the previous sections, the same commercial omni-directional antenna used in the Rx part, and a virtual ULA modeled with ten positions of this same omnidirectional antenna. The performance test with the considered Tx antennas is carried out in direct LOS conditions for all the angles between Tx and Rx, and also in OLOS conditions for the $0^{\circ}$ case. In the following sections, all the important considerations about the experimental scenario, channel sounder, and methodology are conveniently described before to proceed with the analysis of the measurement results.

\section{B. Experimental Scenario}

The scenario for this experimental study is a laboratory of the UPCT. The laboratory is an almost rectangular room of about $5 \mathrm{~m} \times 9 \mathrm{~m}$ furnished with several closets, desktops, and shelves. The laboratory scheme with the measurement setup arrangement is depicted in Fig. 19. As it is shown in Fig. 19, the three different considered Tx-Rx situations are established as follows: $3 \mathrm{~m}$ between $\mathrm{Tx}$ and $\mathrm{Rx}$ forming an angle of $0^{\circ}$ (first position), $3 \mathrm{~m}$ between $\mathrm{Tx}$ and $\mathrm{Rx}$ forming an angle of $22.5^{\circ}$ (second position), and at $2.3 \mathrm{~m}$ between $\mathrm{Tx}$ and $\mathrm{Rx}$ forming an angle of $45^{\circ}$ (third position).

\section{Channel Sounder and Methodology}

The channel sounder and the methodology employed in this paper are exactly the same as the followed and exhaustively explained in [20]. A VNA is used to measure the transmission $\left(S_{21}\right)$ parameter in order to obtain in the frequency domain the complex transfer function of a wireless system. The frequency domain function measured $H(f)$ is acquired. Then, the relative received power $(P)$ is computed. This parameter, which is defined as the ratio between the transmitted and the received powers, is important for $5 \mathrm{G}$ communication systems because describes the attenuation of the transmitted 


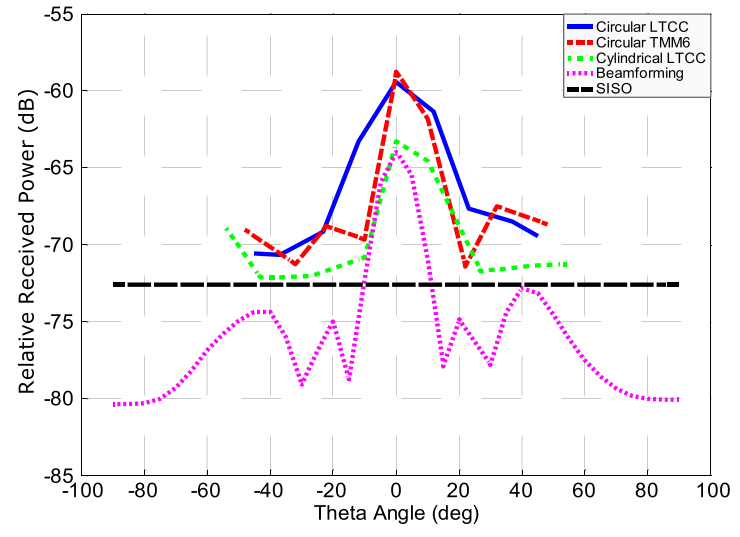

Fig. 20. Relative received power at first position $\left(\theta=0^{\circ}\right)$ in LOS conditions.

TABLE III

Summary of LTCC CYLINDRICAL LENS PERFormanCE AT $60 \mathrm{GHz}$ (E-Plane AND H-Plane PARAMETERS)

\begin{tabular}{|c|c|c|c|c|c|c|c|c|}
\hline \multirow{2}{*}{ Rho } & \multicolumn{3}{|c|}{ E-PLANE (along gradient axis) } & \multicolumn{4}{c|}{ H-PLANE (along constant axis) } \\
\cline { 2 - 9 } & Gain & $\left(\boldsymbol{\theta}^{\circ}\right)$ scan & $\boldsymbol{\Delta} \boldsymbol{\theta}_{-3 \mathrm{~dB}}$ & SLL & Gain & $\left.\boldsymbol{\theta}^{\circ}\right)$ scan & $\boldsymbol{\Delta}_{-3 \mathrm{~dB}}$ & SLL \\
\hline $0 \mathrm{~mm}$ & $14.6 \mathrm{~dB}$ & $0^{\circ}$ & $19^{\circ}$ & $-17.7 \mathrm{~dB}$ & $14.6 \mathrm{~dB}$ & $0^{\circ}$ & $48^{\circ}$ & $-17.5 \mathrm{~dB}$ \\
\hline $\pm 2 \mathrm{~mm}$ & $14.1 \mathrm{~dB}$ & $\pm 13^{\circ}$ & $21^{\circ}$ & $-12 \mathrm{~dB}$ & $14.4 \mathrm{~dB}$ & $0^{\circ}$ & $44^{\circ}$ & $-8.9 \mathrm{~dB}$ \\
\hline $\pm 4 \mathrm{~mm}$ & $13.2 \mathrm{~dB}$ & $\pm 27^{\circ}$ & $20^{\circ}$ & $-11.5 \mathrm{~dB}$ & $14.3 \mathrm{~dB}$ & $0^{\circ}$ & $46^{\circ}$ & $-9.6 \mathrm{~dB}$ \\
\hline $\pm 6 \mathrm{~mm}$ & $12.3 \mathrm{~dB}$ & $\pm 43^{\circ}$ & $21^{\circ}$ & $-8.9 \mathrm{~dB}$ & $14.6 \mathrm{~dB}$ & $0^{\circ}$ & $35^{\circ}$ & $-10.6 \mathrm{~dB}$ \\
\hline $\pm 8 \mathrm{~mm}$ & $10.9 \mathrm{~dB}$ & $\pm 54^{\circ}$ & $17^{\circ}$ & $-5.5 \mathrm{~dB}$ & $14.5 \mathrm{~dB}$ & $0^{\circ}$ & $35^{\circ}$ & $-14 \mathrm{~dB}$ \\
\hline
\end{tabular}

radio link in a specific angular direction. Next, the time domain function $h(t)$ is obtained by using the inverse fast Fourier transform. Last, the power delay profile (PDP) and the RMSdelay spread $\left(\sigma_{\tau}\right)$, which represents the standard deviation of the PDP, are calculated, as it is exhaustively explained in [20]. In this case, the RMS delay spread is a fundamental parameter in order to have a notion of the multipath characteristics of a communications channel. The longer the RMS delay spread, the smaller the coherence bandwidth, which directly affects and limits the capacity in a $5 \mathrm{G}$ wireless communication system.

\section{Experimental Results}

In the following sections the experimental results obtained for the three considered positions are reported and compared for the three dielectric flat lenses, and for the ULA and SISO cases.

1) First Position Measurements: As it is depicted in Fig. 19, in the first position situation the Tx and the Rx antennas are separated $3 \mathrm{~m}$ forming an angle of $0^{\circ}$ between them. For the LOS condition, the absorbent panel placed in between the Tx and Rx is removed. Then, the methodology detailed in [20] is applied obtaining the following results for all the considered antennas.

For the LOS situation, the relative received power in function of the angle and the PDP are plotted for each different transmitting antenna in Figs. 20 and 21, respectively.

As it is observed in Fig. 20, the highest relative received power is achieved using the circular TMM6 as a transmitting antenna, as it was expected from the measured radiation pattern parameter results obtained in the previous sections (see Tables II and III). In any case, with all the designed

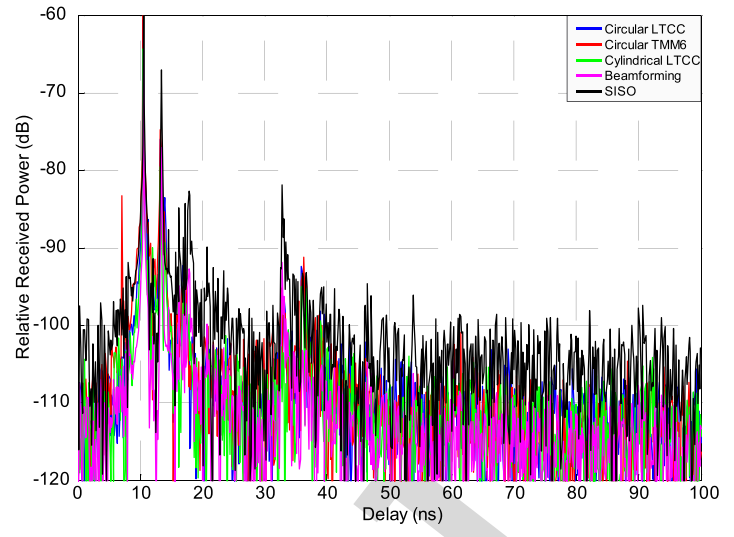

Fig. 21. PDP at first position $\left(\theta=0^{\circ}\right)$ in LOS conditions.

TABLE IV

Relative Received Power ANd RMS DELAy SPREAD VALUES FOR FIRST POSITION IN LOS CONDITIONS

\begin{tabular}{|c|c|c|}
\cline { 2 - 3 } \multicolumn{1}{c|}{} & Relative Received Power & RMS Delay Spread \\
\hline Circular LTCC & $-59.45 \mathrm{~dB}$ & $0.68 \mathrm{~ns}$ \\
\hline Circular TMM6 & $-58.79 \mathrm{~dB}$ & $0.66 \mathrm{~ns}$ \\
\hline Cylindrical LTCC & $-63.30 \mathrm{~dB}$ & $0.64 \mathrm{~ns}$ \\
\hline 10-elem. ULA & $-63.96 \mathrm{~dB}$ & $1.21 \mathrm{~ns}$ \\
\hline SISO & $-72.56 \mathrm{~dB}$ & $4.47 \mathrm{~ns}$ \\
\hline
\end{tabular}

lenses the relative received power is better compared to using a beamforming technique applied to the ten-element ULA. In Fig. 21, the measured PDP shows that for all cases, direct ray with highest power (LOS component) is received at $10.5 \mathrm{~ns}$. The rest of the components arrive attenuated in the next moments due to the multipath propagation. It is worthwhile mention that the shape obtained for all the PDPs is almost identical, which means that the situation of the antennas has been the same during the whole process of the measurements campaign, fact that is very difficult in this kind of measurements at these frequencies.

In the Table IV, the relative received power and the RMS delay spread calculated from the PDP for each evaluated transmitting antenna are summarized. As it is observed in Table IV, the highest relative received power is achieved with the circular TMM6 lens, which it has been stated before.

Additionally, the power difference among the rest is according to the measured gain values (see Tables II and III). For example, the measured gain difference obtained in previous sections between the circular TMM6 lens and the circular LTCC is around $0.8 \mathrm{~dB}$, which is almost the same relative received power difference obtained for this first measured position in LOS conditions. Similar results are also obtained comparing the TMM6 lens and the cylindrical LTCC lens: a measured gain difference of $3.7 \mathrm{~dB}$ between the two lenses, and a relative received power difference of $4.5 \mathrm{~dB}$. Moreover, a remarkable result is that the measured relative received power for the ten-element ULA is lower than the measured for all the designed lenses, being the SISO case the worst, and constant, independently of the angle, as it is shown in Fig. 22, since a single omni-directional antenna is used. 


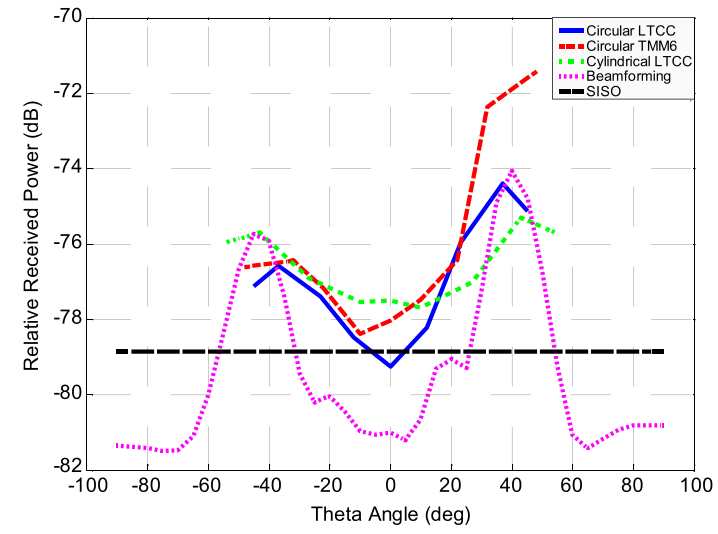

Fig. 22. Relative received power at first position $\left(\theta=0^{\circ}\right)$ in OLOS conditions.

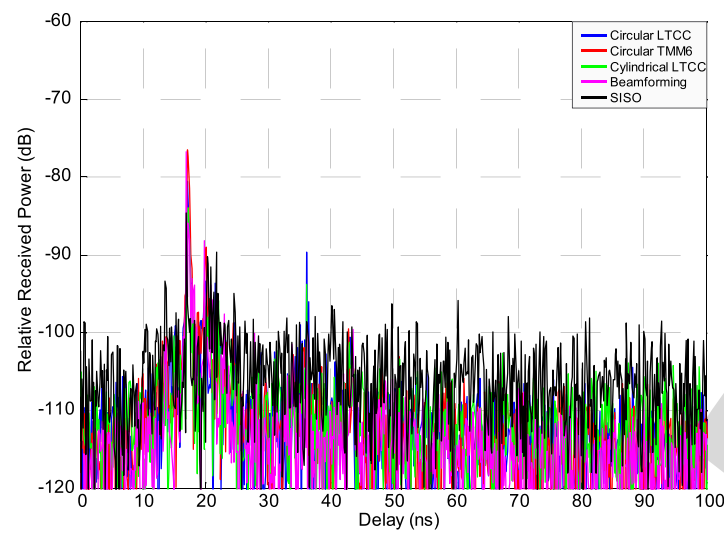

Fig. 23. PDP at first position $\left(\theta=0^{\circ}\right)$ in OLOS conditions.

Regarding the RMS delay spread, the computed values from measurements are very low for all the considered antennas due to the LOS situation, in which the signal is propagating without facing any obstacle. For the lens antennas, the results are very similar, below $1 \mathrm{~ns}$, being for the cylindrical LTCC lens the lowest. However, for the ULA case the RMS delay spread is the double due to the diversity, and for the SISO is even higher because the use of the omni-directional antenna, which has a wider $-3 \mathrm{~dB}$ beamwidth radiation pattern.

For the same setup of the first measured position, we placed an absorbent panel in the middle of the Tx and Rx antennas. Therefore, in this case the direct ray is obstructed by the obstacle. In the same way as in the previous situation, the relative received power in function of the angle and the PDP is plotted for each transmitting antenna in Figs. 22 and 23, respectively. As it is shown in Fig. 23, the direct ray is canceled and a component with lower power than the previous one is received at $17.1 \mathrm{~ns}$. In Fig. 22, it is observed that at $0^{\circ}$, the received power is really low because this path is being obstructed for the absorbent panel. However, thanks to multipath propagation, around $40^{\circ}$ we are receiving a certain amount of power. For this angle, the TMM6 lens is still performing better than the rest of transmitting antennas, despite the ULA is steering the beam to the direction of maximum propagation, but it is clearly receiving less power.
TABLE V

Relative Received Power and RMS Delay SPREad Values FOR FIRST POSITION IN OLOS CONDITIONS

\begin{tabular}{|c|c|c|}
\cline { 2 - 3 } \multicolumn{1}{c|}{} & Relative Received Power & RMS Delay Spread \\
\hline Circular LTCC & $-74.38 \mathrm{~dB}$ & $18.81 \mathrm{~ns}$ \\
\hline Circular TMM6 & $-71.42 \mathrm{~dB}$ & $5.77 \mathrm{~ns}$ \\
\hline Cylindrical LTCC & $-75.29 \mathrm{~dB}$ & $36.01 \mathrm{~ns}$ \\
\hline 10-elem. ULA & $-74.05 \mathrm{~dB}$ & $42.78 \mathrm{~ns}$ \\
\hline SISO & $-78.83 \mathrm{~dB}$ & $33.18 \mathrm{~ns}$ \\
\hline
\end{tabular}

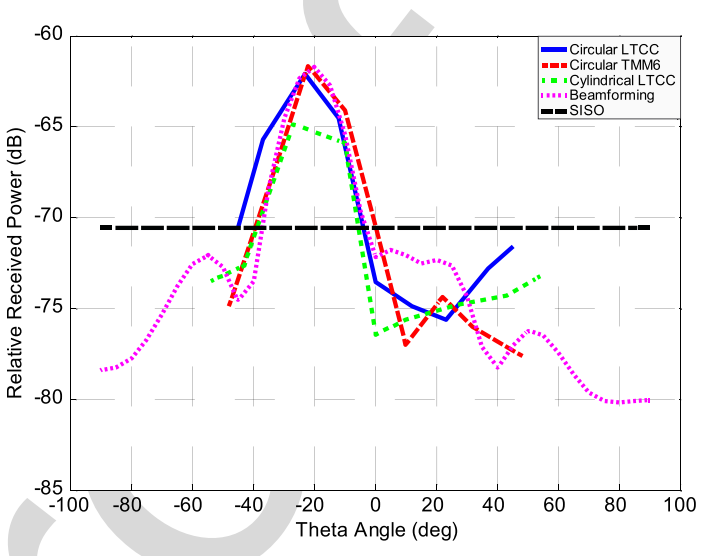

Fig. 24. Relative received power at second position $\left(\theta=22.5^{\circ}\right)$ in LOS conditions.

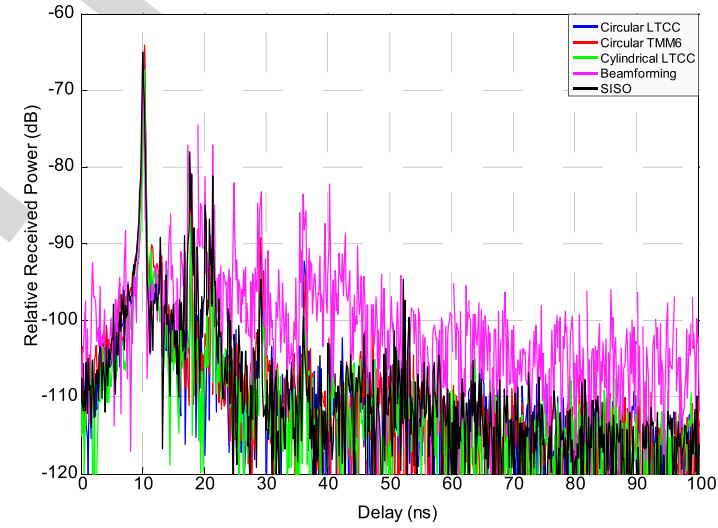

Fig. 25. PDP at second position $\left(\theta=22.5^{\circ}\right)$ in LOS conditions.

Table V shows a summary of the computed values for the relative received power and RMS delay spread for this OLOS situation in the first measurement position. Due to the obstacle, the received power decreases, while the RMS delay spread increases, as it was expected. It is observed that the lowest delay spread is also achieved with the TMM6 lens.

2) Second Position Measurements: As it is depicted in Fig. 19, in the second position situation the $\mathrm{Tx}$ and $\mathrm{Rx}$ antennas are separated $3 \mathrm{~m}$ forming an angle of $22.5^{\circ}$ between them in a LOS condition. In the same way as it has been previously described, the measurements are carried out. Therefore, the relative received power in function of the angle and the PDP are plotted for each different transmitting antenna in Figs. 24 and 25, respectively, and in the Table VI, the computed relative received power and the RMS delay spread are also summarized. 
TABLE VI

Relative Received Power and RMS DELAY SPREAD VALUES FOR SECOND POSITION IN LOS CONDITIONS

\begin{tabular}{|c|c|c|}
\cline { 2 - 3 } \multicolumn{1}{c|}{} & Relative Received Power & RMS Delay Spread \\
\hline Circular LTCC & $-62.11 \mathrm{~dB}$ & $1.84 \mathrm{~ns}$ \\
\hline Circular TMM6 & $-61.67 \mathrm{~dB}$ & $1.86 \mathrm{~ns}$ \\
\hline Cylindrical LTCC & $-64.87 \mathrm{~dB}$ & $1.38 \mathrm{~ns}$ \\
\hline 10-elem. ULA & $-61.75 \mathrm{~dB}$ & $12.37 \mathrm{~ns}$ \\
\hline SISO & $-70.54 \mathrm{~dB}$ & $4.11 \mathrm{~ns}$ \\
\hline
\end{tabular}

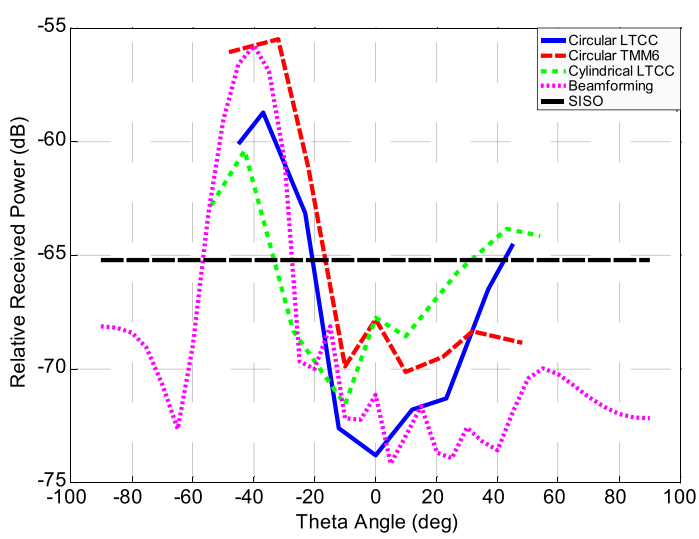

Fig. 26. Relative received power at third position $\left(\theta=45^{\circ}\right)$ in LOS conditions.

As it is shown in Fig. 25, the strongest component is received at $10.1 \mathrm{~ns}$, a similar time delay as for the first position in LOS situation, but the received power is slightly lower because the antennas are forming $22.5^{\circ}$ between them. In this case, the highest received power value is obtained using the TMM6 lens, and the lowest RMS delay spread is achieved with the cylindrical LTCC lens. The power received with the ten-element ULA is almost the same as with the TMM6 lens, however, the RMS delay spread is considerably higher, nearly seven times higher in comparison to TMM6 lens, and up to nine times compared to the value obtained using the cylindrical LTCC lens, which is a remarkable result because directly affects the coherence bandwidth, which in turn limits the capacity in a wireless transmission system.

3) Third Position Measurements: The third position considered in this experimental study is also depicted in Fig. 19, defining a distance of $2.3 \mathrm{~m}$ separating the Tx and Rx antennas and forming an angle of $45^{\circ}$ between them in LOS conditions. For this particular wide-angle case, the measured relative power and the computed PDP are plotted in Figs. 26 and 27, respectively. In addition, the maximum relative received power and the RMS delay spread values are summarized in Table VII for each evaluated Tx antenna. As it is observed, the direct ray with the strongest component (LOS condition) is received at $7.7 \mathrm{~ns}$ for all the considered antennas. The rest of the components arrive delayed due to the multipath propagation, all of them with different levels of attenuation depending on which antenna is used. The maximum received power is centered around $40^{\circ}$, as it is shown in Fig. 26. Once again, the highest received power is achieved with the circular TMM6 lens, despite the wide steering angle in which the

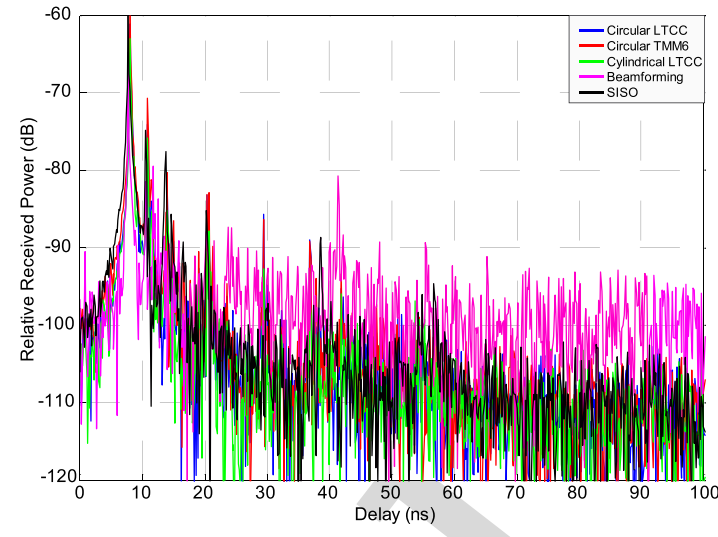

Fig. 27. PDP at third position $\left(\theta=45^{\circ}\right)$ in LOS conditions.

TABLE VII

Relative Received Power and RMS Delay SPREAd VALUES FOR THIRD POSITION IN LOS CONDITIONS

\begin{tabular}{|c|c|c|}
\cline { 2 - 3 } \multicolumn{1}{c|}{} & Relative Received Power & RMS Delay Spread \\
\hline Circular LTCC & $-58.75 \mathrm{~dB}$ & $1.80 \mathrm{~ns}$ \\
\hline Circular TMM6 & $-55.51 \mathrm{~dB}$ & $1.27 \mathrm{~ns}$ \\
\hline Cylindrical LTCC & $-60.37 \mathrm{~dB}$ & $1.20 \mathrm{~ns}$ \\
\hline 10-elem. ULA & $-55.80 \mathrm{~dB}$ & $18.16 \mathrm{~ns}$ \\
\hline SISO & $-65.21 \mathrm{~dB}$ & $2.92 \mathrm{~ns}$ \\
\hline
\end{tabular}

Rx antenna is placed with respect to the Tx. Regarding the RMS delay spread, the results confirm the previously obtained in other situations, being the cylindrical LTCC lens the best option in order to obtain the lowest value, with a RMS delay spread of $1.2 \mathrm{~ns}, 15$ times lower than the obtained with the ten-element ULA.

\section{Switched-Beam Antenna Based on LTCC Dielectric Flat LENSES AND FREQUENCY-SCANNED ARRAYS}

In the last section of this paper, the design of an innovative switched-beam antenna array concept for 5G millimeter-wave applications, based on a practical application of the cylindrically distributed parameters LTCC flat lens, is presented and completely evaluated.

\section{A. Introduction}

As it has been demonstrated, taking advantage of the cylindrical effective parameter distribution of the lens, the beam scanning can be performed in one plane by moving (or selecting) the position of a radiating single element along the gradient-index axis, whereas the beam can be maintained invariant in the other direction, in which the effective parameters are kept constant, despite changing the radiating element position in this particular axis. Therefore, in this way, the beam scanning can be achieved in the constant-index axis of the lens by means of a different technique, a FSSA [21], [22], which it is also introduced in this final paper section, in order to realize not only a 1-D beam scanning but a 2-D beam scanning of high-gain radiation beams, in a compact millimeter-wave 


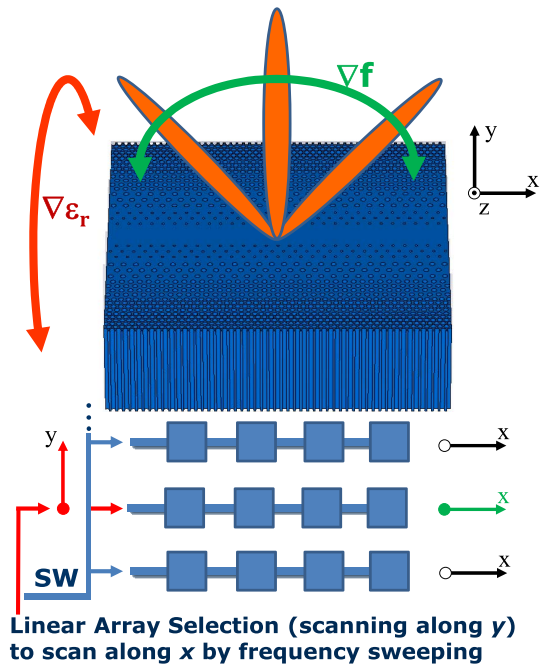

Fig. 28. Switched-beam antenna array concept with cylindrically distributed parameters flat lens and frequency-scanned array to perform beam-scanning in theta and phi, by frequency sweeping or selecting a specific linear array.

antenna solution, easy to integrate in a single monolithic structure with LTCC technology.

Therefore, the theoretical concept for the described behavior of the flat lens in front of a linear array of antennas distributed along the constant-index axis, which is able to scan its beam in this dimension by sweeping the frequency, is shown in Fig. 28.

\section{B. Frequency-Scanned Slot Antenna Array}

Considering that a broadside invariant radiation pattern is obtained in the constant-index axis of the flat lens, despite the feeding aperture is being moved along this axis, a linear frequency-scanned stripline-fed transverse slot antenna array with a particular structure has been designed to achieve beam scanning in one single plane by sweeping the frequency, taking advantage of the huge amount of available bandwidth for communication applications around $60 \mathrm{GHz}$.

1) Frequency-Scanned Slot Array Design and Geometry: In this kind of arrays, the beam-steering capability is obtained controlling the relative phase shift between the array elements by sweeping the operating frequency [21], instead of introducing phase delays by means of bulky and complex phase shifters, as it is common in traditional phased arrays.

The proposed linear array geometry is shown in Fig. 29. It consists of a set of ten transverse slots fed by a meandering stripline, which provides the required phase delay between slot elements in order to steer the beam when the frequency is conveniently changed. The signal is propagating through the stripline and it is coupling energy to each one of the slots, which in turn, is radiating the coupled energy to the free space. In this way, the slots which are closer to the stripline feeding point need to be less coupled than the slots which are far away from this point, because the signal is stronger at the beginning and tends to be smoothly weakened because it is being radiated at every consecutive slot it finds during its propagation. The stripline is terminated with a matched load in order to absorb the remaining power which is not being radiated after the last of the slots, thus avoiding undesired reflections. This array is

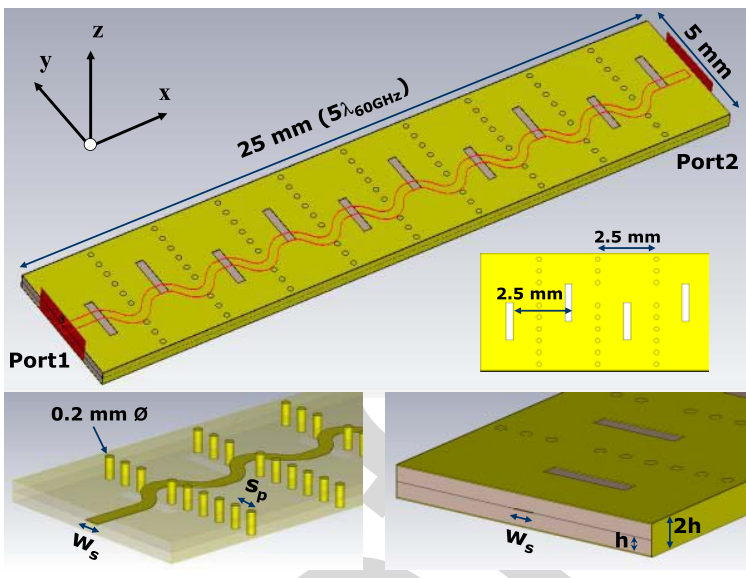

Fig. 29. Frequency-scanned stripline-fed transverse slot antenna array geometry: whole structure (top), and detailed images of the meandering stripline and the pin curtains (left) and two layer structure geometry dimensions (right).

a nonresonant structure, in which traveling waves are used for the excitation of the slots, opposed to resonant or standing wave arrays, in which a short circuit is placed at the end, instead of a matched load.

The total dimensions of this novel stripline-fed slot antenna array are $25 \mathrm{~mm} \times 5 \mathrm{~mm}\left(5 \lambda_{60 \mathrm{GHz}} \times 1 \lambda_{60} \mathrm{GHz}\right)$, with $508 \mu \mathrm{m}$ thickness. It is designed from two different Rogers Duroid $5880\left(\varepsilon_{r}=2.2 ; \tan (\delta)=0.004\right.$ at $60 \mathrm{GHz}$ [23] substrate layers of $254 \mu \mathrm{m}$ thickness. This substrate was chosen for its low losses and low permittivity values, which facilitate the radiation and improve the overall antenna efficiency.

The slot geometry plane is printed on the top substrate layer, while the meandering stripline and the ground-plane are printed on the bottom layer; thus the feeding line is placed in between top and bottom planes. The slot dimensions are all the same $(1.6 \mathrm{~mm} \times 0.3 \mathrm{~mm})$. The meandering stripline is designed in $370 \mu \mathrm{m}$ width, in order to ensure $50 \Omega$ at the feeding port.

As it is shown in Fig. 29, the ten slots are placed transversal to the feeding stripline, leaving a physical distance of $\lambda_{060 \mathrm{GHz}} / 2$ between them. The meandering stripline length is around $\lambda_{\mathrm{g} 60 \mathrm{GHz}}$ (a wavelength inside the substrate) and guarantees the needed phase delay to perform the desired beam steering with the frequency sweeping from 57 to $66 \mathrm{GHz}$.

Initially, all the ten slots are placed $-0.4 /+0.4 \mathrm{~mm}$ (odd/even slots, respectively) with respect to the array center along the $y$-direction (i.e., the slot feeding position, see Fig. 29), thus providing the same coupling level to all of them. After an iterative optimization process, by using the CST's trust region algorithm, defining a tradeoff between maximum achievable gain and a fixed value of SLLs below $-10 \mathrm{~dB}$, considering the whole frequency band from 57 to $66 \mathrm{GHz}$, the final position along $y$-direction for each individual slot is determined. A transversal pin curtains (see Fig. 29) are placed between slot elements in order to isolate each one from each other to avoid the coupling and suppressing the surface wave propagation between the parallel plates of the array. 


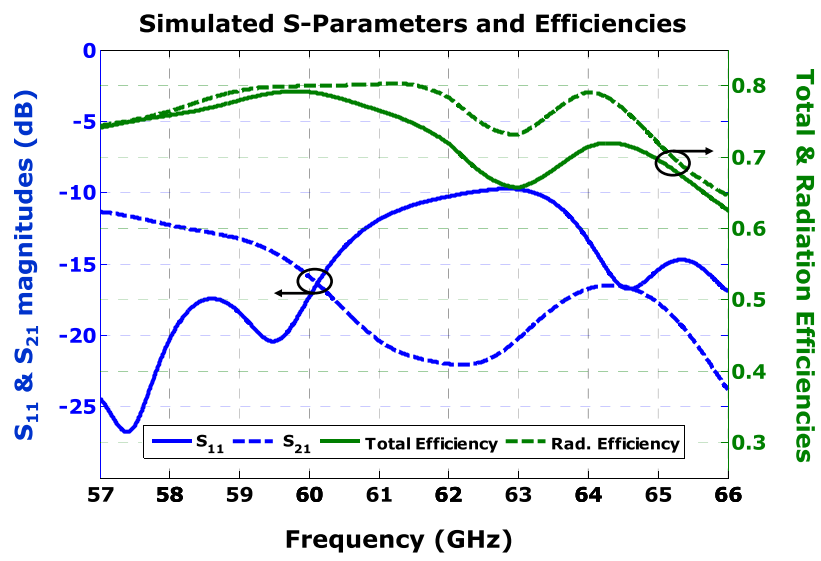

Fig. 30. S-Parameters $\left(S_{11}\right.$ and $\left.S_{21}\right)$ and efficiencies (total and radiation) simulation results for the frequency-scanned slot array in the wholeband of interest.

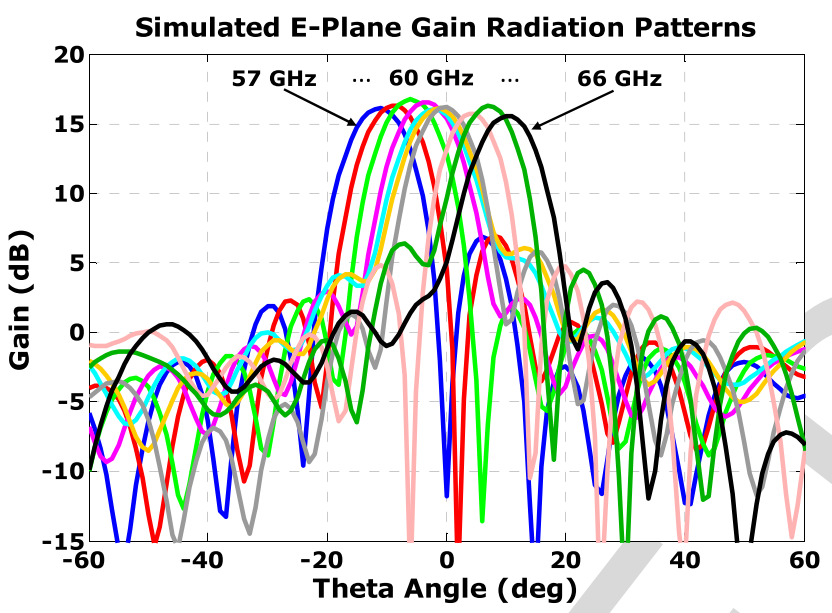

Fig. 31. Simulated E-plane gain radiation patterns obtained sweeping the frequency of the linear slot array, in steps of $1 \mathrm{GHz}$, from 57 to $66 \mathrm{GHz}$.

2) Frequency-Scanned Slot Array Simulation Results: Therefore, the final frequency-scanned slot array design has been simulated using CST Microwave Studio with the timedomain solver from 57 to $66 \mathrm{GHz}$. In Fig. 30, the simulation results of the S-parameters, radiation, and total efficiencies for the frequency-scanned array are plotted. As it is shown, the structure is well-matched since the reflection coefficient $\left(S_{11}\right)$ is below $-10 \mathrm{~dB}$ over the whole frequency band.

The simulated transmission coefficient $\left(S_{21}\right)$ is also below $-10 \mathrm{~dB}$, which means that most part of the input power is being transferred to the antenna from the feeding stripline, and then, radiated to the free-space; likewise, it is supposed that the power is not being trapped into the array structure. Moreover, in this sense, the simulated total and radiation efficiencies are showing values around $70 \%-80 \%$. Note that $S_{22}$ and $S_{12}$ parameters are not plotted due to the symmetry and reciprocity of the design.

The E-plane gain radiation pattern at each frequency, in steps of $1 \mathrm{GHz}$, is plotted in Fig. 31. As it is shown, with the proposed design we are able to scan the maximum of the beam from $-12^{\circ}$ to $+12^{\circ}$, with almost constant gain values

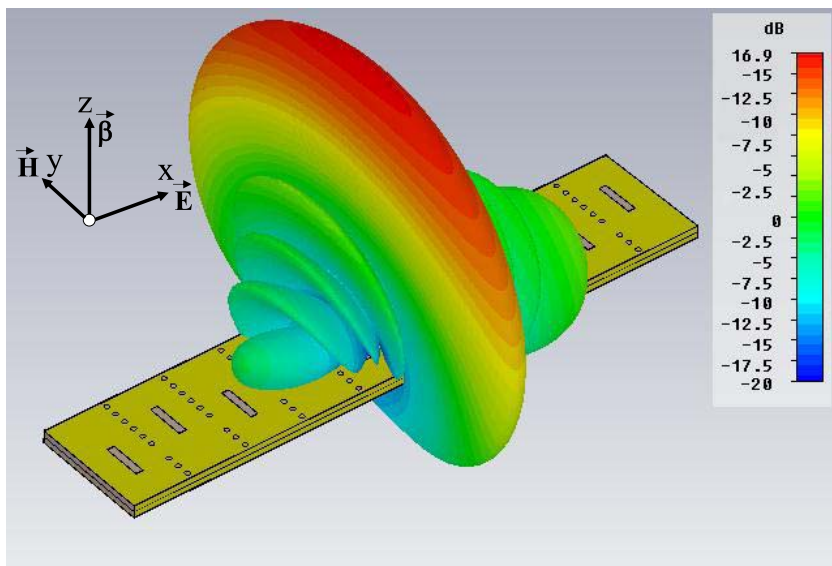

Fig. 32. 3-D representation of the fan-beam obtained in simulation with the frequency-scanned stripline-fed transverse slot antenna array at $60 \mathrm{GHz}$.

around $16 \mathrm{~dB}$, and up to $16.7 \mathrm{~dB}$ gain. From $-15^{\circ}$ to $+14^{\circ}$, we are able to obtain beam scanning with more than $15 \mathrm{~dB}$ gain, and from $-20^{\circ}$ to $+18^{\circ}$, we still have $10 \mathrm{~dB}$. SLL is below $-10 \mathrm{~dB}$ for all the radiation beams and below $-14 \mathrm{~dB}$ in most of the cases, with $-3 \mathrm{~dB}$ beamwidths around $12^{\circ}$.

Because of the linear distribution of the slots along $x$-direction, the frequency-scanned array is also generating a fan-beam radiation pattern having a narrow beamwidth in this specific dimension, while the typical broader beamwidth of a single slot antenna is obtained along the orthogonal $y$-axis, as it is expected. A 3-D representation of the fan-beam radiation pattern obtained with the numerical results of the performed simulations is plotted in Fig. 32. Note that since the linear array is modeled with a set of slot antennas individually linearly polarized in $x$-direction, the whole array structure is performing a linearly $x$-direction polarized radiation pattern as well.

The overall performance of the proposed slot array in simulation is comparable to the obtained with similar designs [22], having even better gain values while using a smaller fractional bandwidth to perform the frequency sweep.

Moreover, its singular novel stripline-fed transverse structure, with the feeding line isolated from outer parts, allows for a better control of the radiated fields in order to optimally illuminate the cylindrically distributed parameters flat lens, also facilitating an easier adaptation of the design if there is a change in the boundaries, or a redesign for higher frequencies is needed.

\section{Complete Switched-Beam Antenna Array}

This section is devoted to numerically evaluate the performance of the complete SWBA array structure based on both presented flat lens and frequency-scanned array.

1) Concept Description and Final Geometry: As it has been demonstrated before, with the help of the cylindrically distributed parameters flat lens it is possible to correct the phase in one single plane in order to focus the radiation beam. Since the FSSA provides a fan-beam radiation pattern, which is easy to steer along its linear structure by sweeping the 


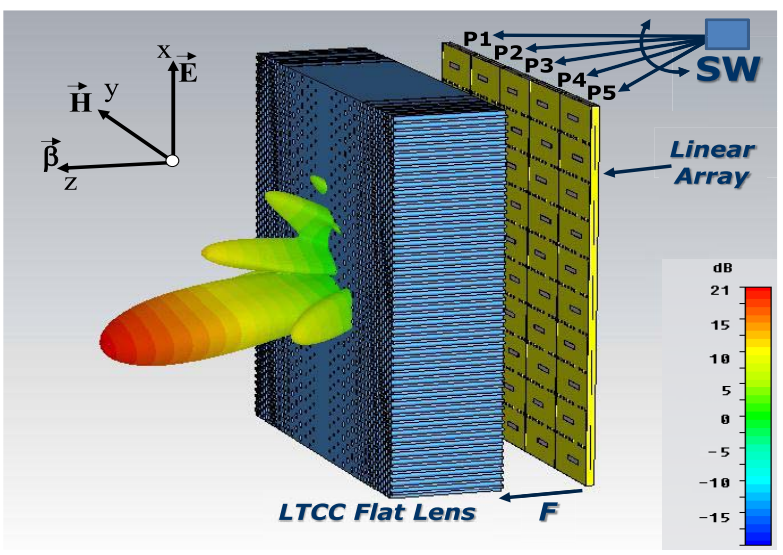

Fig. 33. Complete SWBA array structure for future high data rate 5G wireless communication applications and 3-D representation of the high-gain pencil beam obtained in numerical simulations.

frequency, if we correctly place this linear array orthogonally to the gradient-index axis of the lens, the final result will lead to a 2-D focused radiation beam, which in turn will allow the beam-scanning easily in 2-D. Therefore, the linear frequencyscanned array model has been replied five times along the gradient-index of the flat lens, placed orthogonally at its focal distance, as it is depicted in Fig. 33.

Since the overall dimensions of a single linear array are $5 \mathrm{~mm} \times 25 \mathrm{~mm}$, and because it is replied five times along $y$-axis (see Fig. 33), the final array planar dimensions are $25 \mathrm{~mm} \times 25 \mathrm{~mm}$, exactly the same square dimensions of the flat lens. The final structure is modeled with five input ports (P1-P5, Fig. 33), and five matched ports $(50 \Omega)$ at the end of each linear array. In this way, the number of switching elements needed if we want to individually select one single port among the five available is significantly reduced, thus in turn decreasing considerably the losses introduced and the complexity of the integration of this kind of electronic components at millimeter-wave frequencies.

2) Complete SWBA Simulation Results: The complete switched-beam antenna array structure has been numerically simulated with CST Microwave Studio in the $60 \mathrm{GHz}$ band, from 57 to $66 \mathrm{GHz}$, to evaluate the final performance of the proposed novel antenna solution. The corresponding E-plane gain radiation patterns obtained by sweeping the frequency from 57 to $66 \mathrm{GHz}$, in steps of $1 \mathrm{GHz}$, are plotted in Fig. 34, for the case of selecting the third port (i.e., the central linear array among the five).

As it is shown, with the proposed solution we are able to increase the maximum achievable gain up to $21.5 \mathrm{~dB}$, with constant gain level over $20 \mathrm{~dB}$, and beam scanning capabilities along the vertical dimension from $-12^{\circ}$ to $+12^{\circ}$ by sweeping the frequency from 57 to $66 \mathrm{GHz}$. SLL are below $-10 \mathrm{~dB}$ for all the beams, with narrow $-3 \mathrm{~dB}$ beamwidths around $11^{\circ}-12^{\circ}$.

The fan-beam radiation pattern generated by the FSSA array is modified by the gradient axis of the lens producing a highgain pencil-beam radiation pattern. A 3-D representation of the pencil-beam radiation pattern obtained with the numerical results of the performed simulations, together with the SWBA

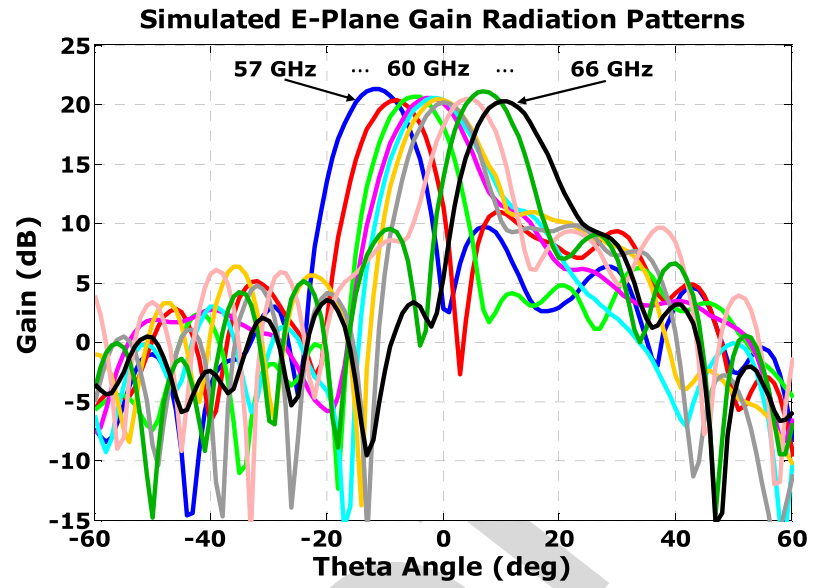

Fig. 34. Simulated E-plane gain radiation patterns obtained sweeping the frequency of P3 of the SWBA array, in steps of $1 \mathrm{GHz}$, from 57 to $66 \mathrm{GHz}$.

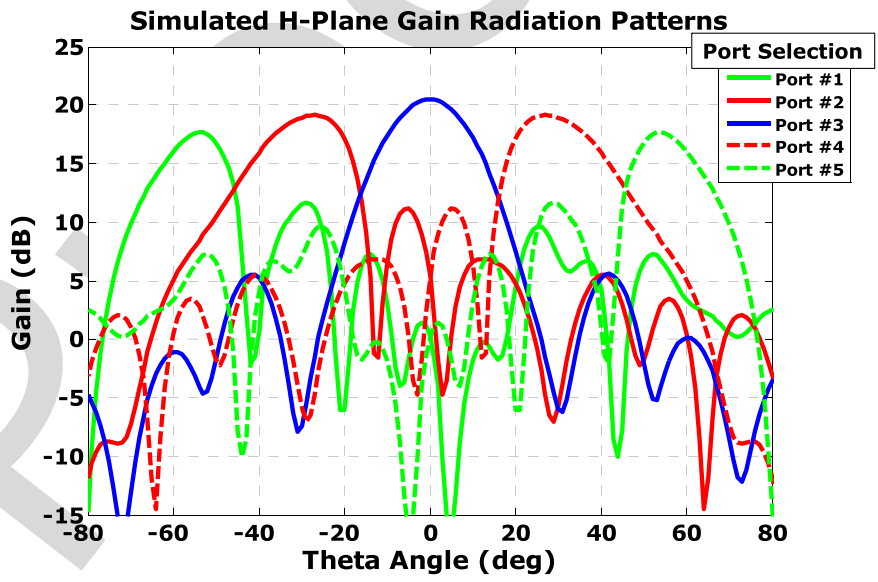

Fig. 35. Simulated H-plane gain radiation patterns at $61 \mathrm{GHz}$ obtained selecting individually each one of the five ports of the SWBA array.

array structure, is plotted in Fig. 33. Theoretically, an infinite number of high-gain pencil beams can be obtained to scan in the vertical direction, while in the horizontal dimension we can pick one of the five different sets of beams, depending on which one of the five ports of the array is selected, as it is plotted in Fig. 35, where the radiation patterns in the H-plane are shown at a frequency of $61 \mathrm{GHz}$ (in which the beams are pointing at $0^{\circ}$ in elevation), to finally cover the scanning in both azimuth and elevation.

In this sense, and in order to show the complete scanning capabilities of the SWBA array, a 3-D representation of the simulated gain radiation patterns obtained selecting individually ports \#3, \#2, and \#1, and changing the frequency at each port to 57,60 , and $66 \mathrm{GHz}$ (low, mid, and high band frequencies, respectively) are plotted in Fig. 36. Given the SWBA array symmetric structure, symmetric radiation patterns pointing rightwards in azimuth are obtained selecting ports \#4 and \#5 instead of ports \#1 and \#2, and therefore are not shown. Alternatively, the complete set of radiation patterns obtained selecting individually each one of the five ports, and sweeping the frequency from 57 to $66 \mathrm{GHz}$, in steps of $1 \mathrm{GHz}$ (ten patterns) at each port, is jointly plotted in Fig. 37. 

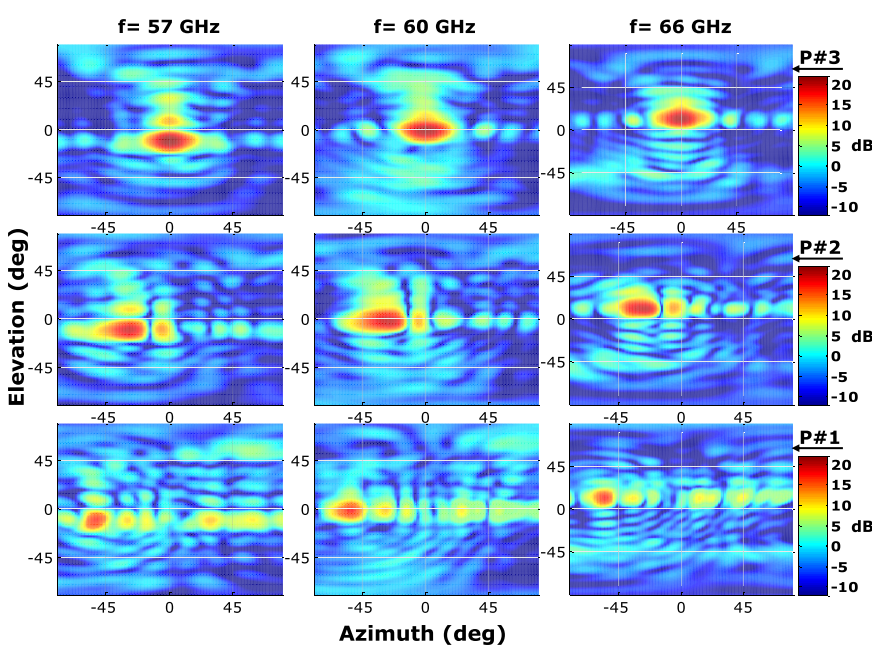

Fig. 36. 3-D representation of the simulated gain patterns obtained with the SWBA selecting individually ports \#3 (first row of the plot), \#2 (second row), and \#1 (third row) at single frequencies of 57, 60, and $66 \mathrm{GHz}$ (columns 1-3).

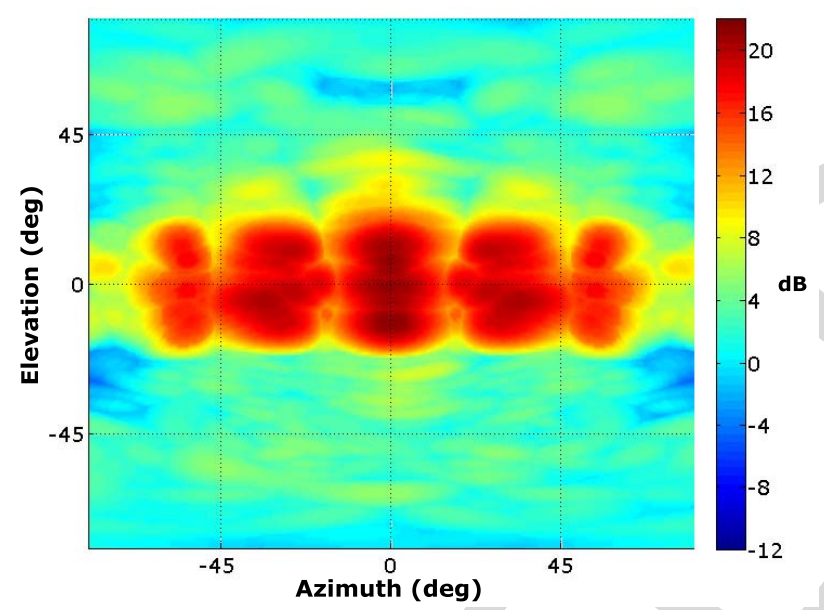

Fig. 37. 3-D joint representation of the complete set of simulated gain radiation patterns obtained with the SWBA selecting each one of the five ports (to scan over azimuth), and sweeping the frequency from 57 to $66 \mathrm{GHz}$ in steps of $1 \mathrm{GHz}$ at each port (to scan over elevation).

As it is observed in Figs. 36 and 37, our numerical results indicate that we are able to scan a high-gain radiation pencil beam (up to $21-21.5 \mathrm{~dB}$ in the broadside direction) from around $-55^{\circ}$ to $+55^{\circ}$ in azimuth, by selecting one single port of the five available, and from around $-20^{\circ}$ to $+20^{\circ}$ in elevation, by sweeping the frequency from 57 to $66 \mathrm{GHz}$ (the maximum of the beams in elevation is going from $-12^{\circ}$ to $+12^{\circ}$, as it is clearly shown in Fig. 34 , but at $\pm 20^{\circ}$ we still achieve up to $15 \mathrm{~dB}$ gain. The simulation results also indicate that the whole structure is well matched $\left(S_{11}<10 \mathrm{~dB}\right)$ for the entire frequency band, as it was expected, obtaining the same simulation results as the previously reported or the single FSSA array alone (thus are not plotted), because the lens, which is placed at $6.25 \mathrm{~mm}$ (focal distance) from the slot array, is not altering or modifying the FSSA array behavior in this sense. Likewise, simulated total and radiation efficiencies results are also quite similar to the previously reported for the

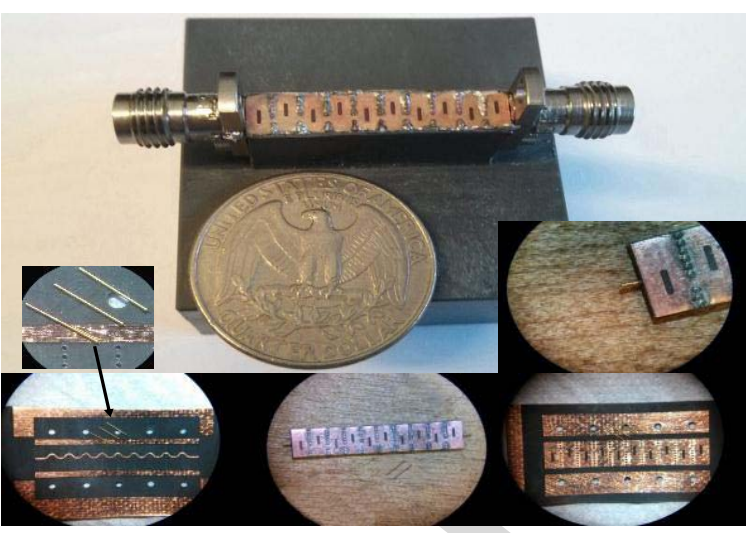

Fig. 38. FSSA connectorized and mounted over a PVC support. Some microscopic images of the bottom layer before being stacked (with the meandering stripline), complete design before connectorization, top layer (with the slots), and detailed image of the signal pin together with the first two slots and the first pin curtain, are shown in the insets.

FSSA array evaluated individually, since a low-loss substrate is used to model the lens, and therefore are not shown either.

\section{FSSA and SWBA Array Prototypes Fabrication}

1) Traveling-Wave Frequency-Scanned Slot Antenna Array: A prototype of the FSSA array has been fabricated at UPC facilities using standard photo-etching techniques on two Rogers Duroid 5880 substrate layers of $254 \mu \mathrm{m}$ thickness. All FSSA array dimensions are specified in previous sections of this paper. A photograph of the fabricated prototype, mounted over a PVC support to facilitate its electromagnetic characterization with our measurement setup, is shown in Fig. 38.

A low insertion loss $1.85 \mathrm{~mm}$ flange jack connector is mated to each signal pin of the FSSA array. The transversal pin curtains are made from $0.2 \mathrm{~mm}$ diameter brass rivets, which are separated $0.5 \mathrm{~mm}$ center to center; they are arranged in line, as it is depicted in Fig. 29, in two different groups of six and three pins, leaving a central space between them to allow the meandering stripline pass through. The pins are soldered interconnecting the top plane, in which the slots are printed, to the bottom ground plane, going through the two substrates.

2) Complete Switched Beam Antenna Array: Finally, the FSSA structure for the complete SBWA array, in which the single array is five times replied along its short dimension, has also been fabricated. A photograph of the prototype also mounted on a PVC support is shown in Fig. 39.

Therefore, the complete SWBA array structure, and the different parts of the final design (e.g., the five input ports connectorized, with their corresponding matched resistors (r1-r5) soldered at the end of each meandering line) are identified, together with the cylindrically distributed permittivity lens placed over the array at its focal distance $F$ with the help of a Rohacell foam structure is also shown in Fig. 39.

\section{E. Complete SWBA Measurement Results}

A complete set of measurements have been carried out at UPC facilities in order to assess the performance of the proposed antenna solution for millimeter-wave applications. 


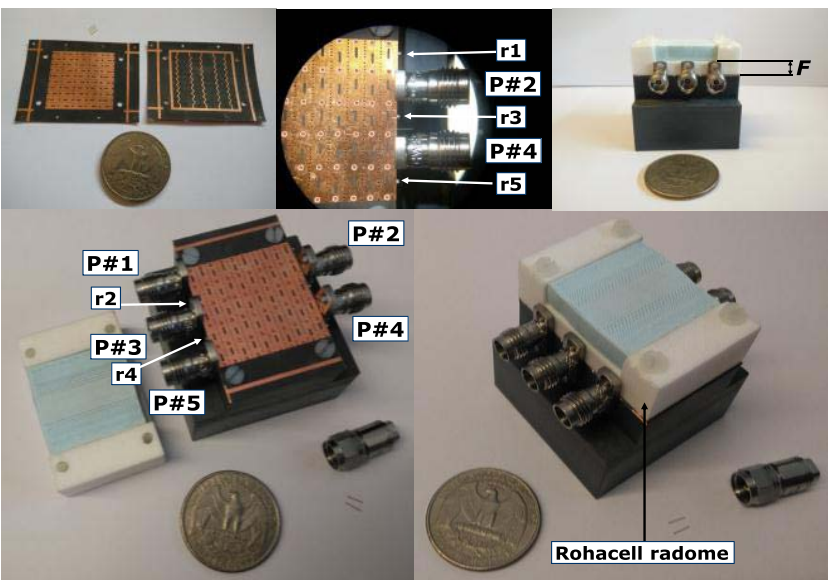

Fig. 39. Complete five-input port SWBA array for $60 \mathrm{GHz}$ WPAN applications, able to perform 2-D scanning of high-gain beams, mounted on a PVC support. Images of the five-input port FSSA fabrication process are shown in the insets.

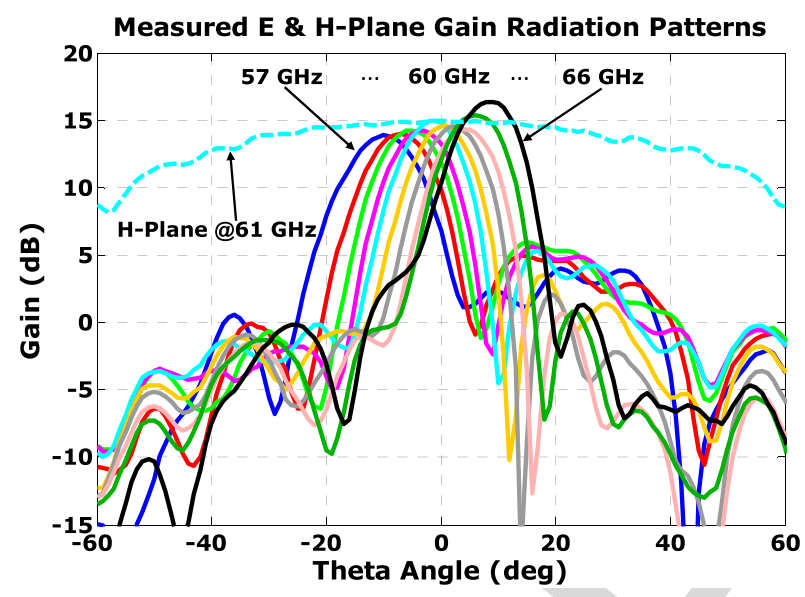

Fig. 40. Measured E-plane (solid lines) and H-plane (dashed lines) radiation patterns of the FSSA sweeping the frequency, from 57 to $66 \mathrm{GHz}$, in steps of $1 \mathrm{GHz}$.
First, the FSSA array is characterized separately and, finally, the complete SWBA array structure is completely tested in the whole $60 \mathrm{GHz}$ WPAN frequency band.

1) FSSA array Performance Evaluation: In the same way as it has been previously realized, the radiation pattern measurements for the FSSA array have been carried out at AntennaLab facilities of the UPC with the same far-field setup depicted in Fig. 11. The measured E-plane gain radiation patterns obtained from 57 to $66 \mathrm{GHz}$, in steps of $1 \mathrm{GHz}$, are plotted in Fig. 40. As it is observed, with the fabricated prototype we are able to scan the maximum of the beam from $-10^{\circ}$ to $+9^{\circ}$, with remarkable gain values above $14 \mathrm{~dB}$ for all scanning angles, with a maximum of $16.4 \mathrm{~dB}$ at $66 \mathrm{GHz}$, with $10^{\circ}$ of beam steering. Moreover, we are able to scan the radiation beam from $-18^{\circ}$ to $+16^{\circ}$ with at least $10 \mathrm{~dB}$ gain. SLL are below $-10 \mathrm{~dB}$ in most of the cases and around $-8.5 \mathrm{~dB}$ in the worst case, at $59 \mathrm{GHz}$, with $-3 \mathrm{~dB}$ beamwidths between $11^{\circ}$ and $13^{\circ}$.

The measured H-plane gain radiation pattern, which is the typical broad radiation pattern obtained for a single slot antenna, as it was expected, is also plotted (dashed line)

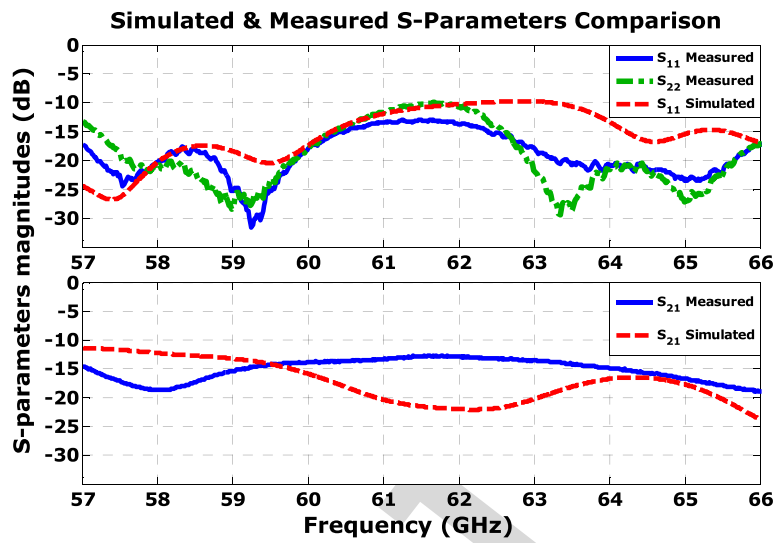

Fig. 41. Simulated and measured S-parameters comparison for the FSSA array in the whole frequency band of interest.

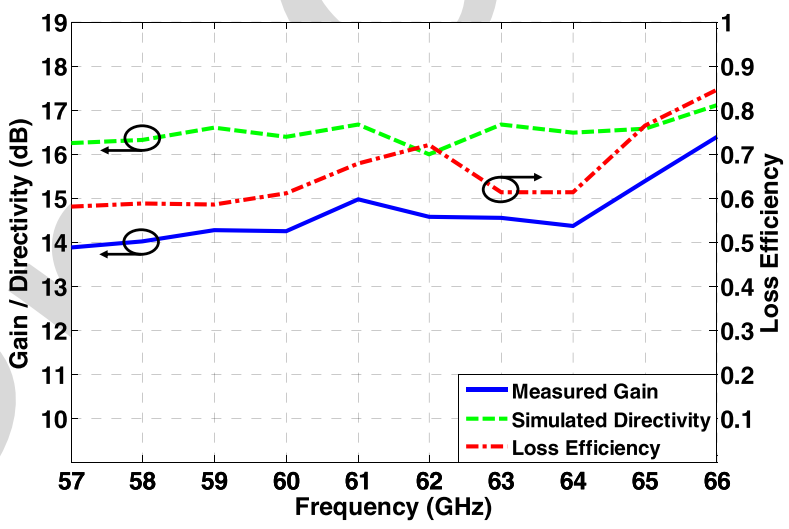

Fig. 42. Computed loss efficiency by using measured gain and simulated directivity results of the FSSA array in the whole frequency band of interest.

in Fig. 40, for a frequency of $61 \mathrm{GHz}$, in which the beam is pointing at $0^{\circ}$ in elevation, thus allowing the measurement in the $x z$ plane with our setup (see Fig. 11). Additionally, the measured cross-polarization level of the FSSA array is around $-20 \mathrm{~dB}$ below copolarization level.

The measured S-parameters of the FSSA array, after applying a full two-port SOLT calibration in the Agilent N5247A VNA, are plotted in Fig. 41 for the whole frequency band of interest. As it is shown, there is a very good agreement between simulation and measurement results; the FSSA is well matched and, since the measured transmission coefficient $\left(S_{21} / S_{12}\right)$ is below $-10 \mathrm{~dB}$, it is supposed that most part of the power is being radiated from the slots to the freespace, as we previously pointed out.

Going further in this sense, the estimated loss efficiency is plotted in Fig. 42, also computed from CST simulation results of the directivity, and measured gain values in the whole WPAN frequency band. The efficiency values, above $60 \%$, and up to $80 \%$, confirm the hypothesis that most part of the power is being correctly radiated. Also note that the measured $S_{11}$ and $S_{22}$ parameters are not identical because of small imperfections in the FSSA array fabrication process.

2) Complete Switched-Beam Antenna Array Characterization: In this section, the electromagnetic characterization of 


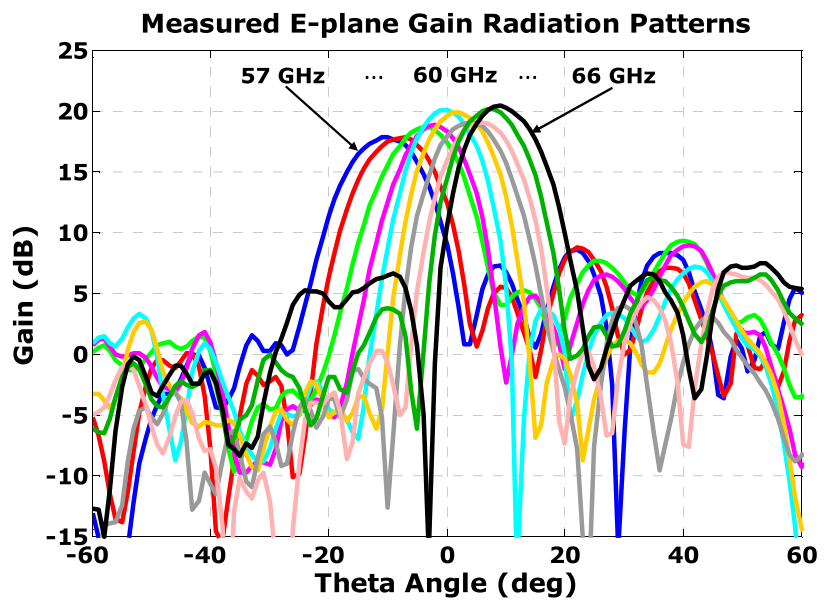

Fig. 43. Measured E-plane gain radiation patterns obtained sweeping the frequency, from 57 to $66 \mathrm{GHz}$, in steps of $1 \mathrm{GHz}$, selecting the third port (central linear array) of the SWBA array.

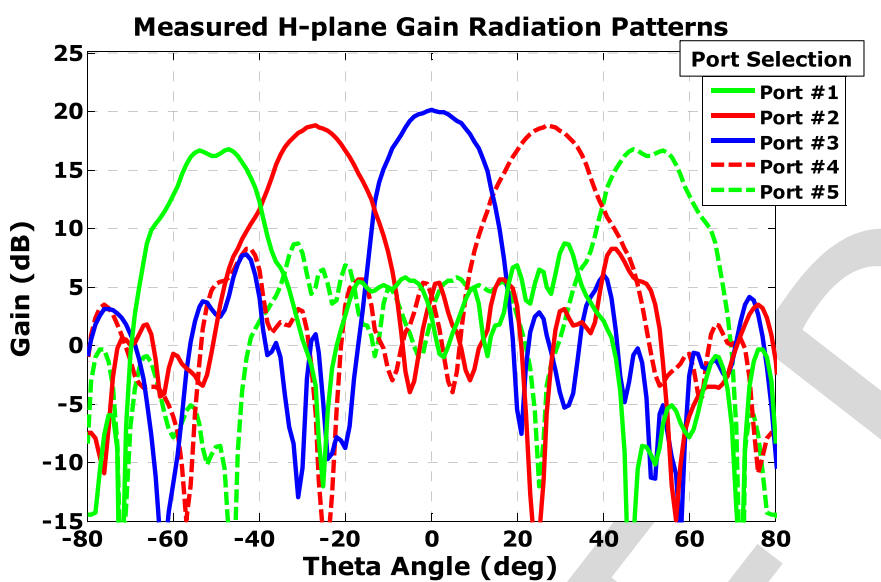

Fig. 44. Measured H-plane gain radiation patterns obtained at a frequency of $61 \mathrm{GHz}$, selecting individually each one of the five ports of the SWBA array.

the complete SWBA array structure, based on the previously presented and evaluated dielectric flat lens and FSSA array in its complete five-input port configuration, has also been carried out at AntennaLab facilities. A photograph of the final SWBA array prototype mounted on a PVC-Rohacell support to facilitate the measurements is shown in Fig. 39.

Similarly, as in previous sections, the gain radiation patterns of the SWBA array have been measured for different antenna configurations, from 57 to $66 \mathrm{GHz}$, in steps of $1 \mathrm{GHz}$, with the setup shown in Fig. 11. The E-plane gain radiation patterns obtained selecting the third port (central linear array of the five available), and sweeping the frequency are plotted in Fig. 43. The H-plane radiation patterns obtained selecting each one of the five ports separately, at a fixed frequency of $61 \mathrm{GHz}$ in which the beams are pointing $0^{\circ}$ in elevation, thus having their maximums in the $x z$ plane, are plotted in Fig. 44.

As it is observed in Fig. 43, by sweeping the frequency, with the fabricated SWBA array prototype we are able to scan the maximum of the beam from $-10^{\circ}$ to $+9^{\circ}$, with highgain values around $18 \mathrm{~dB}$ and above for all the scanning
TABLE VIII

Summary of SWBA ARray Performance at $60 \mathrm{GHz}$ Band Selecting Port \#3 (CEntral Port)

\begin{tabular}{|c|c|c|c|c|}
\hline Frequency & $\mathbf{G}_{\text {max. }}$ & $\left(\boldsymbol{\theta}^{\circ}\right)_{\text {scan }}$ & $\Delta \theta_{-3 \mathrm{~dB}}$ & SLL \\
\hline $57 \mathrm{GHz}$ & $17.8 \mathrm{~dB}$ & $-10^{\circ}$ & $13^{\circ}$ & $-9.3 \mathrm{~dB}$ \\
\hline $58 \mathrm{GHz}$ & $17.79 \mathrm{~dB}$ & $-7^{\circ}$ & $12.5^{\circ}$ & $-9.25 \mathrm{~dB}$ \\
\hline $59 \mathrm{GHz}$ & $18.7 \mathrm{~dB}$ & $-4^{\circ}$ & $12^{\circ}$ & $-9.4 \mathrm{~dB}$ \\
\hline $60 \mathrm{GHz}$ & $18.81 \mathrm{~dB}$ & $-2^{\circ}$ & $11.5^{\circ}$ & $-9.9 \mathrm{~dB}$ \\
\hline $61 \mathrm{GHz}$ & $20.05 \mathrm{~dB}$ & $0^{\circ}$ & $11.5^{\circ}$ & $-12.95 \mathrm{~dB}$ \\
\hline $62 \mathrm{GHz}$ & $19.87 \mathrm{~dB}$ & $+2^{\circ}$ & $11.5^{\circ}$ & $-14 \mathrm{~dB}$ \\
\hline $63 \mathrm{GHz}$ & $19.03 \mathrm{~dB}$ & $+4^{\circ}$ & $11.5^{\circ}$ & $-15.1 \mathrm{~dB}$ \\
\hline $64 \mathrm{GHz}$ & $19.02 \mathrm{~dB}$ & $+6^{\circ}$ & $11.5^{\circ}$ & $-12.3 \mathrm{~dB}$ \\
\hline $65 \mathrm{GHz}$ & $20.14 \mathrm{~dB}$ & $+7^{\circ}$ & $11.5^{\circ}$ & $-13.9 \mathrm{~dB}$ \\
\hline $66 \mathrm{GHz}$ & $20.4 \mathrm{~dB}$ & $+9^{\circ}$ & $11.5^{\circ}$ & $-13 \mathrm{~dB}$ \\
\hline
\end{tabular}

angles, and up to $20.4 \mathrm{~dB}$ at $66 \mathrm{GHz}$, when the beam is 1111 steered at $+9^{\circ}$. It is also remarkable that for wider scanning angles, from $-21^{\circ}$ to $+20^{\circ}$, we still have at least $10 \mathrm{~dB}$ gain. SLL are, at least, below $-9.25 \mathrm{~dB}$ in the worst case, and below $-12 \mathrm{~dB}$ in general, with $-3 \mathrm{~dB}$ beamwidths between $11.5^{\circ}$ and $13^{\circ}$. To facilitate the reading, the measured radiation pattern parameters of the SWBA array (maximum gain for each beam $\left(G_{\max }\right)$, scanning angles $\left(\theta^{\circ}\right.$ scan $)$, halfpower beamwidths $\left(\Delta \theta_{-3} \mathrm{~dB}\right)$, and SLL) are summarized in Table VIII.

Additionally, the measured cross-polarization level is around $-20 \mathrm{~dB}$ below copolarization level, as in the case of the FSSA array, because the lens is not affecting in this sense the performance of the combination.

As it is also observed from Fig. 44, selecting each one of the five ports, we are able to scan a high-gain radiation beam from $-54^{\circ}$ to $+54^{\circ}$ in the azimuth plane, obtaining more than $16.5 \mathrm{~dB}$ for this wide scanning angle, and still having $10 \mathrm{~dB}$ gain at $\pm 65^{\circ}$. In the broadside direction we achieve a considerable value over $20 \mathrm{~dB}$ gain. Therefore, five different sets of high-gain radiation beams can be selected to scan in the azimuth plane from $-54^{\circ}$ to $+54^{\circ}$, while at the same time an infinite number of beams can be generated in the elevation plane to scan from $-10^{\circ}$ to $+9^{\circ}$ with around $18-20 \mathrm{~dB}$ gain. In general, very good agreement is observed between the obtained radiation pattern measurement results in both planes and the estimated in advance from numerical simulations. Thus, despite we are not able to measure the complete 3-D gain radiation patterns for the SWBA array, the 3-D representation of the complete set of simulated gain radiation patterns plotted in previous Figs. 36 and 37, seems to be an accurate estimation, since the observed agreement between measurements and simulations in the E-plane and $\mathrm{H}$-plane cuts is very good.

The reflection coefficients $\left(S_{11}, S_{22}, S_{33}, S_{44}\right.$, and $\left.S_{55}\right)$ of the SWBA array for the five input ports have also been measured, obtaining approximately the same measurement results as for the $S_{11}$ of the FSSA array plotted in Fig. 41, because the lens placed at focal distance is not affecting the performance in this sense, and therefore are not shown due to space constraints.

Finally, the estimated loss efficiency of the SWBA array is also reported in Fig. 45, computed again from CST simulation results of the directivity and measured gain values, since 


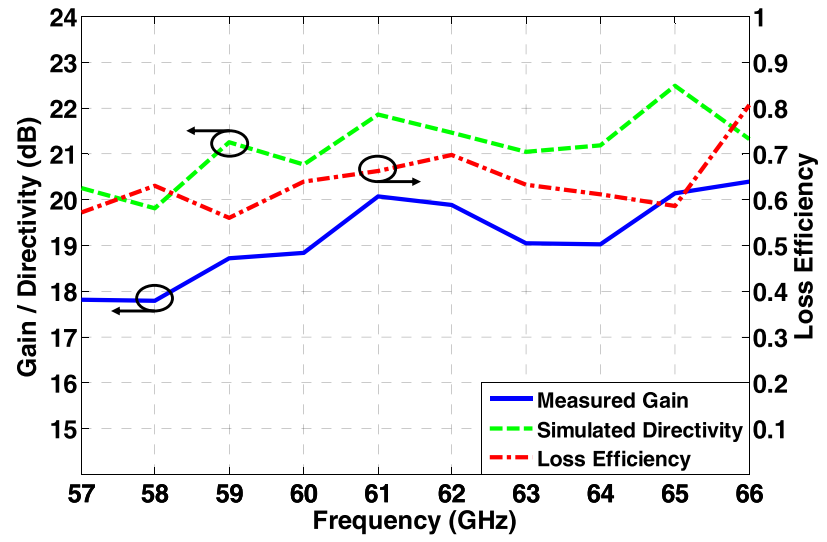

Fig. 45. Computed loss efficiency by using measured gain and simulated directivity results of the SWBA array in the whole frequency band of interest.

with our measurement setup we are not able to measure the complete 3-D radiation patterns in order to integrate the whole power to obtain directly the directivity or the efficiency. As it is observed, good values around $60 \%-70 \%$ and above are estimated in the whole frequency band of interest, also confirming the previously obtained simulation results. Moreover, very good bandwidth performance is also observed in Fig. 45, with gain slightly increasing in frequency, thus also validating the previously reported numerical results.

\section{CONCLUSION}

The design, numerical analysis, LTCC fabrication, and full experimental verification of new inhomogeneous gradientindex dielectric flat lens antennas for future high data rate 5G millimeter-wave wireless communication systems have been presented. Two novel dielectric flat lenses with their effective parameters circularly and cylindrically distributed to provide high-gain pencil-beam and fan-beam radiation patterns, respectively, are designed and fabricated in LTCC technology to allow beam-scanning along both theta and phi directions, despite their planar antenna profile implementation.

The two new LTCC dielectric flat lens antennas have been exhaustively evaluated and compared to a previously introduced TMM6 material flat lens [11], showing in all cases very good performance in terms of radiation pattern parameters: maximum measured gain (between 15 and $18 \mathrm{~dB}$ ), beamsteering capabilities in both planes (between approximately $-50^{\circ}$ and $+50^{\circ}$ ), and low SLL (below $-10 \mathrm{~dB}$ in most of the cases and below -15 and $-17.5 \mathrm{~dB}$ for the broadside direction); estimated efficiencies (over 70\%-80\%), impedance matching, and broadband behavior in the whole frequency band of interest $(57-66 \mathrm{GHz})$.

Additionally, a TDS system has been used to practically evaluate the permittivity profile achieved with the LTCC manufacturing process, which, to our best knowledge, has never been proved before, and even less stacking up to 31 layers of dielectric material, obtaining very good results to confirm the feasibility of fabricating inhomogeneous gradient-index lenses with a desired permittivity profile and planar structure in a mass production technology. The potential integration of the presented dielectric flat lenses in a complete antenna solution with a layer of radiating elements to create a single monolithic structure in LTCC technology has been confirmed as feasible.

Then, the performance of the considered lenses has also been experimentally evaluated and compared to a ten-element ULA of omni-directional antennas applying a beamforming technique, and to a single omni-directional antenna in real $60 \mathrm{GHz}$ WPAN indoor environment under LOS and OLOS conditions, obtaining remarkable results in terms of measured received power and RMS delay spread.

It has been practically demonstrated that in a real millimeter-wave communication scenario the best results in terms of relative received power are achieved in all the considered cases, despite the wide steering angle in which Rx antenna is placed respect to the Tx, with the TMM6 flat lens, closely followed by the circular LTCC lens, and in any case improving the results obtained with the ten-element ULA.

Moreover, the experimental analysis also indicate that in terms of RMS delay spread, the best results are obtained with the cylindrically distributed parameters flat lens, which provides a steerable fan-beam radiation pattern, a remarkable result because enhances the coherence bandwidth to improve the capacity in a wireless transmission system. In this sense, the measured RMS delay spread can be up to 15 times smaller using the proposed cylindrical LTCC flat lens compared to the RMS delay spread obtained with the virtual ULA, when, in a LOS situation, a wide angle between Tx and Rx is established.

Additionally, the complexity in the implementation of the proposed LTCC-based lens antenna solution, which is considerably lower compared to the difficulty in the implementation of beam-forming techniques for phased-array antennas, has also to be taken into account as an important point. It has been experimentally demonstrated their practical application as smart antenna solution for high data rate $5 \mathrm{G}$ millimeterwave commercial systems, not only for mobile devices such as tablets, laptops, or other similar medium-sized devices but also as a possible solution for APs, or even for outdoor BSs, due to their planar antenna configuration and 2-D scanning capability of high-gain radiation beams.

Finally, in order to propose and evaluate a practical application of the introduced lenses for an antenna system, a new switched beam antenna array concept based on the novel LTCC dielectric flat lens with the permittivity cylindrically distributed, and on a traveling-wave FSSA has been introduced, numerically investigated, fabricated, and successfully practically assessed for future $5 \mathrm{G}$ applications at $60 \mathrm{GHz}$ band. The dielectric flat lens and the frequency-scanned array have been exhaustively tested, first separately, and after that together as the complete SWBA array, showing in all cases very good performance in terms of radiation pattern parameters, beamsteering capabilities in both theta and phi planes, measured gain values, efficiencies, impedance matching, and broadband behavior in the whole frequency band of interest $(57-66 \mathrm{GHz})$.

The potential integration of the proposed complete antenna solution in a single monolithic structure has been demonstrated. This technology is suitable and allows mass production for a flat antenna structure such as the proposed in this paper, which is very interesting in order to integrate the solution in compact millimeter-wave wireless mobile devices. 
In contrast to other antenna alternatives, with the proposed solution we are able to scan high-gain radiation beams in both azimuth and elevation planes, necessary for supporting high data rate transmissions ( $>1.5 \mathrm{Gbps})$ as it is recommended in the IEEE 802.15.3c standard, and additionally avoiding the need of high number of integrated RF switches to perform such 2-D radiation pattern reconfiguration.

\section{REFERENCES}

[1] T. S. Rappaport et al., "Millimeter wave mobile communications for 5G cellular: It will work!" IEEE Access, vol. 1, pp. 335-349, May 2013.

[2] J. G. Andrews et al., "What will 5G be?" IEEE J. Sel. Areas Commun., vol. 32, no. 6, pp. 1065-1082, Jun. 2014.

[3] K. Chandra, R. V. Prasad, B. Quang, and I. G. M. M. Niemegeers, "CogCell: Cognitive interplay between $60 \mathrm{GHz}$ picocells and 2.4/5 GHz hotspots in the 5G era," IEEE Commun. Mag., vol. 53, no. 7, pp. 50-56, Jul. 2015.

[4] T. S. Rappaport, G. R. Maccartney, M. K. Samimi, and S. Sun, "Wideband millimeter-wave propagation measurements and channel models for future wireless communication system design," IEEE Trans. Commun., vol. 63, no. 9, pp. 3029-3056, Sep. 2015.

[5] R. Fisher, "60 GHz WPAN standardization within IEEE 802.15.3c," in Proc. Int. Symp. Signals, Syst. Electron. (ISSSE), Jul. 2007, pp. 103-105.

[6] S. Sun, G. R. MacCartney, M. K. Samimi, and T. S. Rappaport, "Synthesizing omnidirectional antenna patterns, received power and path loss from directional antennas for 5G millimeter-wave communications," in Proc. IEEE Global Commun. Conf. (GLOBECOM), San Diego, CA, USA, Dec. 2015, pp. 1-7.

[7] T. S. Rappaport, J. N. Murdock, and F. Gutierrez, Jr., "State of the art in 60-GHz integrated circuits and systems for wireless communications," Proc. IEEE, vol. 99, no. 8, pp. 1390-1436, Aug. 2011.

[8] J. R. Costa, E. B. Lima, and C. A. Fernandes, "Compact beamsteerable lens antenna for $60-\mathrm{GHz}$ wireless communications," IEEE Trans. Antennas Propag., vol. 57, no. 10, pp. 2926-2933, Oct. 2009.

[9] M. Imbert, A. Papio, F. De Flaviis, L. Jofre, and J. Romeu, "Design and performance evaluation of a switched-beam antenna array for $60 \mathrm{GHz}$ WPAN applications," in Proc. 9th Eur. Conf. Antennas Propag. (EuCAP), Apr. 2015, pp. 1-5.

[10] A. Artemenko, A. Maltsev, A. Mozharovskiy, A. Sevastyanov, V. Ssorin, and R. Maslennikov, "Millimeter-wave electronically steerable integrated lens antennas for WLAN/WPAN applications," IEEE Trans. Antennas Propag., vol. 61, no. 4, pp. 1665-1671, Apr. 2013.

[11] M. Imbert, A. Papió, F. D. Flaviis, L. Jofre, and J. Romeu, "Design and performance evaluation of a dielectric flat lens antenna for millimeterwave applications," IEEE Antennas Wireless Propag. Lett., vol. 14, pp. 342-345, 2015.

[12] A. Petosa and A. Ittipiboon, "Design and performance of a perforated dielectric Fresnel lens," IEE Proc.-Microw., Antennas Propag., vol. 150, no. 5, pp. 309-314, Oct. 2003.

[13] A.-E. Mahmoud, W. Hong, Y. Zhang, and A. Kishk, "W-band mutlilayer perforated dielectric substrate lens," IEEE Antennas Wireless Propag. Lett., vol. 13, pp. 734-737, 2014.

[14] M. K. T. Al-Nuaimi and W. Hong, "Discrete dielectric reflectarray and lens for E-band with different feed," IEEE Antennas Wireless Propag. Lett., vol. 13, pp. 947-950, 2014.

[15] P. F. M. Smulders, "Statistical characterization of $60-\mathrm{GHz}$ indoor radio channels," IEEE Trans. Antennas Propag., vol. 57, no. 10, pp. 2820-2829, Oct. 2009.

[16] A. Maltsev, R. Maslennikov, A. Sevastyanov, A. Khoryaev, and A. Lomayev, "Experimental investigations of $60 \mathrm{GHz}$ WLAN systems in office environment," IEEE J. Sel. Areas Commun., vol. 27, no. 8, pp. 1488-1499, Oct. 2009.

[17] J. George, P. F. M. Smulders, and M. H. A. J. Herben, "Application of fan-beam antennas for $60 \mathrm{GHz}$ indoor wireless communication," Electron. Lett., vol. 37, no. 2, pp. 73-74, Jan. 2001.

[18] W. Hong, K.-H. Baek, Y. Lee, Y. Kim, S.-T. Ko, "Study and prototyping of practically large-scale mmWave antenna systems for $5 \mathrm{G}$ cellular devices," IEEE Commun. Mag., vol. 52, no. 9, pp. 63-69, Sep. 2014.

[19] C. Q. Scrantom and J. C. Lawson, "LTCC technology: Where we are and where we're going. II," in IEEE MTT-S Int. Microw. Symp. Dig., Feb. 1999, pp. 193-200.
[20] M.-T. Martinez-Ingles, D. P. Gaillot, J. Pascual-García, J.-M. Molina-Garcia-Pardo, M. Liénard, and J.-V. Rodríguez, "Deterministic and experimental indoor $\mathrm{mmW}$ channel modeling," IEEE Antennas Wireless Propag. Lett., vol. 13, pp. 1047-1050, Dec. 2014.

[21] R. Hansen, Phased Array Antennas. New York, NY, USA: Wiley, 1998.

[22] L. Ranzani, N. Ehsan, and Z. Popović, "G-band frequency-scanned antenna arrays," in Proc. IEEE Antennas Propag. Soc. Int. Symp., Toronto, ON, Canada, Jul. 2010, pp. 1-4.

[23] W. F. Moulder, W. Khalil, and J. L. Volakis, "60-GHz two-dimensionally scanning array employing wideband planar switched beam network," IEEE Antennas Wireless Propag. Lett., vol. 9, pp. 818-821, 2010.

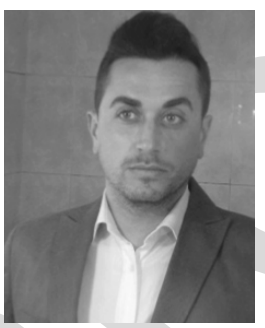

Marc Imbert ( $\mathrm{S}^{\prime}-$ ) was born in Calella (Barcelona), Spain. He received the Telecommunications Engineering degree, Master of Research on Information and Communications Technologies, and the $\mathrm{Ph} . \mathrm{D}$. degree from the Universitat Politècnica de Catalunya (UPC), Barcelona, in 2010, 2013, and 2016, respectively.

In 2009, he joined the Electromagnetic and Photonics Engineering Group (EEF), Department of Signal Theory and Communications, UPC, where he was involved in metamaterial research and its application to antennas. From 2010 to 2011, he was with the Urbiotica S.L, where he was responsible for electromagnetic analysis and antenna design. He was with the Department of Signal Theory and Communications, UPC, where he was involved in the frame of millimeter-wave antennas for communications, radar, and imaging, under the supervision of Professor Jordi Romeu. He was a Visiting Scholar with the University of California, Irvine, CA, USA, where he was with the High-Frequency Electronics Laboratory, under the supervision of Professor Franco De Flaviis. He is currently an RF/Antenna Engineer with the Ficosa Corporation, an automotive industry. His current research interests include metamaterial applications at microwave frequencies, lens antennas, embedded antennas, and electromagnetic characterization of small antennas.

Dr. Imbert was a recipient of the Spanish Government FPI fellowship, and finalist for the 2014 Best Paper Award in Antenna Applications at the European Conference on Antennas and Propagation.

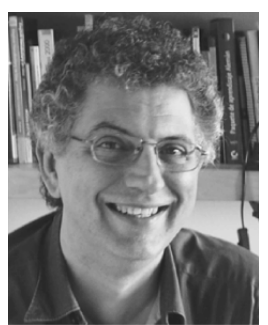

Jordi Romeu (F'12) was born in Barcelona, Spain, in 1962. He received the Ingeniero de Telecomunicación and Doctor Ingeniero de Telecomunicacíon degree from the Universitat Politècnica de Catalunya (UPC), Barcelona, Spain, in 1986 and 1991, respectively.

$\mathrm{He}$ has been with the Electromagnetic and Photonic Engineering Group, Signal Theory and Communications Department, UPC, since 1985, where he is currently a full Professor, where he is involved in the research of antenna near-field measurements, antenna diagnostics, and antenna design. He joined the Antenna Laboratory, University of California, Los Angeles, CA, USA, in 1999, as a Visiting Scholar, under the North Atlantic Treaty Organization Scientific Program Scholarship. In 2004, he joined the University of California, Irvine, CA, USA. He has authored 50 refereed papers in international journals and 50 conference proceedings and holds several patents.

Dr. Romeu received the Grand Winner of the European IT Prize by the European Commission, for his contributions in the development of fractal antennas in 1998. 


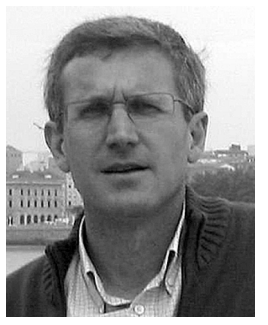

Mariano Baquero-Escudero (S'87-M'90) was born in Murcia, Spain, in 1962. He received the Telecommunications Engineering degree from the Polytechnic University of Catalonia (UPC), Barcelona, Spain, in 1986, and the Ph.D. degree from the Universitat Politècnica de València (UPV), València, Spain, in 1994

From 1986 to 1988, he was with the Antennas, Microwave and Radar Group, UPC, where he was involved in the development of a cylindrical nearfield facility to measure a 3-D radar antenna in CESELSA. Since 1989, he has been with the UPV, where he was a full Professor in 2003. During 1995, he held a Post-Doctoral grant with the Joint Research Center, European Commission, Ispra, Italy, where he developed high-resolution algorithms for radar applications. From 1996 to 1998, he was a Vice-Dean of the Telecommunications Engineering School of Valencia, Valencia. He is currently with the Communications Department, Institute of Telecommunications and Multimedia Application, UPV. His current research interests include microwave circuit and antenna analysis, design, and measurement.

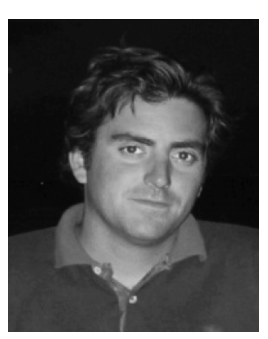

Jose-Maria Molina-Garcia-Pardo received the Engineer of Telecommunications degree from the Universidad Politécnica de Valencia, Valencia, Spain, in 2000, the M.Sc. degree in communication and signal processing in Newcastle upon Tyne, U.K. in 2001, and the Ph.D. degree in telecommunications from the Universidad Politécnica de Cartagena (UPCT), Cartagena, Spain, in 2004.

In 2001, he joined the Information Technologies and Communications Department, UPCT, where he was an Associate Professor in 2007 and has been a Full Professor since 2016. He is currently a Leader of the SICOMO Research Group. He is the Lead Researcher in some national projects, and participates actively in the European COST action IC-1004 (Radio Communications for Green Smart Environments). He has authored papers that have appeared in over 60 journals indexed in the JCR, over 100 international conferences, and three book chapters. His current research interests include centered on radio-communications, propagation, channel modeling, and experimental channel sounding in different frequency band $(400 \mathrm{MHz}-60 \mathrm{GHz})$, and technologies (GSM, UMTS, LTE, WiFi, WSN, TETRA, millimeterwave, OFDM, MIMO, and cognitive radio).

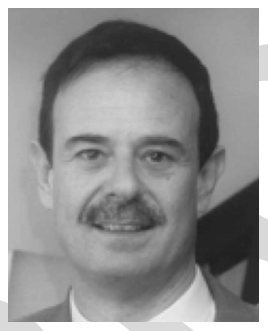

Lluis Jofre (S'79-M'83-SM'07-F'10) was born in Canet de Mar, Spain, in 1956. He received the M.Sc. (Ing.) and Ph.D. (Doctor Ing.) degrees in electrical engineering (telecommunication engineering) from the Technical University of Catalonia (UPC), Barcelona, Spain, in 1978 and 1982, respectively.

$\mathrm{He}$ was a Research Assistant with the Electrophysics Group, UPC, from 1979 to 1980, where he was involved in the analysis and near-field measurement of antennas and scatterers. From 1981 to 1982, he was with the École Supérieure d'Electricité Paris, Gif-sur-Yvette, France, where he was involved in microwave antenna design and imaging techniques for medical and industrial applications. Since 1982, he has been with the Communications Department, Telecommunication Engineering School, UPC, as an Associate Professor and then as a ful Professor since 1989. From 1986 to 1987, he was a Visiting Fulbright Scholar with the Georgia Institute of Technology, Atlanta, GA, USA, where he was involved in antennas and electromagnetic imaging and visualization. From 1989 to 1994, he was the Director of the Telecommunication Engineering School, UPC, and from 1994 to 2000, he was the UPC Vice-Rector for academic planning. From 2000 to 2001, he was a Visiting Professor with the Electrical and Computer Engineering Department, Henry Samueli School of Engineering, University of California, Irvine, CA, USA. From 2002 to 2004 he was the Director of the Catalan Research Foundation, and since 2003, he has been the Director of the UPC-Telefonica Chair and the Director of the Promoting Engineering Catalan Program EnginyCAT. He has authored more than 100 scientific and technical papers, reports, and chapters in specialized volumes. His current research interests include antennas, electromagnetic scattering and imaging, system miniaturization for wireless, and sensing industrial and bio-applications.

Dr. Jofre is a member of Different Higher Education Evaluation Agencies at Spanish and European level. Since 2010, he has been the General Director of Universities in the Economy and Knowledge Council of the Catalan Government. 


\section{AUTHOR QUERIES}

\section{AUTHOR PLEASE ANSWER ALL QUERIES}

PLEASE NOTE: We cannot accept new source files as corrections for your paper. If possible, please annotate the PDF proof we have sent you with your corrections and upload it via the Author Gateway. Alternatively, you may send us your corrections in list format. You may also upload revised graphics via the Author Gateway.

AQ:1 = Please provide the section number which represents the last section.

AQ:2 = Please provide the section numbers which represent the following sections.

AQ:3 = Please provide the section number which represents the previous section.

AQ:4 = Please provide the section number which represents the previous section.

AQ:5 = Please provide the section numbers which represent the previous sections.

AQ:6 = Please provide the section numbers which represent the following sections.

AQ:7 = Please provide the expansion for the acronyms "VNA, SISO, SWBA, and PVC."

$\mathrm{AQ}: 8=$ Please provide the section numbers which represent the following sections.

$\mathrm{AQ}: 9=$ Please provide the section numbers which represent the previous sections.

AQ:10 = Please provide the section numbers which represent the previous sections.

AQ:11 = Please provide the section number which represents the last section.

AQ:12 = Please provide the section number which represent the final paper section.

AQ:13 = Please provide the section numbers which represent the previous sections.

AQ:14 = Please provide the section numbers which represent the previous sections.

AQ:15 = Please note that there were discrepancies between the accepted pdf

[imbert.et.al.TAP_special_issue_5G_R2.pdf] and the [FINAL VERSION.docx] in the references. We have followed [FINAL VERSION.docx].

AQ:16 = Please provide the missing IEEE membership year for the author "Marc Imbert."

AQ:17 = Please provide the locations for "Urbiotica S.L, Ficosa Corporation, SICOMO Research Group, Catalan Research Foundation, and CESELSA." 


\title{
Assessment of LTCC-Based Dielectric Flat Lens Antennas and Switched-Beam Arrays for Future 5G Millimeter-Wave Communication Systems
}

\author{
Marc Imbert ${ }^{\circledR}$, Student Member, IEEE, Jordi Romeu, Fellow, IEEE, Mariano Baquero-Escudero, Member, IEEE, \\ Maria-Teresa Martinez-Ingles, Jose-Maria Molina-Garcia-Pardo, and Lluis Jofre, Fellow, IEEE
}

\begin{abstract}
This paper presents the design, low-temperature co-fired ceramics (LTCC) fabrication, and full experimental verification of novel dielectric flat lens antennas for future high data rate $5 \mathrm{G}$ wireless communication systems in the $60 \mathrm{GHz}$ band. We introduce and practically completely evaluate and compare the performance of three different inhomogeneous gradient-index dielectric lenses with the effective parameters circularly and cylindrically distributed. These lenses, despite their planar profile antenna configuration, allow full 2 -D beam scanning of high-gain radiation beams. A time-domain spectroscopy system is used to practically evaluate the permittivity profile achieved with the LTCC manufacturing process, obtaining very good results to confirm the viability of fabricating inhomogeneous flat lenses in a mass production technology. Then, the lenses performance is evaluated in terms of radiation pattern parameters, maximum gain, beam scanning, bandwidth performance, efficiencies, and impedance matching in the whole frequency band of interest. Finally, the performance of the three lenses is also experimentally evaluated and compared to a single omni-directional antenna and to a ten-element uniform linear array of omni-directional antennas in real $60 \mathrm{GHz}$ wireless personal area network indoor line-of-sight (LOS) and obstructed-LOS environments, obtaining interesting and promising remarkable results in terms of measured received power and root-mean-square delay spread. At the end of this paper, an innovative switched-beam antenna array concept based on the presented cylindrically distributed effective parameters lens is also introduced and completely evaluated, confirming the potential applicability of the proposed antenna solution for future 5G wireless millimeter-wave communication system.
\end{abstract}

Index Terms-5G, $60 \mathrm{GHz}$ band, beam steering, delay spread, flat lens antennas, inhomogeneous lenses, low-temperature

Manuscript received September 21, 2016; revised October 9, 2017; accepted October 19, 2017. This work was supported in part by the Spanish InterMinisterial Commission on Science and Technology (CICYT) under Project TEC2013-47360-C3-1-P and Project TEC2013-47360-C3-2-P and in part by the "Ministerio de Economía" through the FPI Fellowship Program. (Corresponding author: Marc Imbert.)

M. Imbert, J. Romeu, and L. Jofre are with the AntennaLab Research Group, Department of Signal Theory and Communications, Universitat Politècnica de Catalunya, 08034 Barcelona, Spain (e-mail: marc.imbert@tsc.upc.edu).

M. Baquero-Escudero is with the Instituto de Telecomunicaciones y Aplicaciones Multimedia, Universitat Politècnica de València, 46022 València, Spain (e-mail: mbaquero@dcom.upv.es).

M.-T. Martinez-Ingles is with the University Center of Defense, San Javier Air Force Base, MDE-UPCT, 30720 Murcia, Spain.

J.-M. Molina-Garcia-Pardo is with the Departamento de Tecnologías de la Información y las Comunicaciones, Universidad Politécnica de Cartagena, 30202 Murcia, Spain (e-mail: josemaria.molina@upct.es).

Color versions of one or more of the figures in this paper are available online at http://ieeexplore.iee.org.

Digital Object Identifier 10.1109/TAP.2017.2767821 co-fired ceramics (LTCC), millimeter-wave antennas, power delay profile (PDP), smart antennas, switched-beam arrays, wireless personal area network (WPAN).

\section{INTRODUCTION}

$\mathbf{T}$ HE future broadband wireless communication systems will have the need for more bandwidth in order to satisfy the increasing demands to achieve higher data rates. In this sense, the millimeter-wave frequency band will play a key role in fifth generation $(5 \mathrm{G})$ wireless cellular networks [1]-[3].

Four different frequency bands around 28, 38, 60, and $73 \mathrm{GHz}$ have been considered in the millimeter-wave region as perfect candidates for future $5 \mathrm{G}$ mobile communication systems in both indoor and outdoor environments [3], [4]. Actually, wireless personal area networks (WPANs) for highspeed data rate short-range communications around $60 \mathrm{GHz}$ band (from 57 to $64 \mathrm{GHz}$ in the United States, and up to $66 \mathrm{GHz}$ in Europe [5]), have attracted growing attention from the scientific community and industry in the last years. This huge amount of bandwidth available could allow the development of high throughput transmission systems for the future $5 \mathrm{G}$ cellular networks. However, at millimeter-wave frequencies, the path loss in free-space propagation is considerably higher than at lower microwave frequencies (for example, the attenuation is up to $28 \mathrm{~dB}$ higher at $60 \mathrm{GHz}$ compared to at $2.45 \mathrm{GHz}$, for a fixed transmission distance). Therefore, in order to allow future $5 \mathrm{G}$ millimeter-wave devices to achieve high data rate wireless transmissions, from the antenna point of view, it is absolutely necessary to dispose of high-directive antennas to overcome the aforementioned huge path loss attenuation. Additionally, antennas with certain beam-steering capabilities are also desirable in order to facilitate the reconfiguration of the radiation beam in situations of transmission blockage between devices in line-of-sight (LOS), obstructedLOS (OLOS), or even in non-LOS (NLOS). Moreover, beamsteerable adaptive antennas for $5 \mathrm{G}$ systems are not yet conveniently available at most millimeter-wave frequencies, even for researchers in order to measure and characterize the channel at a wide frequency range [6].

So far, many types of antenna structures have been proposed for millimeter-wave wireless communication systems around $60 \mathrm{GHz}$ frequency band [7], most of them based on the complex phased-array antenna concept. With this antenna solution, 
high-gain radiation beams can be scanned in two-dimensions at a fast rate. However, they require a difficult integration of some complex, lossy, and bulky components such solid-state phase shifters, making this antenna alternative very expensive at high frequencies for consumer mobile devices.

Aperture antennas, such as profiled lenses, rectangular or conical horns, and reflectors are traditional antenna solutions at millimeter-wave frequencies for communications, radar, and imaging applications due to their high gain and wide bandwidth. However, most common apertures with beamscanning capabilities result in a large and volumetric antenna configuration not suitable for consumer mobile devices (e.g., a homogeneous profiled lens illuminated by a conical horn antenna with a mechanical system to steer the radiation beam in two-dimensions [8]), or their planar implementation allow only 1-D beam steering, instead of 2-D.

Consequently, in [9], we introduced a planar profile antenna configuration based on the switched-beam array antenna concept (see [10]) with an inhomogeneous gradient-index dielectric flat lens to steer and enhance the radiation in a specific direction, achieving a 2-D beam scanning of high-gain radiation beams while maintaining a completely flat antenna profile very suitable for medium-sized mobile devices. The novel inhomogeneous flat lens design used in the switchedbeam antenna array was introduced, fabricated, and electromagnetically characterized in [11].

Therefore, compared to previously published works, in this paper, we introduce design, numerical simulation, novel fabrication in low-temperature co-fired ceramics (LTCC) technology, full experimental verification, and practical application of two new inhomogeneous gradient-index dielectric flat lenses for future high data rate $5 \mathrm{G}$ wireless communication systems in the $60 \mathrm{GHz}$ band. The performance of these lenses, which have their effective dielectric parameters circularly and cylindrically distributed, is also compared to the aforementioned lens presented in [11], in terms of radiation pattern parameters, highest achievable gain, beam-scanning capabilities in both theta and phi dimensions, bandwidth performance, efficiencies, and impedance matching over the whole frequency band of interest. Then, the performance of the three lenses is also experimentally evaluated and compared to a single omni-directional antenna and to a ten-element uniform linear array (ULA) of omni-directional antennas in a real $60 \mathrm{GHz}$ WPAN indoor environment under LOS and OLOS conditions, in terms of measured received power and rootmean-square (RMS) delay spread [15], [16], to evaluate their practical application as smart antenna solutions for high data rate $5 \mathrm{G}$ millimeter-wave systems, not only for mobile devices but also as a possible solution for access points (APs) [17], or even for outdoor base stations (BS), due to their flat antenna configuration and 2-D scanning capability of highgain radiation beams. Finally, in the last section of this paper, we also introduce a new switched-beam antenna array concept based on a novel cylindrically distributed parameters flat lens, which has an effective gradient-index in one axis, while a constant index is maintained along the other one. With this cylindrical effective parameter distribution, the beam scanning can be performed in one plane by moving (or selecting) the

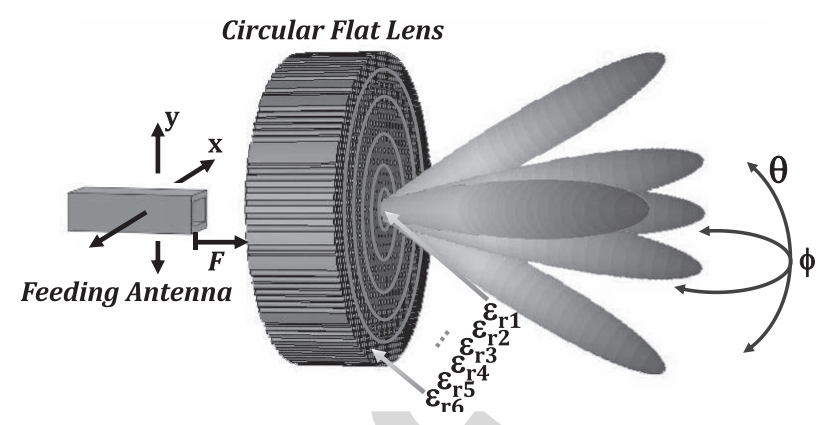

Fig. 1. Circularly distributed parameters flat lens concept and modeling by using triangular unit cells of perforations.

position of a radiating single element along the gradient-index axis, whereas the beam can be maintained invariant in the other direction, in which the effective parameters are kept constant, despite changing the radiating element position in this particular axis. In this way, the beam scanning can be achieved in the constant-index axis of the lens by means of a different technique, a frequency-scanned slot antenna array (FSSA), which it is also introduced at the end of this paper, in order to reduce the switching elements needed in the proposed complete switched-beam antenna array structure, to finally perform the scan of the high-gain radiation beam in both theta and phi dimensions of the space.

\section{Flat Lenses Design and Simulation Results}

Two different new inhomogeneous gradient-index dielectric flat lenses are designed and numerically simulated, each one with its particular effective parameters distribution, in order to obtain two different radiation pattern characteristics and beam-steering capabilities. In this sense, we are interested in achieving two different high-gain beam shapes: a pencil-beam and a fan-beam radiation patterns, because depending on the situation they have been experimentally proved as attractive solutions in the millimeter-wave frequency band for indoor communications [17] and 5G systems [18].

\section{A. Concept Description}

The particular parameters in both lens designs are optimized previously considering the constraints and difficulties in the fabrication of inhomogeneous lenses. Regarding this point, we investigated the possibility of fabricating the designs in a mass production technology such as LTTC technology. Therefore, in the following sections, the concept description and design considerations are defined taking into account the viability in the subsequently prototype fabrication.

1) Circularly Distributed Parameters Flat Lens Concept: The inhomogeneous gradient-index circular flat lens operating principle and design procedure are completely described in [11], and the theoretical concept is depicted in Fig. 1.

Fundamentally, the design consists of a set of six concentric rings of different permittivity $\left(\varepsilon_{r}\right)$ materials, in order to produce the desired phase delays required to obtain a plane wave behind the lens, when the lens is illuminated from its central focus position. In the same way, when the feeding position 


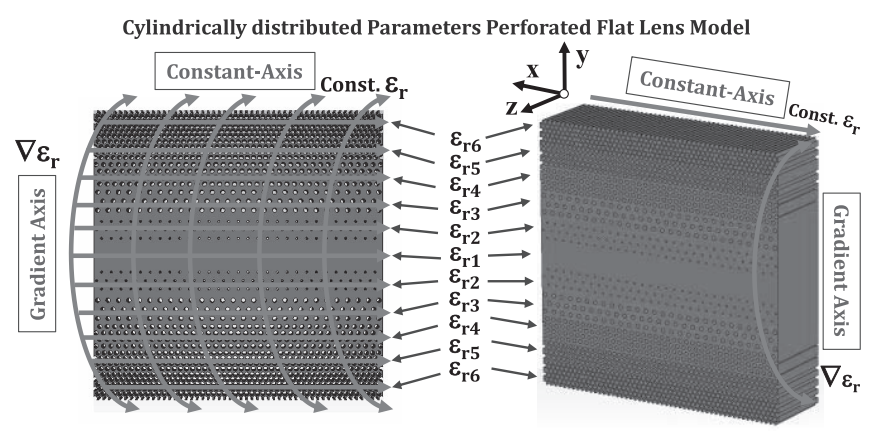

Fig. 2. Cyllindrically distributed parameters flat lens concept and modeling by using triangular unit cells of perforations.

is moved along $y$-or $x$-directions (see Fig. 1), the different permittivity values of the lens produce a linear phase slope that steers the beam, accordingly [11]. Given the lens circular effective parameters distribution, the described behavior is independent of the axis in which the feeding antenna is moved along; the beam will be steered in the same manner.

2) Cylindrically Distributed Parameters Flat Lens Concept: As it has been stated before, a fan-beam radiation pattern (i.e., a beam with a narrow beamwidth in one dimension, broader in the orthogonal) could be very useful for many applications. More specifically, it has been successfully evaluated for high-speed indoor communication systems operating in the $60 \mathrm{GHz}$ band [17], recommending its utilization in certain situations at APs or portable stations (PSs), for example, due to its good immunity to azimuth pointing deviation [17]. Therefore, in order to achieve a fan-beam pattern, a cylindrical lens, to correct the phase of a feeding antenna only in one dimension, in which the beam will be narrower, is needed.

Therefore, in order to achieve a fan-beam radiation pattern, a cylindrical lens, to correct the phase of a feeding antenna only in one dimension, in which the beam will be narrower, is needed. However, it is essential to preserve a planar structure, despite a cylindrical permittivity profile is needed. Hence, the cylindrically distributed parameters lens functioning principle, along its gradient-index axis, is the same as for the previous circular lens described in [11], while in the constantindex axis, the lens is not performing any phase correction, and thus the radiation beam from the source is not being modified. The introduced novel lens achieves the desired behavior at the same time it preserves a planar antenna structure, very interesting for all aforementioned reasons related to APs and PSs.

The cylindrically distributed parameters flat lens concept is depicted in Fig. 2. Fundamentally, consists in a set of eleven rectangular sections of six different permittivity materials, to produce the desired phase delays required to obtain a plane wave, when the lens is illuminated from its central focus position, in the same way as it has been described for the circular lens. Likewise, when the feeding position is moved along $y$-direction (see Fig. 2), the different permittivity values produce a linear phase slope, which steers the beam only along the gradient-index axis (i.e., along $y$-direction), accordingly. As a result of the lens cylindrical parameters distribution,
TABLE I

LTCC PERforated LENSES ChARACTERISTIC PARAMETERS

\begin{tabular}{|c|c|c|c|c|c|}
\hline Section/Ring & $\begin{array}{c}\text { Section/ } \\
\text { Ring thickness }\end{array}$ & $\boldsymbol{\varepsilon}_{\text {reff }}$ & $\boldsymbol{\alpha}$ & $\boldsymbol{d}$ & $\boldsymbol{s}$ \\
\hline$\varepsilon_{\mathrm{r} 1}$ & $2.27 \mathrm{~mm}$ & 7.1 & - & - & - \\
\hline$\varepsilon_{\mathrm{r} 2}$ & $2.27 \mathrm{~mm}$ & 6.79 & 0.051 & $0.2 \mathrm{~mm}$ & $0.845 \mathrm{~mm}$ \\
\hline$\varepsilon_{\mathrm{r} 3}$ & $2.27 \mathrm{~mm}$ & 6.01 & 0.179 & $0.4 \mathrm{~mm}$ & $0.901 \mathrm{~mm}$ \\
\hline$\varepsilon_{\mathrm{r} 4}$ & $2.27 \mathrm{~mm}$ & 4.99 & 0.346 & $0.4 \mathrm{~mm}$ & $0.648 \mathrm{~mm}$ \\
\hline$\varepsilon_{\mathrm{r} 5}$ & $2.27 \mathrm{~mm}$ & 3.92 & 0.521 & $0.4 \mathrm{~mm}$ & $0.528 \mathrm{~mm}$ \\
\hline$\varepsilon_{\mathrm{r} 6}$ & $2.27 \mathrm{~mm}$ & 2.9 & 0.639 & $0.4 \mathrm{~mm}$ & $0.476 \mathrm{~mm}$ \\
\hline
\end{tabular}

if the position of the feeding element is moved along the constant-index axis (i.e., $x$-direction), the beam is maintained invariant, because the phase is not being corrected in this specific dimension, to finally obtain the desired fan-beam pattern.

\section{B. Practical Dielectric Gradient-Index Flat Lens Design}

After an optimization process, with a tradeoff between the maximum achievable gain and aperture dimensions (gain values greater than $14 \mathrm{~dB}$, or even $20 \mathrm{~dB}$, are required to ensure acceptable system performance and range around $60 \mathrm{GHz}$ band [8], [10]), the theoretical lens total dimensions are fixed in $25 \mathrm{~mm} \times 25 \mathrm{~mm}\left(5 \lambda_{60 \mathrm{GHz}} \times 5 \lambda_{60 \mathrm{GHz}}\right)$, and $25 \mathrm{~mm}$ in diameter, for the cylindrically and circularly distributed parameters lenses, respectively, with $7 \mathrm{~mm}$ thickness $\left(1.4 \lambda_{60} \mathrm{GHz}\right)$, and a focal length of $F=6.25 \mathrm{~mm}\left(1.25 \lambda_{60} \mathrm{GHz}\right)$, for both lenses.

Applying the functioning principle and design procedure described in [11] for the circular flat lens, and the same principle for the cylindrically distributed parameters flat lens but considering the particularities explained in the previous section, the set of six different permittivity values needed, respectively, for the different six rings or eleven zones of both lenses are obtained and summarized in Table I.

Then, we selected the DuPont 9k7 $\left(\varepsilon_{r}=7 . l, \tan \delta=\right.$ $0.0009)$ dielectric material in order to model, simulate, and fabricate the final LTCC lens prototypes, using an interesting alternative to traditional fabrication methods, which consists in perforating a single layer of dielectric substrate, as it is described in [11]-[14], to reduce its effective dielectric constant. If the diameter of the holes perforated in the substrate $(d)$ and the distance between them $(s)$ are kept smaller than $\lambda_{\text {eff }} / 2$, the substrate will appear to have a uniform effective permittivity. Hence, the set of characteristic parameters ( $\varepsilon_{\text {reff }}, \alpha, d$, and $s$ ) of the final prototypes modeled by perforations, using triangular unit cells of holes, are also summarized in Table I, where the filling factor $(\alpha)$ is the fraction of area (or volume) of substrate material removed by the perforations to smoothly lower the permittivity from 7.1 to 2.9 , depending on the diameter $(d)$ and distance $(s)$. The complete mathematical expressions to obtain the set of the characteristic parameters, which define the perforated lens, can be found in [12]. 


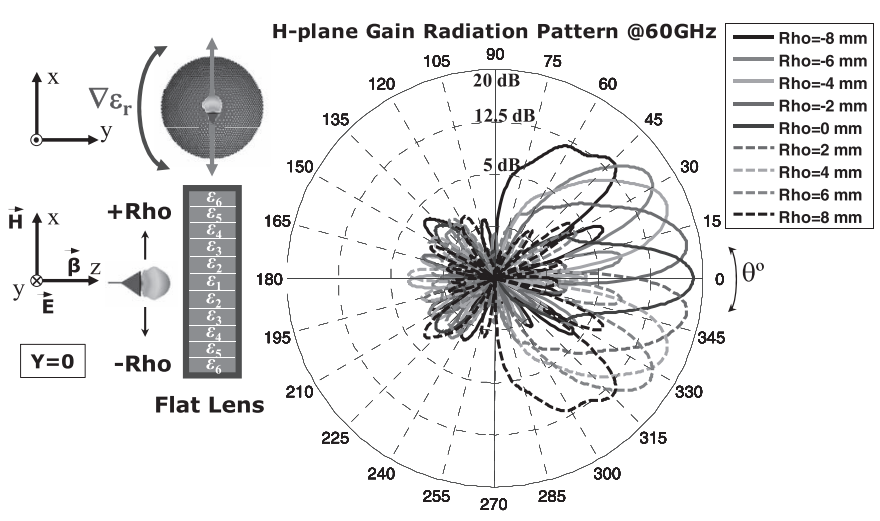

Fig. 3. H-plane gain radiation pattern simulation results at $60 \mathrm{GHz}$ for each Rho position of the WR-15 along $x$-dimension of the circular LTCC lens.

\section{Simulation Results}

In this section, the two designed dielectric flat lenses are briefly numerically analyzed to test their focusing capabilities and performance in the whole frequency band of interest.

1) Circular LTCC Flat Lens Simulation Results: The circular perforated flat lens model has been simulated at $60 \mathrm{GHz}$ band, from 57 to $66 \mathrm{GHz}$, using the time-domain solver of CST Microwave Studio. A complete set of nine different simulations have been performed corresponding to different discrete positions of a radiating element (which could correspond to the positions of antenna elements in a switched-beam array) along $x$-direction (see Fig. 3), going from Rho $=-8 \mathrm{~mm}$ to Rho $=+8 \mathrm{~mm}$, in steps of $2 \mathrm{~mm}$, testing the gain performance and beam-steering capabilities of the lens. The radiating element used consists of a rectangular aperture, a WR-15 openended waveguide model, with the $E$-field linearly polarized along the $y$-direction, which provides an efficient illumination of the lens with around $-14 \mathrm{~dB}$ edge taper in the $\mathrm{H}$-plane. The WR15 model is well matched $\left(S_{11}<-10 \mathrm{~dB}\right)$ in the whole frequency band and for all the feeding positions. The WR-15 open-ended waveguide has been chosen to feed the lenses during the simulations and measurements because it represents a standard very well-known topology for antennas, instead of using other antenna alternatives, despite this would lead to a volumetric antenna configuration. However, a completely planar antenna architecture suitable for mobile devices can be achieved, for example, with the lenses illuminated by a planar array of CPW-fed slot antennas, instead of an openended waveguide, as it is demonstrated in [9]. Nevertheless, in [9] it is shown that the lens performance in terms of gain radiation patterns is comparable to the performance achieved when the lens are fed by an open-ended waveguide. Moreover, the WR-15 feeding offers more flexibility in the setup during the experimental part of this paper. Then, for each Rho position of the feeding waveguide, the corresponding H-plane radiation patters are plotted at $60 \mathrm{GHz}$ in Fig. 3. The simulation results at $60 \mathrm{GHz}$ indicate that with the proposed design we are able to achieve up to $18.6 \mathrm{~dB}$ of broadside gain, beam-steering capabilities in both planes from $-25^{\circ}$ to $+25^{\circ}$ with around $17 \mathrm{~dB}$ gain, and up to $\pm 45^{\circ}$ with around $14 \mathrm{~dB}$ gain, with low sidelobe levels (SLLs). Note that given the lens symmetry

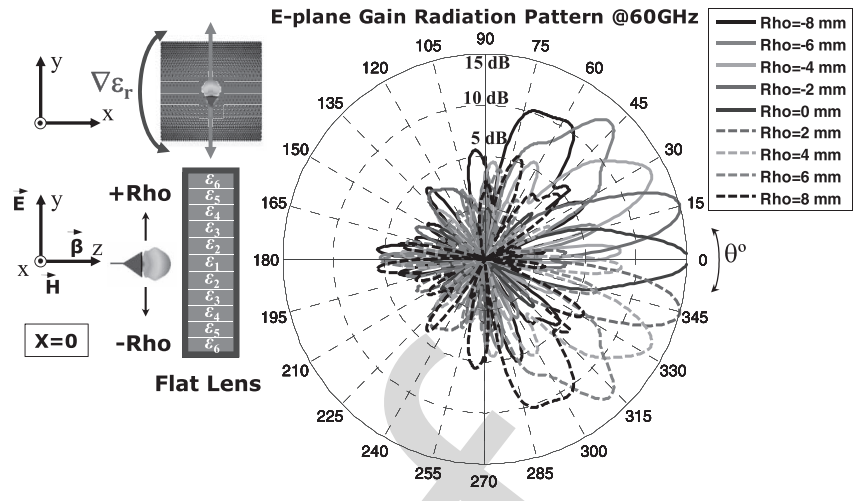

Fig. 4. E-plane gain radiation pattern simulation results at $60 \mathrm{GHz}$ for each Rho feeding position of the WR-15 waveguide along gradient axis of the lens.

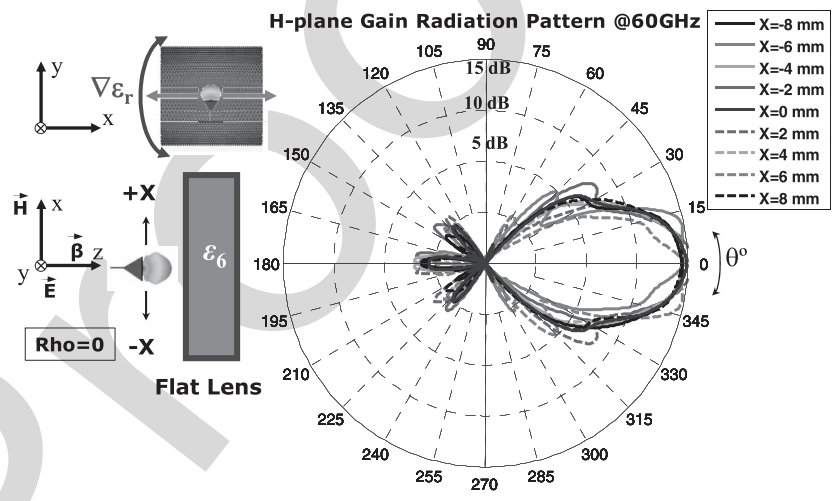

Fig. 5. H-plane gain radiation pattern simulation results at $60 \mathrm{GHz}$ for each $X$ feeding position of the WR-15 waveguide along constant axis of the lens.

identical E-plane radiation patterns are obtained when the lens is fed in the same way as for the H-plane, and therefore are not shown. Moreover, very good gain stability within the whole $60 \mathrm{GHz}$ band is observed from the simulated bandwidth performance, plotted in Fig. 6.

2) Cylindrical LTCC Flat Lens Simulation Results: The cylindrically distributed parameters perforated flat lens model has been simulated from 57 to $66 \mathrm{GHz}$, using the time-domain solver of CST Microwave Studio, in the same way as in the previous section, along the gradient-index axis (to test its beam-steering capabilities), and along the constant-index axis (to test that the beam produced by the lens remains almost invariant despite the position of the feeding antenna in this specific dimension). Therefore, a complete set of nine different simulations have been performed corresponding to different discrete positions of a radiating element along the gradient-index axis (i.e., $y$-direction in Fig. 2), going from Rho $=-8 \mathrm{~mm}$ to Rho $=+8 \mathrm{~mm}$, in steps of $2 \mathrm{~mm}$, testing the beam-steering capabilities of the lens. Another set of nine different simulations have also been performed moving the radiating element along the constant-index axis (i.e., $x$-direction), to test that the beam produced by the lens remains almost invariant despite the position of the feeding antenna.

In both sets of simulations, the radiating element used is a rectangular aperture model, a WR15 waveguide $\left(S_{11}<-10 \mathrm{~dB}\right)$ in the whole frequency band), which provides 


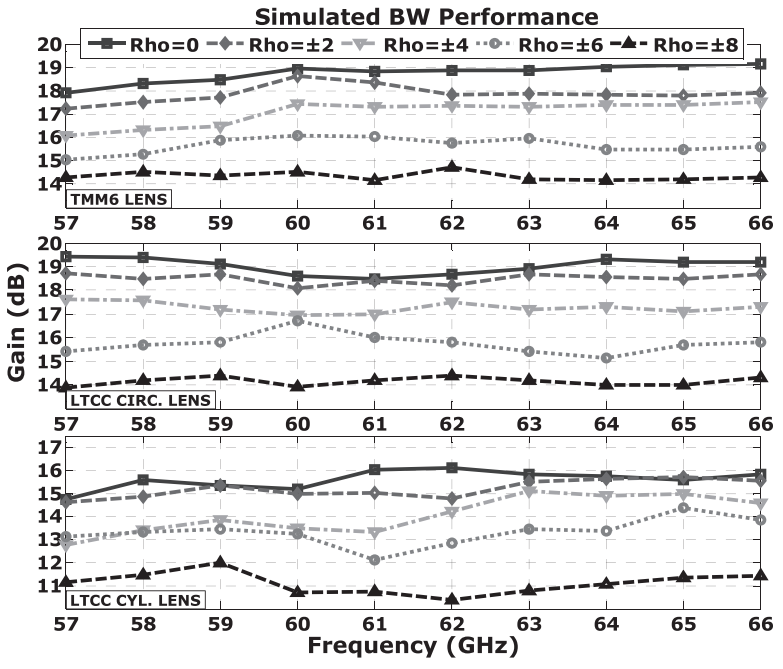

Fig. 6. Simulated bandwith performance: gain for different Rho positions of the WR15 feeding the designed lenses in the whole frequency band of interest. an efficient lens illumination with around $-12 \mathrm{~dB}$ edge taper in the E-plane. Then, for each position of the radiating waveguide, the corresponding E-plane and $\mathrm{H}$-plane radiation patterns are plotted at $60 \mathrm{GHz}$ in Figs. 4 and 5, for the gradient-index and constant-index cases, respectively. As it is shown, the expected behavior of the lens is obtained for both described cases: a radiation beam with around $15 \mathrm{~dB}$ of gain can be steered $\pm 15^{\circ}$ in the gradient axis, and up to $\pm 60^{\circ}$ with more than $10 \mathrm{~dB}$ gain, while a radiation beam with around $15 \mathrm{~dB}$ gain is practically maintained invariant pointing to the broadside direction despite the feeding aperture is being moved along the constant-index axis, allowing us to perform the beam scanning in this direction by using a different technique. The maximum gain obtained in our numerical results is slightly lower compared to the gain achieved with the inhomogeneous circular lenses, because in this case the cylindrically distributed parameters flat lens is performing the phase correction only in one single dimension instead of two. For this reason, the radiation beam obtained is a fan-beam type pattern (i.e., a beam with a narrow beamwidth in one dimension, broader in the orthogonal), which could be also very interesting for some particular applications such as radar and imaging systems, and more specifically for high-speed indoor communication systems at $60 \mathrm{GHz}$, in which this kind of pattern has been successfully assessed [17]. From the simulation results, we also obtain total and radiation efficiencies around $90 \%-95 \%$ for the lens fed with the aforementioned rectangular aperture, since a low-loss LTCC substrate is used.

3) Rogers TMM6 Flat Lens Simulation Results: For comparison purposes, the circular dielectric flat lens introduced in [11] is also considered during the experimental assessments carried out along this paper. Since the radiation pattern numerical results obtained for this lens have been already published in [11], they are not shown here. Instead, the bandwidth performance for the circular TMM6 lens, and for the two new LTCC lenses, is plotted in Fig. 6.

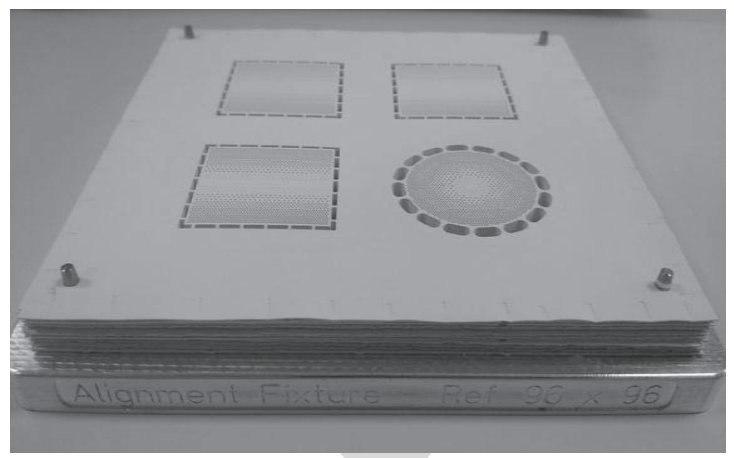

Fig. 7. LTCC dielectric flat lens prototypes fabrication: 31 DuPont 9k7 layers aligned and stacked together before the lamination process.

\section{LTCC FABRICATION OF THE PROTOTYPES}

Once the new designed LTCC lenses have been numerically tested, and promising simulation results were obtained, different prototypes have been fabricated at the facilities of the Universitat Politècnica de València in LTCC technology in order to, first, characterize their performance with a complete set of measurements, and then, experimentally evaluate their practical application as smart antenna solution for high data rate $5 \mathrm{G}$ millimeter-wave systems. A good description of the complete LTCC fabrication process can be found in [19]. Essentially, the LTCC process consists in building a multilayered substrate structure with the capability of printing different metallization individually in each single dielectric glass/ceramic sheet (called green tape). Thus, LTCC allows processing all the design layers separately.

Once all the layers are processed in parallel, separately, they are stacked, laminated together at high pressure in an isostatic process (around $210 \mathrm{~kg} / \mathrm{cm}^{2}$ ), and co-fired (sintering process) at a temperature of $850{ }^{\circ} \mathrm{C}$ during $26.5 \mathrm{~h}$. After a preconditioning process, in which each sheet of smooth green-tape dielectric substrate is heated up to $120{ }^{\circ} \mathrm{C}$ during $20 \mathrm{~min}$, we perform at each layer a total of around 1500 holes with a via punching process machine, to finally achieve the desired gradient-index permittivity profile in one axis, while a constant-index profile is achieved in the orthogonal one, for the cylindrically distributed lens, and a gradient index along both axis, for the circular lens. These small holes, of only 0.4 and $0.2 \mathrm{~mm}$ in diameter, are performed on the soft $254 \mu \mathrm{m}$ thickness DuPont GreenTape 9k7 dielectric substrate. After the punching process, the 31 layers needed to finally build the lens are stacked together, laminated, and sintered in order to obtain a single monolithic structure of $7 \mathrm{~mm}$ thickness. During the lamination and sintering LTCC processes, the material is shrinking $11.8 \%$ in $z$-direction and $9.1 \%$ in $x$ - and $y$-directions, and therefore, we previously considered this shrinkage of the substrate material before manufacturing the final lens design to achieve the characteristic parameters explained in Section II (lens thickness, via-hole dimensions, and separation between holes).

It is remarkable that the proposed fabrication method reduces considerably the final fabrication time compared to the fabrication time needed for manufacturing the TMM6 lens 


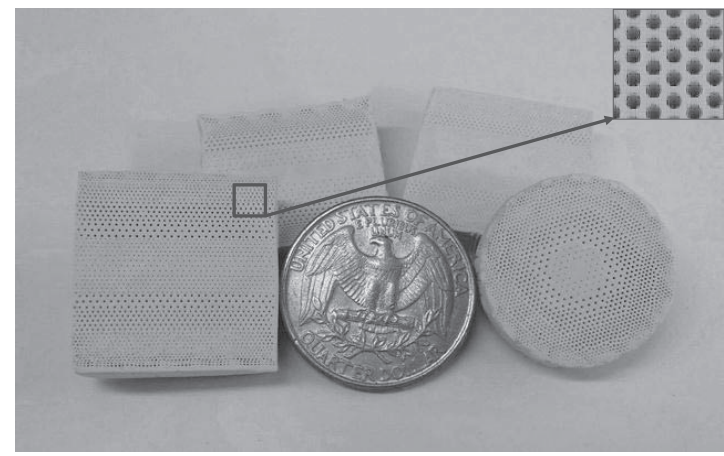

Fig. 8. LTCC dielectric flat lens prototypes with the effective permittivity circularly and cylindrically distributed. A microscopic image of a high hole density zone is shown in the inset of the upper-right corner.

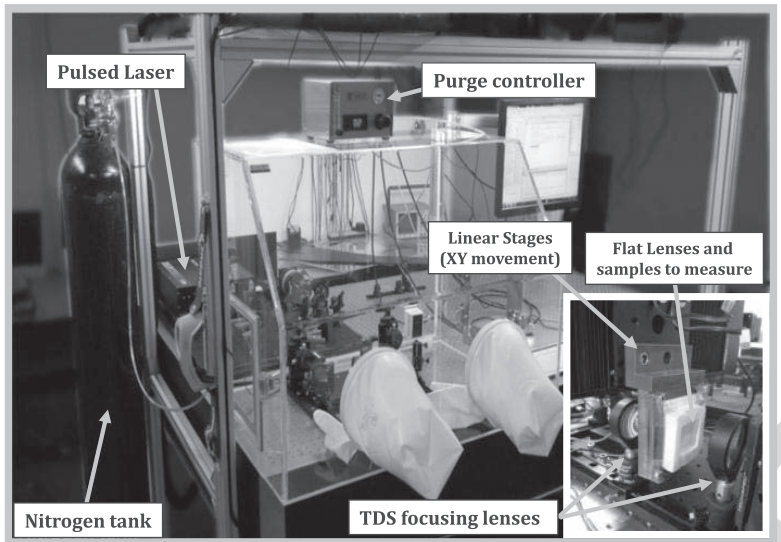

Fig. 9. TDS system placed on an optical talbe used to characterize different materials. A detailed image of the two focusing lenses of the system and the lens under test placed in between is shown in the inset.

introduced in [11], which was huge using carbide drills on a hard substrate, because the LTCC process allows to perform 1000 holes/min on each soft substrate layer. A photograph of the set of 31 DuPont LTCC material layers stacked to build the lenses is shown in Fig. 7, and a photograph of final prototypes is shown in Fig. 8, where a detailed microscopic image of a high-density zone of holes is additionally provided.

\section{Flat Lenses Measurement Results}

A set of measurements have been conducted at AntennaLab facilities of the Universitat Politècnica de Catalunya in order to characterize the performance of the introduced flat lenses for future high-speed $5 \mathrm{G}$ millimeter-wave applications.

\section{A. Flat Lenses Permittivity Profile Measurements}

Before testing the performance of the two dielectric flat lenses in terms of radiation patterns parameters, S-parameters, or efficiencies, it is fundamental and very interesting to assert that the required permittivity profiles have been achieved after the LTCC fabrication process.

With the described purpose, to precisely measure the permittivity profile of the fabricated prototypes a time-domain spectroscopy (TDS) system has been used. Our complete TDS measurement system is shown in Fig. 9. It consists of a femtosecond pulsed laser, which generates very short pulses that are sampled by using an optical delay stage. Once the complete

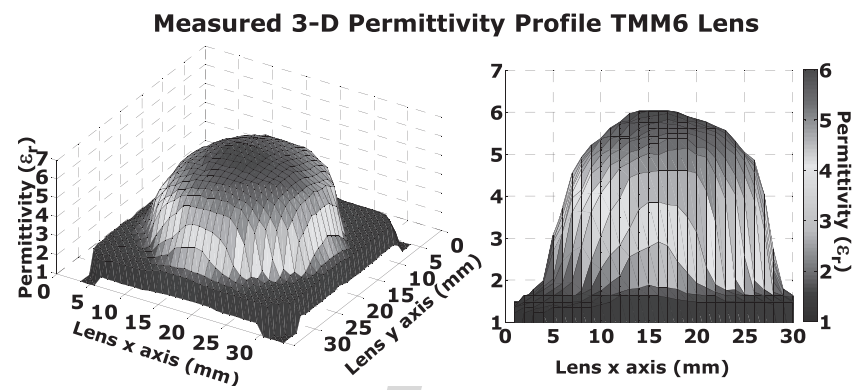

Measured 3-D Permittivity Profile LTCC Circular Lens

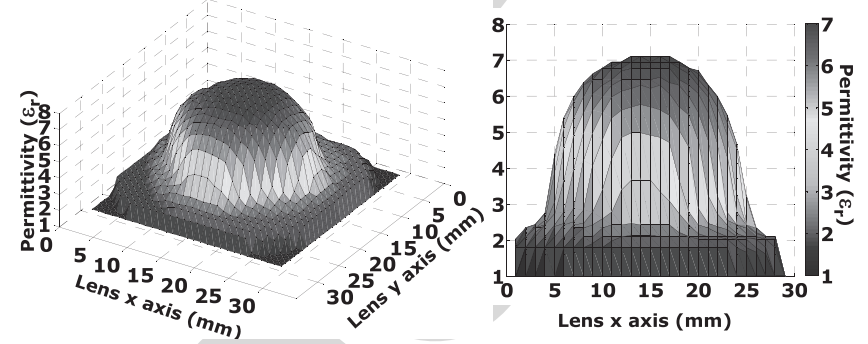

Measured 3-D Permittivity Profile LTCC Cylindrical Lens

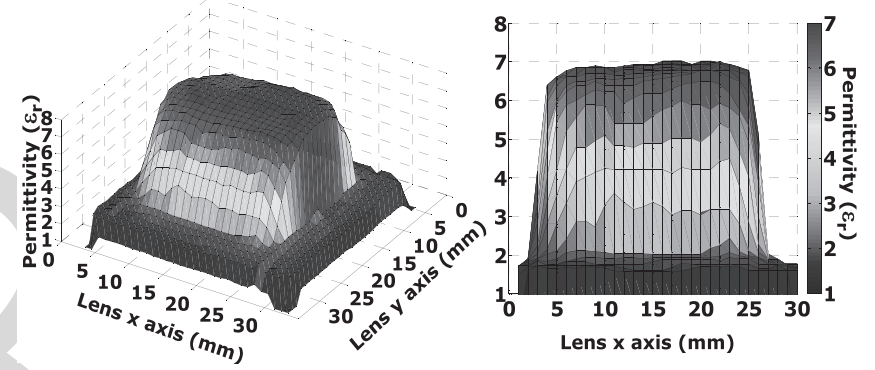

Fig. 10. 3-D representation of the mesured permittivity profile for the circular TMM6 lens (top), LTCC circular (middle), and LTCC cylindrical (bottom).

pulse is retrieved, a discrete Fourier transform is performed in order to obtain the spectrum, as it is usually realized in most of the TDS systems. In this specific case, despite our TDS system is a terahertz-TDS system, which is able to measure up to $1-1.5 \mathrm{THz}$, it is also capable of measuring with a dynamic range (DR) above $30 \mathrm{~dB}$ around $60 \mathrm{GHz}$, and with a DR above $50 \mathrm{~dB}$ around $100 \mathrm{GHz}$. Taking advantage of the small beam spot generated by our TDS system, which is collimated with two focusing lenses placed after the photoconductive receiver and transmitter antennas, we are able to precisely characterize the permittivity of different materials by using the delay produced introducing the sample in between, compared to the signal in free space. First, a solid sample of the DuPont 9k7 LTCC material has been measured, validating the maximum permittivity around 7 , as it was expected. After that, some different samples with uniform hole distribution have also been tested, obtaining the expected results as well, confirming the anticipated behavior.

Therefore, in order to measure the complete permittivity profile over the whole flat lens surface, the prototype is placed in between the two focusing lenses of the TDS system. With the help of two linear stages (to perform the specific movement needed in the $x$ - and $y$-axes), the TDS narrow radiation beam is scanned in steps of $1 \mathrm{~mm}\left(\lambda_{060 \mathrm{GHz}} / 5\right)$ over the lens surface. 


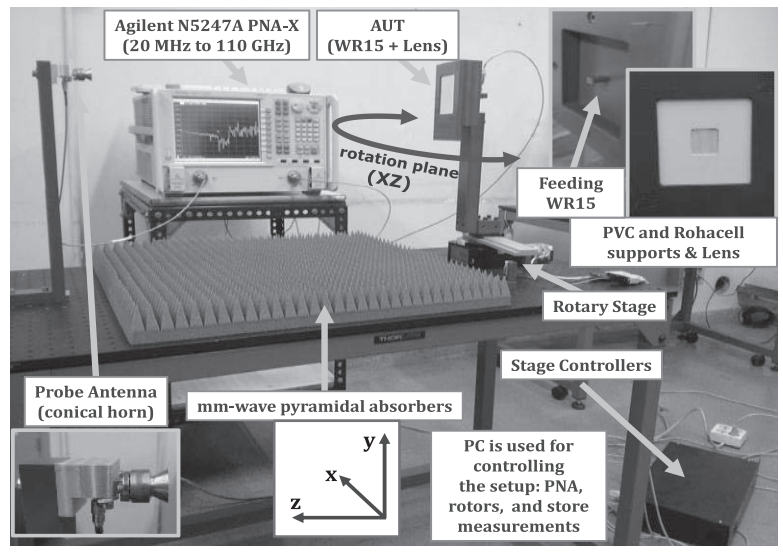

Fig. 11. Far-field radiation pattern measurement setup at $60 \mathrm{GHz}$ band. Detailed images of the WR-15 and lens on PVC supports are shown in the insets.

The 3-D representations of the measured permittivity profiles for the circular TMM6, and for the circular and cylindrical LTCC lenses, are plotted in Fig. 10. As it is shown, despite the physical shape of the designed lenses, with an absolutely planar structure, the permittivity profile is very well defined in all the cases for all the considered lenses, thus demonstrating the good fabrication results, confirming the viability in LTCC fabrication process.

\section{B. Flat Lenses Performance Evaluation}

A complete set of electromagnetic performance measurements for all the designed flat lenses has been carried out in the AntennaLab facilities of the UPC.

1) Radiation Pattern Measurement Results: The far-field radiation patterns produced by all the considered lenses fed with a WR-15 open-ended waveguide have been measured from 57 to $66 \mathrm{GHz}$ using the measurement setup shown in Fig. 11. It is composed of an Agilent N5247A vector network analyzer, a precision rotary stage to perform the scanning of the antennas under test (AUT) in the $x z$ plane (see Fig. 11), stage controllers, a WR-15 waveguide to feed the lens, a conical horn antenna used as a probe, some RF absorbers in order to avoid undesired reflections between the instrumentations, and a computer for controlling the automatization of the complete setup.

A total of nine measurements have been performed for the circular LTCC lens corresponding to different Rho feeding positions of the transmitting WR-15 waveguide (going from Rho $=-8 \mathrm{~mm}$ to Rho $=+8 \mathrm{~mm}$ ) in steps of $2 \mathrm{~mm}$ along the $x$-direction, with the waveguide linearly polarized in the $y$-direction, as it is depicted in the scheme of Fig. 12. Once the radiation patterns are measured, in order to obtain the gain radiation patterns, the AUT is replaced for a wellknown conical horn antenna (used as a reference) to perform a power level comparison. Therefore, the corresponding H-plane gain radiation pattern results are plotted in Fig. 12 at $60 \mathrm{GHz}$. In general, very good agreement is observed between simulation results (Fig. 3) and measurements. In the broadside direction we achieve up to $17.5 \mathrm{~dB}$ gain, with

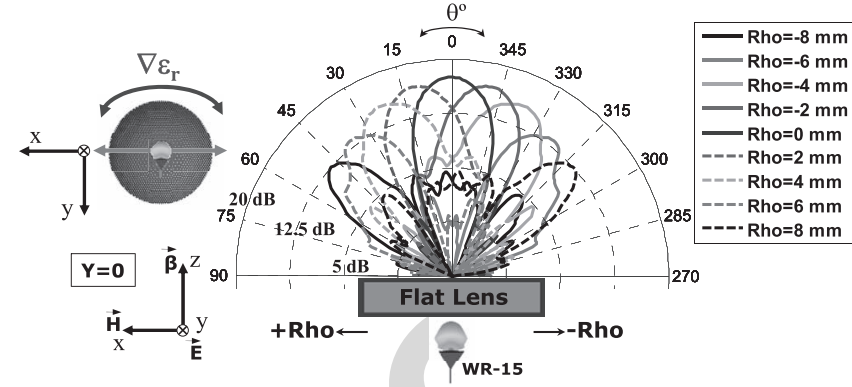

Fig. 12. Complete set of measured $\mathrm{H}$-plane gain radiation patterns at $60 \mathrm{GHz}$ for each Rho feeding position of the WR-15, for the circular LTCC lens.

TABLE II

SUMMARY OF TMM6 AND LTCC CIRCULAR LENSES PERFORMANCE AT $60 \mathrm{GHz}$ (H-Plane PARAMETERS)

\begin{tabular}{|c|c|c|c|c|c|c|c|c|}
\hline \multirow{2}{*}{ Rho } & \multicolumn{4}{|c|}{ TMM6 Circular Lens } & \multicolumn{4}{c|}{ LTCC Circular Lens } \\
\cline { 2 - 9 } & Gain & $\left(\boldsymbol{\theta}^{\circ}\right) \mathbf{s c a n}$ & $\boldsymbol{\Delta} \boldsymbol{\theta}_{-\mathbf{3 B}}$ & SLL & Gain & $\left(\boldsymbol{\theta}^{\circ}\right) \mathbf{s c a n}$ & $\boldsymbol{\Delta} \boldsymbol{\theta}_{-\mathbf{3 d B}}$ & SLL \\
\hline $0 \mathrm{~mm}$ & $18.3 \mathrm{~dB}$ & $0^{\circ}$ & $14^{\circ}$ & $-18 \mathrm{~dB}$ & $17.5 \mathrm{~dB}$ & $0^{\circ}$ & $21^{\circ}$ & $-15.8 \mathrm{~dB}$ \\
\hline $\pm 2 \mathrm{~mm}$ & $17.2 \mathrm{~dB}$ & $\pm 10^{\circ}$ & $15.1^{\circ}$ & $-13 \mathrm{~dB}$ & $16.7 \mathrm{~dB}$ & $\pm 12^{\circ}$ & $22^{\circ}$ & $-12 \mathrm{~dB}$ \\
\hline $\pm 4 \mathrm{~mm}$ & $16.6 \mathrm{~dB}$ & $\pm 22^{\circ}$ & $16.7^{\circ}$ & $-11.2 \mathrm{~dB}$ & $15.1 \mathrm{~dB}$ & $\pm 23^{\circ}$ & $23^{\circ}$ & $-8.9 \mathrm{~dB}$ \\
\hline $\pm 6 \mathrm{~mm}$ & $14.7 \mathrm{~dB}$ & $\pm 32^{\circ}$ & $17.8^{\circ}$ & $-10.5 \mathrm{~dB}$ & $12.9 \mathrm{~dB}$ & $\pm 37^{\circ}$ & $20^{\circ}$ & $-12.2 \mathrm{~dB}$ \\
\hline $\pm 8 \mathrm{~mm}$ & $13.7 \mathrm{~dB}$ & $\pm 48^{\circ}$ & $21^{\circ}$ & $-7.8 \mathrm{~dB}$ & $11.2 \mathrm{~dB}$ & $\pm 48^{\circ}$ & $17^{\circ}$ & $-7.8 \mathrm{~dB}$ \\
\hline
\end{tabular}

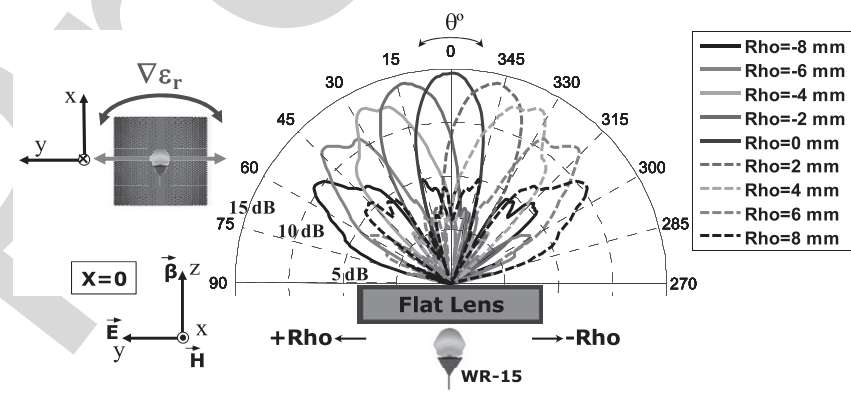

Fig. 13. Measured E-plane gain radiation patterns at $60 \mathrm{GHz}$ for each Rho feeding position of the WR-15 waveguide along gradient axis of the lens.

beam-steering capabilities from $-25^{\circ}$ to $+25^{\circ}$ with around $15 \mathrm{~dB}$, and up to $\pm 45^{\circ}$ with more than $11 \mathrm{~dB}$ gain. Additionally, the most important radiation pattern parameters at $60 \mathrm{GHz}$ are summarized in Table II, in order to concisely compact all the interesting and most relevant measurement results for a better analysis in the next experimental section in which the practical use of the three considered lenses as smart antenna systems is evaluated.

In the same way, a total of nine measurements have been performed for the cylindrical LTCC flat along the gradientindex axis of the lens, and nine additional measurements along the constant-index axis, in order to obtain the gain radiation patterns produced by the lens when is fed by a WR-15 waveguide. Therefore, the corresponding E-plane gain radiation patterns results are plotted in Fig. 13 at $60 \mathrm{GHz}$ (WR-15 with the electric field $y$-direction polarized, as it is depicted).

As it is observed, as the Rho feeding position is moved leftwards, the high-gain radiation pattern produced by the lens is steered rightwards (and vice versa), accordingly. Compared to the simulation results (Fig. 4), in general there is a very good agreement. $\mathrm{Up}$ to $14.8 \mathrm{~dB}$ gain in the broadside 


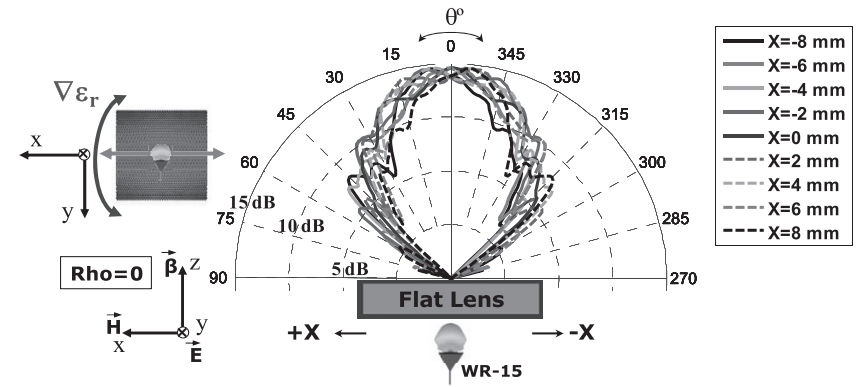

Fig. 14. Measured H-plane gain radiation patterns at $60 \mathrm{GHz}$ for each $X$ feeding position of the WR-15 waveguide along constant axis of the lens.

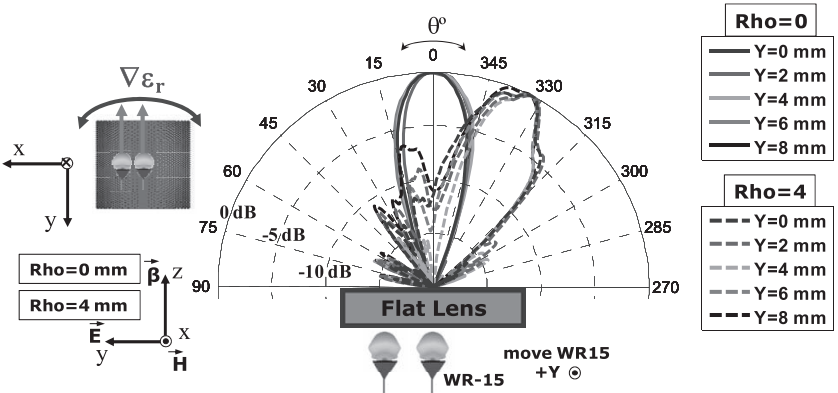

Fig. 15. Measured normalized E-plane radiation patterns at $60 \mathrm{GHz}$ (cut along gradient-index axis) moving the WR-15 in the constant axis of the lens (along $X$ ) for Rho $=0 \mathrm{~mm}$ and $\mathrm{Rho}=4 \mathrm{~mm}$ feeding positions. direction is achieved, with around $13 \mathrm{~dB}$ at $\pm 25^{\circ}$, and more than $10 \mathrm{~dB}$ when the beam is scanned $\pm 55^{\circ}$. In addition, the corresponding nine H-plane gain radiation patterns for nine different positions of the WR-15 waveguide along the constant-index axis of the lens (i.e., $x$-direction), maintaining the Rho position centered to the lens (Rho $=0 \mathrm{~mm}$ ), are plotted in Fig. 14. As it is shown, despite moving the feeding aperture, the beams are maintained almost invariant in this specific dimension, as it was expected.

Moreover, going one step further in this sense, the E-plane radiation patterns corresponding at different $X$ feeding positions of the WR-15 along the constant-index axis of the lens, keeping invariant the Rho position, are plotted in Fig. 15. Only the Rho $=0 \mathrm{~mm}(X=0-8 \mathrm{~mm})$ and Rho $=4 \mathrm{~mm}$ $(X=0-8 \mathrm{~mm})$ feeding positions are plotted in order to avoid cluttering the figure, but it is enough to observe and confirm the previously described behavior, which is also obtained for the rest of the feeding positions.

As it is noticed, although the WR-15 is moved in the $x$-dimension, the E-plane (vertical cut, $y$-direction in Fig. 15), is maintained practically invariant for all $X$ positions of the open-ended waveguide. As for the circular LTCC lens, the most important radiation pattern parameters at $60 \mathrm{GHz}$ are summarized in Table II for the cylindrical LTCC lens. Moreover, the same set of radiation patterns measurements are also carried out for the TMM6 lens introduced in [11] with our setup. Then, for comparison purposes and due to the fact that this lens is also evaluated in the next experimental section, the most important radiation pattern parameters at $60 \mathrm{GHz}$ are also summarized in Table II. As it is shown, with the circular LTCC lens, we achieve similar radiation pattern

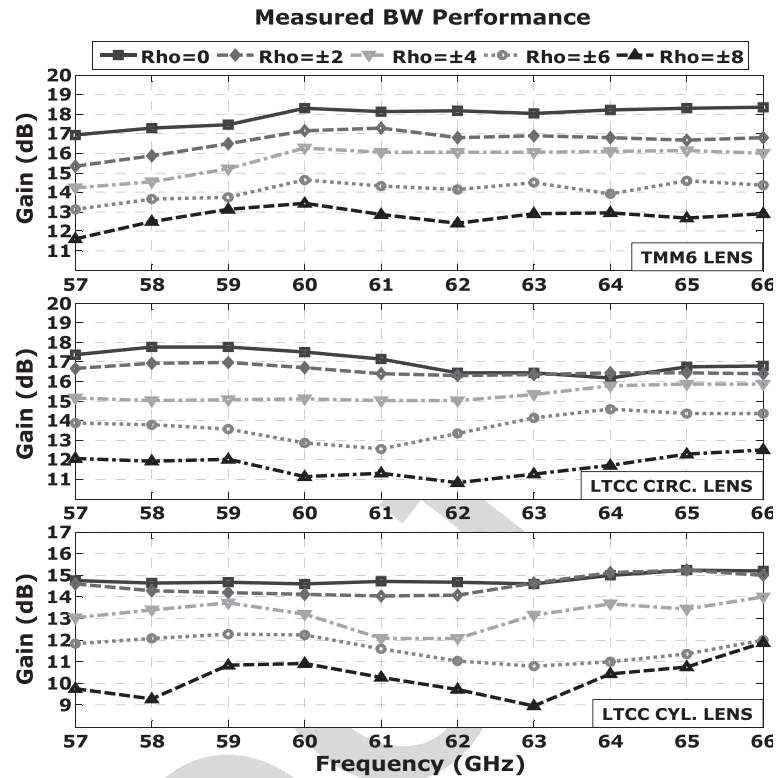

Fig. 16. Measured bandwidth performance: maximum gain for different Rho feeding positions of the WR-15 along gradient-index axis of the lenses.

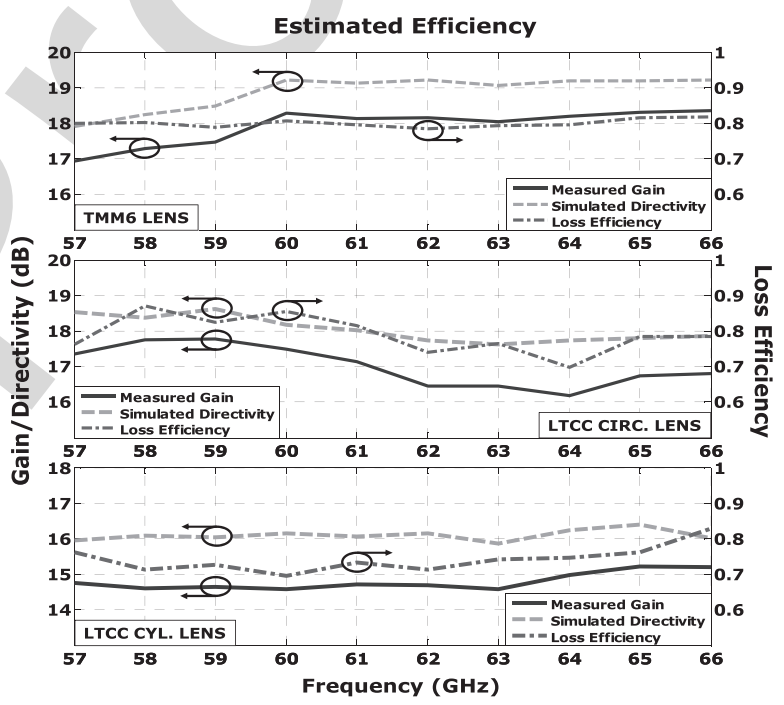

Fig. 17. Estimated loss efficiency computed from simulated directivity and measured gain values for the three considered lenses.

characteristics as with the circular TMM6 lens, with slightly lower gain values at some frequencies and scanning angles, because in this case the highest permittivity value is 7 instead of 6, thus slightly higher reflection is obtained in the dielectricvacuum (free space) transition.

2) Measured Bandwidth Performance: In addition, the gain over the whole $60 \mathrm{GHz}$ frequency band of interest has been measured for all the Rho feeding positions, and it is plotted in Fig. 16, for the three considered dielectric flat lenses. As it shown, very good gain stability is observed for the three lenses, confirming the good broadband behavior obtained in the numerical results. This is a remarkable result because in general it is very difficult to achieve antenna systems with broadband operation behavior.

3) Estimated Efficiencies: The estimated loss efficiencies for the three flat lenses are also reported in Fig. 17, 


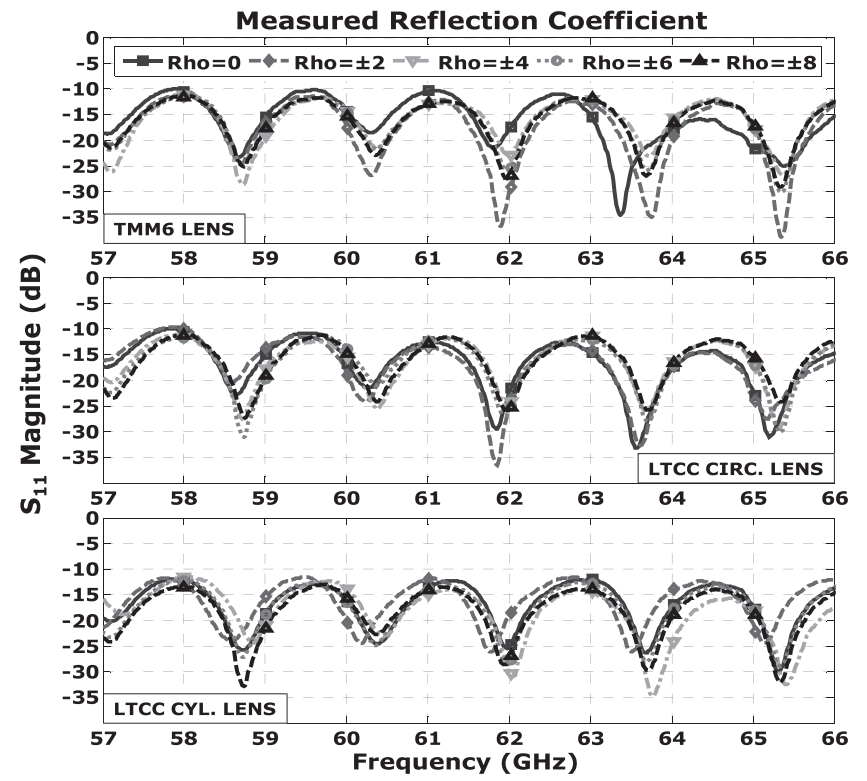

Fig. 18. Measured $S_{11}$ parameter of the three lenses for different Rho feeding positions of the WR-15 waveguide in the whole frequency band of interest.

from 57 to $66 \mathrm{GHz}$, computed from CST simulation results of the directivity and measured gain values, since with our setup we are not able to measure the complete 3-D radiation patterns in order to integrate the whole power to obtain directly the efficiency or the directivity. As it is shown, almost constant values around $70 \%-80 \%$, and above, are estimated in all the cases for the whole frequency band, since low-loss dielectric materials are used to build the lenses.

4) Measured Reflection Coefficient: The measured $S_{11}$ parameters obtained, after applying a short-open-load-thru (SOLT) calibration, for the different flat lenses fed with the corresponding WR-15 open-ended waveguide in the different Rho positions are plotted in Fig. 18, for the whole frequency band. As it is shown, all the measured reflection coefficients are below $-10 \mathrm{~dB}$, as it was expected.

\section{Assessment of the Flat Lens Performance in A REAL $60 \mathrm{GHZ}$ WPAN INDOOR ENVIRONMENT}

Once the three considered dielectric flat lenses have been fully electromagnetically characterized and remarkable good measurement results have been obtained, their performance is experimentally evaluated and compared to a single commercial omni-directional antenna, as well as their use as smart antennas is experimentally compared to a traditional ULA in real $60 \mathrm{GHz}$ WPAN environment.

\section{A. Introduction}

For this experimental part, we have considered an indoor scenario in the facilities of the Universidad Politécnica de Cartagena (UPCT) varying the position of the receiver ( $\mathrm{Rx}$ ) antenna. Three different positions for the $\mathrm{Rx}$ antenna have been measured forming an angle of $0^{\circ}, 22.5^{\circ}$, and $45^{\circ}$ with respect to the transmitting (Tx) antenna, which is placed in a fixed position. The receiver antenna is, in all the cases, a single commercial Q-par QOM55-65 VRA 55 to $65 \mathrm{GHz}$

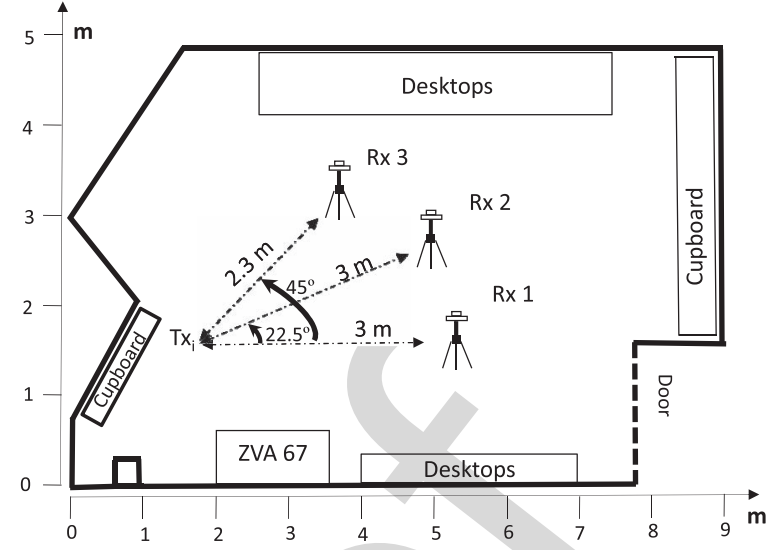

Fig. 19. Indoor scenario and experimental measurement setup arrangement.

omni-directional V-type antenna. The gain of this antenna varies from 4.3 to $5.2 \mathrm{~dB}$ within the considered 57 to $64 \mathrm{GHz}$ frequency band, and the typical $3 \mathrm{~dB}$ elevation beamwidth ranges from $24^{\circ}$ to $33^{\circ}$, while being omni directional in the horizontal plane. In this paper, the considered Tx antennas are the three presented lenses fed by the same rectangular aperture WR-15 waveguide used during the previous sections, the same commercial omni-directional antenna used in the Rx part, and a virtual ULA modeled with ten positions of this same omnidirectional antenna. The performance test with the considered Tx antennas is carried out in direct LOS conditions for all the angles between $\mathrm{Tx}$ and $\mathrm{Rx}$, and also in OLOS conditions for the $0^{\circ}$ case. In the following sections, all the important considerations about the experimental scenario, channel sounder, and methodology are conveniently described before to proceed with the analysis of the measurement results.

\section{B. Experimental Scenario}

The scenario for this experimental study is a laboratory of the UPCT. The laboratory is an almost rectangular room of about $5 \mathrm{~m} \times 9 \mathrm{~m}$ furnished with several closets, desktops, and shelves. The laboratory scheme with the measurement setup arrangement is depicted in Fig. 19. As it is shown in Fig. 19, the three different considered Tx-Rx situations are established as follows: $3 \mathrm{~m}$ between Tx and Rx forming an angle of $0^{\circ}$ (first position), $3 \mathrm{~m}$ between $\mathrm{Tx}$ and $\mathrm{Rx}$ forming an angle of $22.5^{\circ}$ (second position), and at $2.3 \mathrm{~m}$ between $\mathrm{Tx}$ and $\mathrm{Rx}$ forming an angle of $45^{\circ}$ (third position).

\section{Channel Sounder and Methodology}

The channel sounder and the methodology employed in this paper are exactly the same as the followed and exhaustively explained in [20]. A VNA is used to measure the transmission $\left(S_{21}\right)$ parameter in order to obtain in the frequency domain the complex transfer function of a wireless system. The frequency domain function measured $H(f)$ is acquired. Then, the relative received power $(P)$ is computed. This parameter, which is defined as the ratio between the transmitted and the received powers, is important for $5 \mathrm{G}$ communication systems because describes the attenuation of the transmitted 


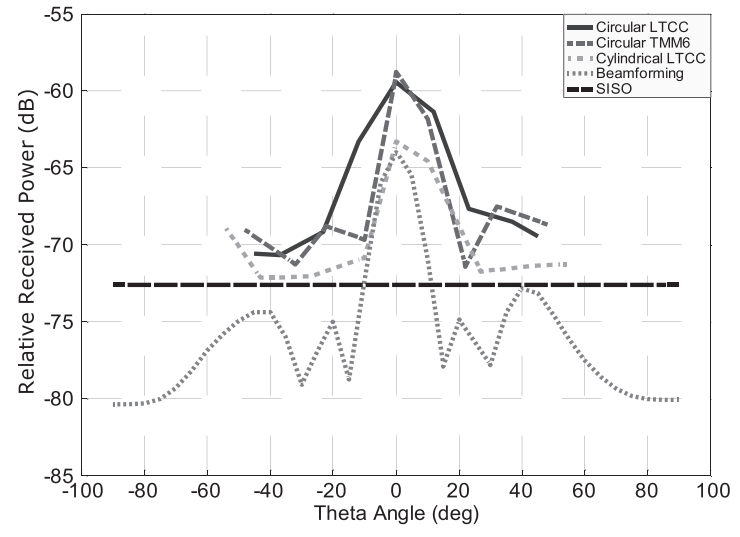

Fig. 20. Relative received power at first position $\left(\theta=0^{\circ}\right)$ in LOS conditions.

TABLE III

Summary of LTCC CYLINDRICAL LENS PERFormanCE AT $60 \mathrm{GHz}$ (E-Plane AND H-Plane PARAMETERS)

\begin{tabular}{|c|c|c|c|c|c|c|c|c|}
\hline \multirow{2}{*}{$\boldsymbol{R} \boldsymbol{h} \boldsymbol{o} \boldsymbol{o}$} & \multicolumn{3}{|c|}{ E-PLANE (along gradient axis) } & \multicolumn{3}{c|}{ H-PLANE (along constant axis) } \\
\cline { 2 - 9 } & Gain & $\left(\boldsymbol{\theta}^{\circ}\right)$ scan & $\boldsymbol{\Delta} \boldsymbol{\theta}_{-3 \mathrm{~dB}}$ & SLL & Gain & $\left.\boldsymbol{\theta}^{\circ}\right)$ scan & $\boldsymbol{\Delta}_{-3 \mathrm{~dB}}$ & SLL \\
\hline $0 \mathrm{~mm}$ & $14.6 \mathrm{~dB}$ & $0^{\circ}$ & $19^{\circ}$ & $-17.7 \mathrm{~dB}$ & $14.6 \mathrm{~dB}$ & $0^{\circ}$ & $48^{\circ}$ & $-17.5 \mathrm{~dB}$ \\
\hline $\pm 2 \mathrm{~mm}$ & $14.1 \mathrm{~dB}$ & $\pm 13^{\circ}$ & $21^{\circ}$ & $-12 \mathrm{~dB}$ & $14.4 \mathrm{~dB}$ & $0^{\circ}$ & $44^{\circ}$ & $-8.9 \mathrm{~dB}$ \\
\hline $\pm 4 \mathrm{~mm}$ & $13.2 \mathrm{~dB}$ & $\pm 27^{\circ}$ & $20^{\circ}$ & $-11.5 \mathrm{~dB}$ & $14.3 \mathrm{~dB}$ & $0^{\circ}$ & $46^{\circ}$ & $-9.6 \mathrm{~dB}$ \\
\hline $\pm 6 \mathrm{~mm}$ & $12.3 \mathrm{~dB}$ & $\pm 43^{\circ}$ & $21^{\circ}$ & $-8.9 \mathrm{~dB}$ & $14.6 \mathrm{~dB}$ & $0^{\circ}$ & $35^{\circ}$ & $-10.6 \mathrm{~dB}$ \\
\hline $\pm 8 \mathrm{~mm}$ & $10.9 \mathrm{~dB}$ & $\pm 54^{\circ}$ & $17^{\circ}$ & $-5.5 \mathrm{~dB}$ & $14.5 \mathrm{~dB}$ & $0^{\circ}$ & $35^{\circ}$ & $-14 \mathrm{~dB}$ \\
\hline
\end{tabular}

radio link in a specific angular direction. Next, the time domain function $h(t)$ is obtained by using the inverse fast Fourier transform. Last, the power delay profile (PDP) and the RMSdelay spread $\left(\sigma_{\tau}\right)$, which represents the standard deviation of the PDP, are calculated, as it is exhaustively explained in [20]. In this case, the RMS delay spread is a fundamental parameter in order to have a notion of the multipath characteristics of a communications channel. The longer the RMS delay spread, the smaller the coherence bandwidth, which directly affects and limits the capacity in a $5 \mathrm{G}$ wireless communication system.

\section{Experimental Results}

In the following sections the experimental results obtained for the three considered positions are reported and compared for the three dielectric flat lenses, and for the ULA and SISO cases.

1) First Position Measurements: As it is depicted in Fig. 19, in the first position situation the Tx and the Rx antennas are separated $3 \mathrm{~m}$ forming an angle of $0^{\circ}$ between them. For the LOS condition, the absorbent panel placed in between the Tx and Rx is removed. Then, the methodology detailed in [20] is applied obtaining the following results for all the considered antennas.

For the LOS situation, the relative received power in function of the angle and the PDP are plotted for each different transmitting antenna in Figs. 20 and 21, respectively.

As it is observed in Fig. 20, the highest relative received power is achieved using the circular TMM6 as a transmitting antenna, as it was expected from the measured radiation pattern parameter results obtained in the previous sections (see Tables II and III). In any case, with all the designed

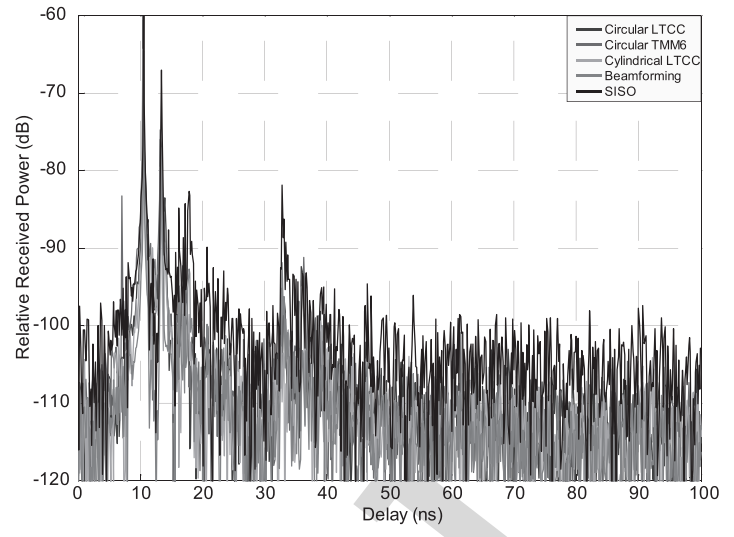

Fig. 21. PDP at first position $\left(\theta=0^{\circ}\right)$ in LOS conditions.

TABLE IV

RELATIVE RECEIVED POWER AND RMS DELAY SPREAD VALUES FOR FIRST POSITION IN LOS CONDITIONS

\begin{tabular}{|c|c|c|}
\cline { 2 - 3 } \multicolumn{1}{c|}{} & Relative Received Power & RMS Delay Spread \\
\hline Circular LTCC & $-59.45 \mathrm{~dB}$ & $0.68 \mathrm{~ns}$ \\
\hline Circular TMM6 & $-58.79 \mathrm{~dB}$ & $0.66 \mathrm{~ns}$ \\
\hline Cylindrical LTCC & $-63.30 \mathrm{~dB}$ & $0.64 \mathrm{~ns}$ \\
\hline 10-elem. ULA & $-63.96 \mathrm{~dB}$ & $1.21 \mathrm{~ns}$ \\
\hline SISO & $-72.56 \mathrm{~dB}$ & $4.47 \mathrm{~ns}$ \\
\hline
\end{tabular}

lenses the relative received power is better compared to using a beamforming technique applied to the ten-element ULA. In Fig. 21, the measured PDP shows that for all cases, direct ray with highest power (LOS component) is received at $10.5 \mathrm{~ns}$. The rest of the components arrive attenuated in the next moments due to the multipath propagation. It is worthwhile mention that the shape obtained for all the PDPs is almost identical, which means that the situation of the antennas has been the same during the whole process of the measurements campaign, fact that is very difficult in this kind of measurements at these frequencies.

In the Table IV, the relative received power and the RMS delay spread calculated from the PDP for each evaluated transmitting antenna are summarized. As it is observed in Table IV, the highest relative received power is achieved with the circular TMM6 lens, which it has been stated before.

Additionally, the power difference among the rest is according to the measured gain values (see Tables II and III). For example, the measured gain difference obtained in previous sections between the circular TMM6 lens and the circular LTCC is around $0.8 \mathrm{~dB}$, which is almost the same relative received power difference obtained for this first measured position in LOS conditions. Similar results are also obtained comparing the TMM6 lens and the cylindrical LTCC lens: a measured gain difference of $3.7 \mathrm{~dB}$ between the two lenses, and a relative received power difference of $4.5 \mathrm{~dB}$. Moreover, a remarkable result is that the measured relative received power for the ten-element ULA is lower than the measured for all the designed lenses, being the SISO case the worst, and constant, independently of the angle, as it is shown in Fig. 22, since a single omni-directional antenna is used. 


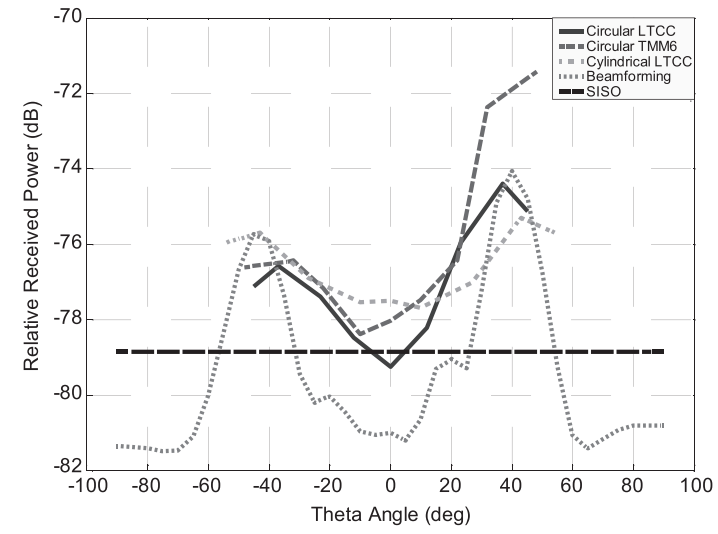

Fig. 22. Relative received power at first position $\left(\theta=0^{\circ}\right)$ in OLOS conditions.

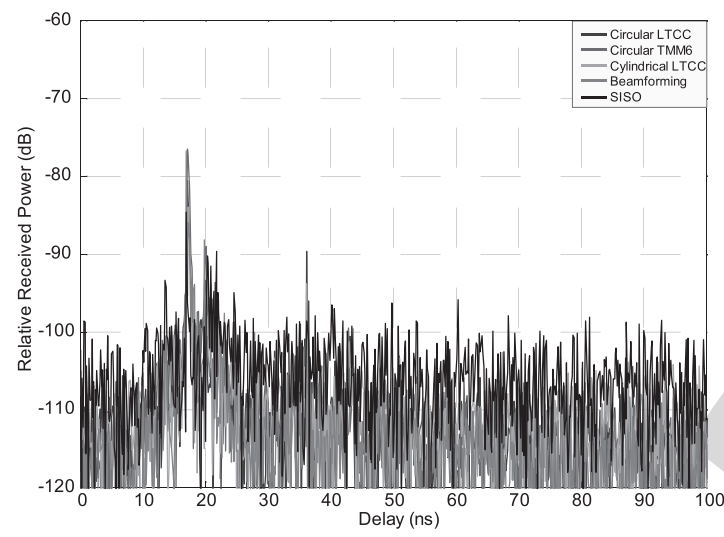

Fig. 23. PDP at first position $\left(\theta=0^{\circ}\right)$ in OLOS conditions.

Regarding the RMS delay spread, the computed values from measurements are very low for all the considered antennas due to the LOS situation, in which the signal is propagating without facing any obstacle. For the lens antennas, the results are very similar, below $1 \mathrm{~ns}$, being for the cylindrical LTCC lens the lowest. However, for the ULA case the RMS delay spread is the double due to the diversity, and for the SISO is even higher because the use of the omni-directional antenna, which has a wider $-3 \mathrm{~dB}$ beamwidth radiation pattern.

For the same setup of the first measured position, we placed an absorbent panel in the middle of the Tx and Rx antennas. Therefore, in this case the direct ray is obstructed by the obstacle. In the same way as in the previous situation, the relative received power in function of the angle and the PDP is plotted for each transmitting antenna in Figs. 22 and 23, respectively. As it is shown in Fig. 23, the direct ray is canceled and a component with lower power than the previous one is received at $17.1 \mathrm{~ns}$. In Fig. 22, it is observed that at $0^{\circ}$, the received power is really low because this path is being obstructed for the absorbent panel. However, thanks to multipath propagation, around $40^{\circ}$ we are receiving a certain amount of power. For this angle, the TMM6 lens is still performing better than the rest of transmitting antennas, despite the ULA is steering the beam to the direction of maximum propagation, but it is clearly receiving less power.
TABLE V

Relative Received Power and RMS Delay Spread Values FOR FIRST POSITION IN OLOS CONDITIONS

\begin{tabular}{|c|c|c|}
\cline { 2 - 3 } \multicolumn{1}{c|}{} & Relative Received Power & RMS Delay Spread \\
\hline Circular LTCC & $-74.38 \mathrm{~dB}$ & $18.81 \mathrm{~ns}$ \\
\hline Circular TMM6 & $-71.42 \mathrm{~dB}$ & $5.77 \mathrm{~ns}$ \\
\hline Cylindrical LTCC & $-75.29 \mathrm{~dB}$ & $36.01 \mathrm{~ns}$ \\
\hline 10-elem. ULA & $-74.05 \mathrm{~dB}$ & $42.78 \mathrm{~ns}$ \\
\hline SISO & $-78.83 \mathrm{~dB}$ & $33.18 \mathrm{~ns}$ \\
\hline
\end{tabular}

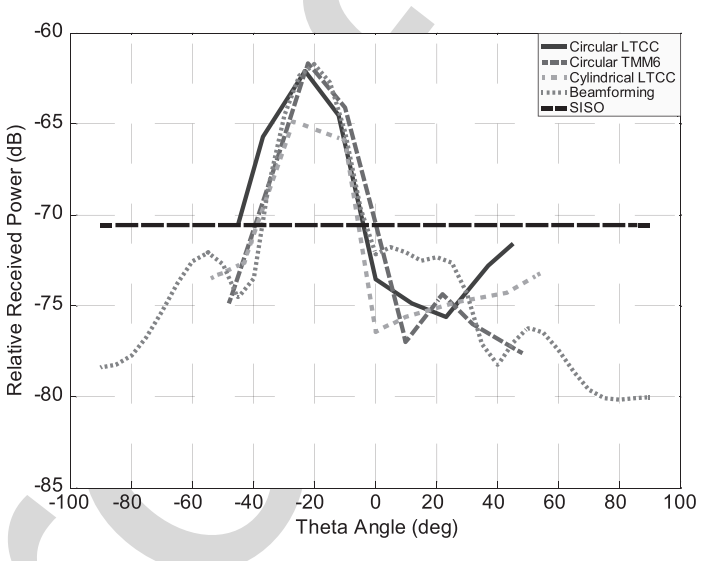

Fig. 24. Relative received power at second position $\left(\theta=22.5^{\circ}\right)$ in LOS conditions.

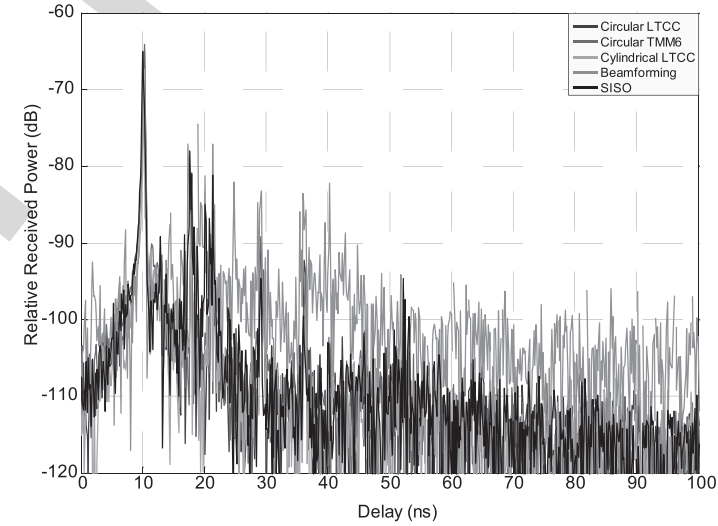

Fig. 25. PDP at second position $\left(\theta=22.5^{\circ}\right)$ in LOS conditions.

Table V shows a summary of the computed values for the relative received power and RMS delay spread for this OLOS situation in the first measurement position. Due to the obstacle, the received power decreases, while the RMS delay spread increases, as it was expected. It is observed that the lowest delay spread is also achieved with the TMM6 lens.

2) Second Position Measurements: As it is depicted in Fig. 19, in the second position situation the $\mathrm{Tx}$ and $\mathrm{Rx}$ antennas are separated $3 \mathrm{~m}$ forming an angle of $22.5^{\circ}$ between them in a LOS condition. In the same way as it has been previously described, the measurements are carried out. Therefore, the relative received power in function of the angle and the PDP are plotted for each different transmitting antenna in Figs. 24 and 25, respectively, and in the Table VI, the computed relative received power and the RMS delay spread are also summarized. 
TABLE VI

Relative Received Power and RMS Delay SPREAd VALUES FOR SECOND POSITION IN LOS CONDITIONS

\begin{tabular}{|c|c|c|}
\cline { 2 - 3 } \multicolumn{1}{c|}{} & Relative Received Power & RMS Delay Spread \\
\hline Circular LTCC & $-62.11 \mathrm{~dB}$ & $1.84 \mathrm{~ns}$ \\
\hline Circular TMM6 & $-61.67 \mathrm{~dB}$ & $1.86 \mathrm{~ns}$ \\
\hline Cylindrical LTCC & $-64.87 \mathrm{~dB}$ & $1.38 \mathrm{~ns}$ \\
\hline 10-elem. ULA & $-61.75 \mathrm{~dB}$ & $12.37 \mathrm{~ns}$ \\
\hline SISO & $-70.54 \mathrm{~dB}$ & $4.11 \mathrm{~ns}$ \\
\hline
\end{tabular}

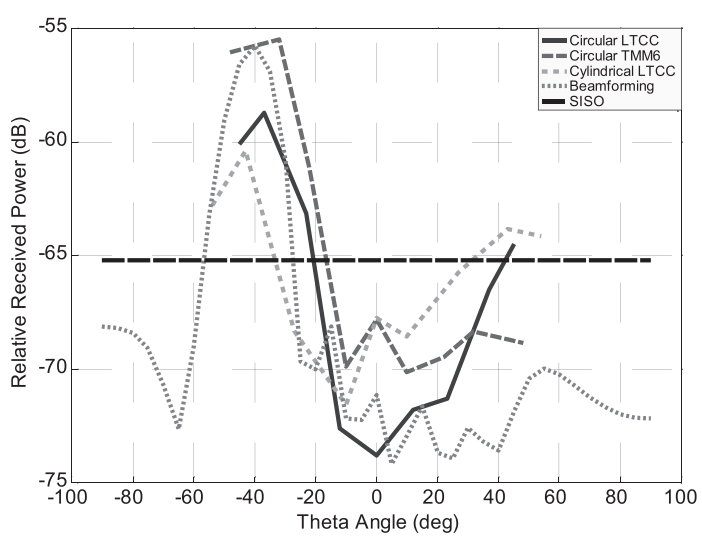

Fig. 26. Relative received power at third position $\left(\theta=45^{\circ}\right)$ in LOS conditions.

As it is shown in Fig. 25, the strongest component is received at $10.1 \mathrm{~ns}$, a similar time delay as for the first position in LOS situation, but the received power is slightly lower because the antennas are forming $22.5^{\circ}$ between them. In this case, the highest received power value is obtained using the TMM6 lens, and the lowest RMS delay spread is achieved with the cylindrical LTCC lens. The power received with the ten-element ULA is almost the same as with the TMM6 lens, however, the RMS delay spread is considerably higher, nearly seven times higher in comparison to TMM6 lens, and up to nine times compared to the value obtained using the cylindrical LTCC lens, which is a remarkable result because directly affects the coherence bandwidth, which in turn limits the capacity in a wireless transmission system.

3) Third Position Measurements: The third position considered in this experimental study is also depicted in Fig. 19, defining a distance of $2.3 \mathrm{~m}$ separating the Tx and Rx antennas and forming an angle of $45^{\circ}$ between them in LOS conditions. For this particular wide-angle case, the measured relative power and the computed PDP are plotted in Figs. 26 and 27, respectively. In addition, the maximum relative received power and the RMS delay spread values are summarized in Table VII for each evaluated Tx antenna. As it is observed, the direct ray with the strongest component (LOS condition) is received at $7.7 \mathrm{~ns}$ for all the considered antennas. The rest of the components arrive delayed due to the multipath propagation, all of them with different levels of attenuation depending on which antenna is used. The maximum received power is centered around $40^{\circ}$, as it is shown in Fig. 26. Once again, the highest received power is achieved with the circular TMM6 lens, despite the wide steering angle in which the

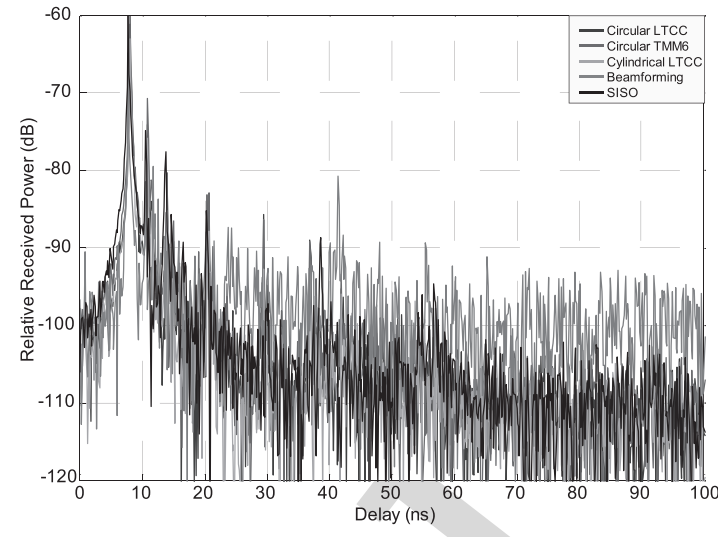

Fig. 27. PDP at third position $\left(\theta=45^{\circ}\right)$ in LOS conditions.

TABLE VII

RELATIVE RECEIVED POWER AND RMS DELAY SPREAD VALUES FOR THIRD POSITION IN LOS CONDITIONS

\begin{tabular}{|c|c|c|}
\cline { 2 - 3 } \multicolumn{1}{c|}{} & Relative Received Power & RMS Delay Spread \\
\hline Circular LTCC & $-58.75 \mathrm{~dB}$ & $1.80 \mathrm{~ns}$ \\
\hline Circular TMM6 & $-55.51 \mathrm{~dB}$ & $1.27 \mathrm{~ns}$ \\
\hline Cylindrical LTCC & $-60.37 \mathrm{~dB}$ & $1.20 \mathrm{~ns}$ \\
\hline 10-elem. ULA & $-55.80 \mathrm{~dB}$ & $18.16 \mathrm{~ns}$ \\
\hline SISO & $-65.21 \mathrm{~dB}$ & $2.92 \mathrm{~ns}$ \\
\hline
\end{tabular}

Rx antenna is placed with respect to the Tx. Regarding the RMS delay spread, the results confirm the previously obtained in other situations, being the cylindrical LTCC lens the best option in order to obtain the lowest value, with a RMS delay spread of $1.2 \mathrm{~ns}, 15$ times lower than the obtained with the ten-element ULA.

\section{Switched-Beam Antenna Based on LTCC DieleCtRic Flat LENSES AND FREQUENCY-SCANNED ARRAYS}

In the last section of this paper, the design of an innovative switched-beam antenna array concept for 5G millimeter-wave applications, based on a practical application of the cylindrically distributed parameters LTCC flat lens, is presented and completely evaluated.

\section{A. Introduction}

As it has been demonstrated, taking advantage of the cylindrical effective parameter distribution of the lens, the beam scanning can be performed in one plane by moving (or selecting) the position of a radiating single element along the gradient-index axis, whereas the beam can be maintained invariant in the other direction, in which the effective parameters are kept constant, despite changing the radiating element position in this particular axis. Therefore, in this way, the beam scanning can be achieved in the constant-index axis of the lens by means of a different technique, a FSSA [21], [22], which it is also introduced in this final paper section, in order to realize not only a 1-D beam scanning but a 2-D beam scanning of high-gain radiation beams, in a compact millimeter-wave 


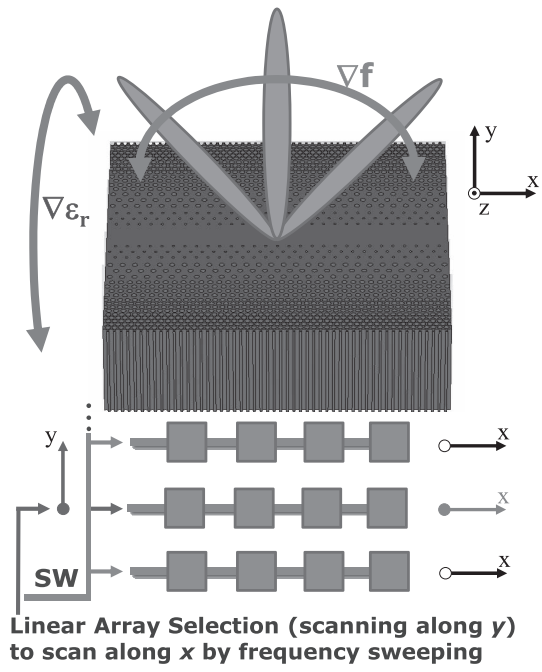

Fig. 28. Switched-beam antenna array concept with cylindrically distributed parameters flat lens and frequency-scanned array to perform beam-scanning in theta and phi, by frequency sweeping or selecting a specific linear array.

antenna solution, easy to integrate in a single monolithic structure with LTCC technology.

Therefore, the theoretical concept for the described behavior of the flat lens in front of a linear array of antennas distributed along the constant-index axis, which is able to scan its beam in this dimension by sweeping the frequency, is shown in Fig. 28.

\section{B. Frequency-Scanned Slot Antenna Array}

Considering that a broadside invariant radiation pattern is obtained in the constant-index axis of the flat lens, despite the feeding aperture is being moved along this axis, a linear frequency-scanned stripline-fed transverse slot antenna array with a particular structure has been designed to achieve beam scanning in one single plane by sweeping the frequency, taking advantage of the huge amount of available bandwidth for communication applications around $60 \mathrm{GHz}$.

1) Frequency-Scanned Slot Array Design and Geometry: In this kind of arrays, the beam-steering capability is obtained controlling the relative phase shift between the array elements by sweeping the operating frequency [21], instead of introducing phase delays by means of bulky and complex phase shifters, as it is common in traditional phased arrays.

The proposed linear array geometry is shown in Fig. 29. It consists of a set of ten transverse slots fed by a meandering stripline, which provides the required phase delay between slot elements in order to steer the beam when the frequency is conveniently changed. The signal is propagating through the stripline and it is coupling energy to each one of the slots, which in turn, is radiating the coupled energy to the free space. In this way, the slots which are closer to the stripline feeding point need to be less coupled than the slots which are far away from this point, because the signal is stronger at the beginning and tends to be smoothly weakened because it is being radiated at every consecutive slot it finds during its propagation. The stripline is terminated with a matched load in order to absorb the remaining power which is not being radiated after the last of the slots, thus avoiding undesired reflections. This array is

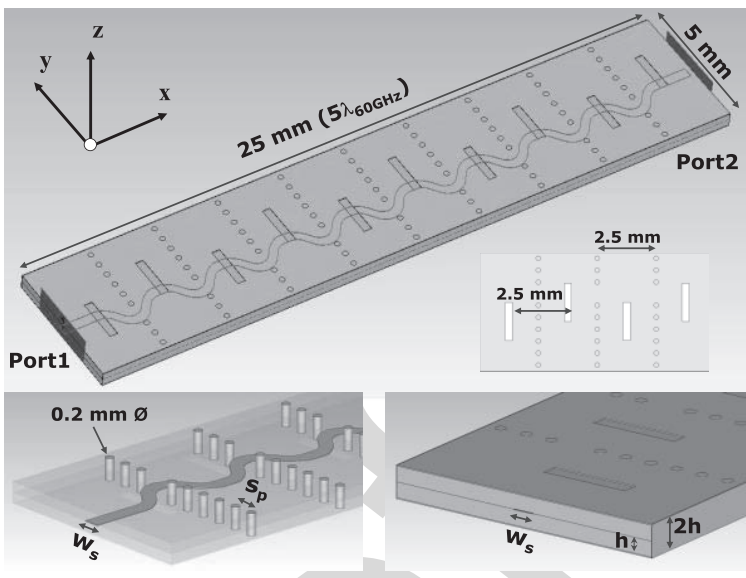

Fig. 29. Frequency-scanned stripline-fed transverse slot antenna array geometry: whole structure (top), and detailed images of the meandering stripline and the pin curtains (left) and two layer structure geometry dimensions (right).

a nonresonant structure, in which traveling waves are used for the excitation of the slots, opposed to resonant or standing wave arrays, in which a short circuit is placed at the end, instead of a matched load.

The total dimensions of this novel stripline-fed slot antenna array are $25 \mathrm{~mm} \times 5 \mathrm{~mm}\left(5 \lambda_{60 \mathrm{GHz}} \times 1 \lambda_{60} \mathrm{GHz}\right)$, with $508 \mu \mathrm{m}$ thickness. It is designed from two different Rogers Duroid $5880\left(\varepsilon_{r}=2.2 ; \tan (\delta)=0.004\right.$ at $60 \mathrm{GHz}$ [23] $)$ substrate layers of $254 \mu \mathrm{m}$ thickness. This substrate was chosen for its low losses and low permittivity values, which facilitate the radiation and improve the overall antenna efficiency.

The slot geometry plane is printed on the top substrate layer, while the meandering stripline and the ground-plane are printed on the bottom layer; thus the feeding line is placed in between top and bottom planes. The slot dimensions are all the same $(1.6 \mathrm{~mm} \times 0.3 \mathrm{~mm})$. The meandering stripline is designed in $370 \mu \mathrm{m}$ width, in order to ensure $50 \Omega$ at the feeding port.

As it is shown in Fig. 29, the ten slots are placed transversal to the feeding stripline, leaving a physical distance of $\lambda_{060 \mathrm{GHz}} / 2$ between them. The meandering stripline length is around $\lambda_{\mathrm{g} 60 \mathrm{GHz}}$ (a wavelength inside the substrate) and guarantees the needed phase delay to perform the desired beam steering with the frequency sweeping from 57 to $66 \mathrm{GHz}$.

Initially, all the ten slots are placed $-0.4 /+0.4 \mathrm{~mm}$ (odd/even slots, respectively) with respect to the array center along the $y$-direction (i.e., the slot feeding position, see Fig. 29), thus providing the same coupling level to all of them. After an iterative optimization process, by using the CST's trust region algorithm, defining a tradeoff between maximum achievable gain and a fixed value of SLLs below $-10 \mathrm{~dB}$, considering the whole frequency band from 57 to $66 \mathrm{GHz}$, the final position along $y$-direction for each individual slot is determined. A transversal pin curtains (see Fig. 29) are placed between slot elements in order to isolate each one from each other to avoid the coupling and suppressing the surface wave propagation between the parallel plates of the array. 


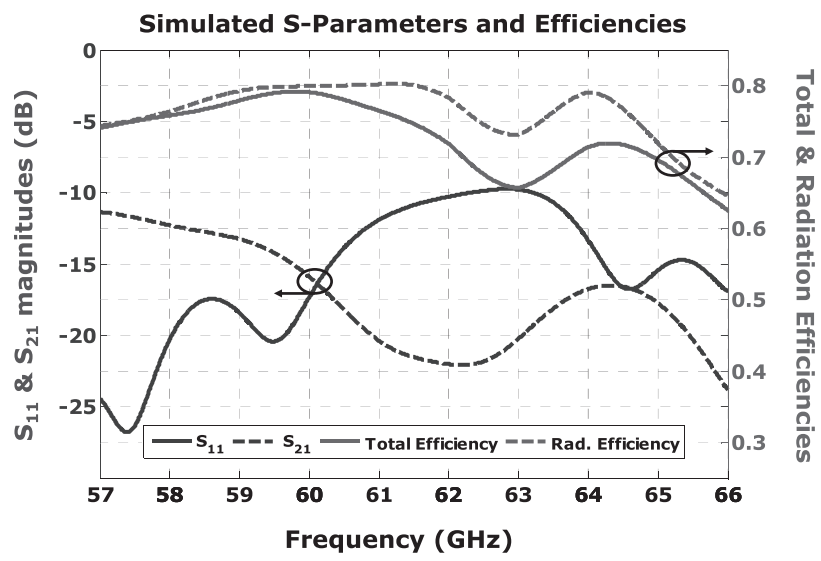

Fig. 30. S-Parameters ( $S_{11}$ and $\left.S_{21}\right)$ and efficiencies (total and radiation) simulation results for the frequency-scanned slot array in the wholeband of interest.

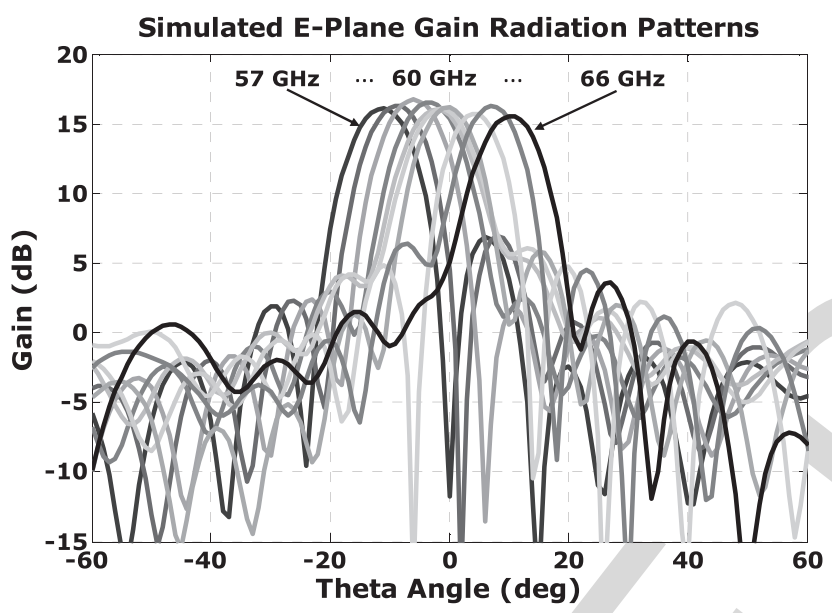

Fig. 31. Simulated E-plane gain radiation patterns obtained sweeping the frequency of the linear slot array, in steps of $1 \mathrm{GHz}$, from 57 to $66 \mathrm{GHz}$.

2) Frequency-Scanned Slot Array Simulation Results: Therefore, the final frequency-scanned slot array design has been simulated using CST Microwave Studio with the timedomain solver from 57 to $66 \mathrm{GHz}$. In Fig. 30, the simulation results of the S-parameters, radiation, and total efficiencies for the frequency-scanned array are plotted. As it is shown, the structure is well-matched since the reflection coefficient $\left(S_{11}\right)$ is below $-10 \mathrm{~dB}$ over the whole frequency band.

The simulated transmission coefficient $\left(S_{21}\right)$ is also below $-10 \mathrm{~dB}$, which means that most part of the input power is being transferred to the antenna from the feeding stripline, and then, radiated to the free-space; likewise, it is supposed that the power is not being trapped into the array structure. Moreover, in this sense, the simulated total and radiation efficiencies are showing values around $70 \%-80 \%$. Note that $S_{22}$ and $S_{12}$ parameters are not plotted due to the symmetry and reciprocity of the design.

The E-plane gain radiation pattern at each frequency, in steps of $1 \mathrm{GHz}$, is plotted in Fig. 31. As it is shown, with the proposed design we are able to scan the maximum of the beam from $-12^{\circ}$ to $+12^{\circ}$, with almost constant gain values

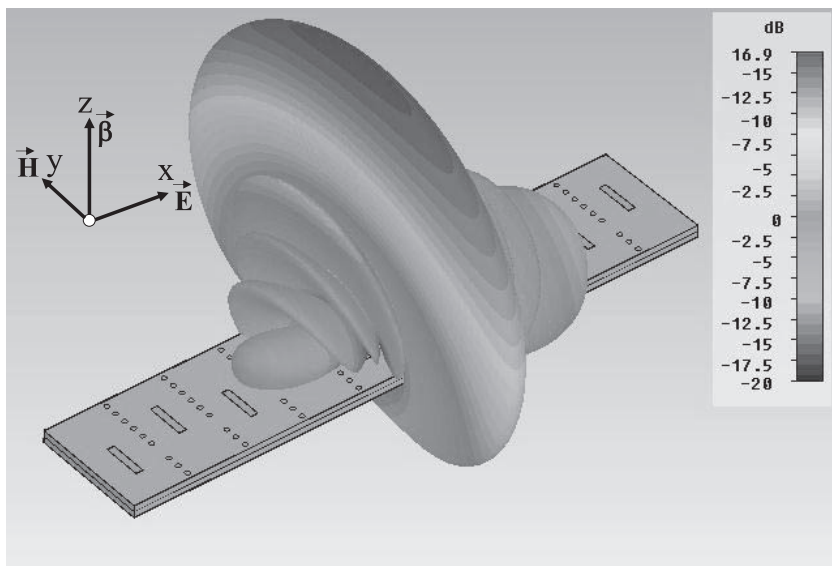

Fig. 32. 3-D representation of the fan-beam obtained in simulation with the frequency-scanned stripline-fed transverse slot antenna array at $60 \mathrm{GHz}$.

around $16 \mathrm{~dB}$, and up to $16.7 \mathrm{~dB}$ gain. From $-15^{\circ}$ to $+14^{\circ}$, we are able to obtain beam scanning with more than $15 \mathrm{~dB}$ gain, and from $-20^{\circ}$ to $+18^{\circ}$, we still have $10 \mathrm{~dB}$. SLL is below $-10 \mathrm{~dB}$ for all the radiation beams and below $-14 \mathrm{~dB}$ in most of the cases, with $-3 \mathrm{~dB}$ beamwidths around $12^{\circ}$.

Because of the linear distribution of the slots along $x$-direction, the frequency-scanned array is also generating a fan-beam radiation pattern having a narrow beamwidth in this specific dimension, while the typical broader beamwidth of a single slot antenna is obtained along the orthogonal $y$-axis, as it is expected. A 3-D representation of the fan-beam radiation pattern obtained with the numerical results of the performed simulations is plotted in Fig. 32. Note that since the linear array is modeled with a set of slot antennas individually linearly polarized in $x$-direction, the whole array structure is performing a linearly $x$-direction polarized radiation pattern as well.

The overall performance of the proposed slot array in simulation is comparable to the obtained with similar designs [22], having even better gain values while using a smaller fractional bandwidth to perform the frequency sweep.

Moreover, its singular novel stripline-fed transverse structure, with the feeding line isolated from outer parts, allows for a better control of the radiated fields in order to optimally illuminate the cylindrically distributed parameters flat lens, also facilitating an easier adaptation of the design if there is a change in the boundaries, or a redesign for higher frequencies is needed.

\section{Complete Switched-Beam Antenna Array}

This section is devoted to numerically evaluate the performance of the complete SWBA array structure based on both presented flat lens and frequency-scanned array.

1) Concept Description and Final Geometry: As it has been demonstrated before, with the help of the cylindrically distributed parameters flat lens it is possible to correct the phase in one single plane in order to focus the radiation beam. Since the FSSA provides a fan-beam radiation pattern, which is easy to steer along its linear structure by sweeping the 


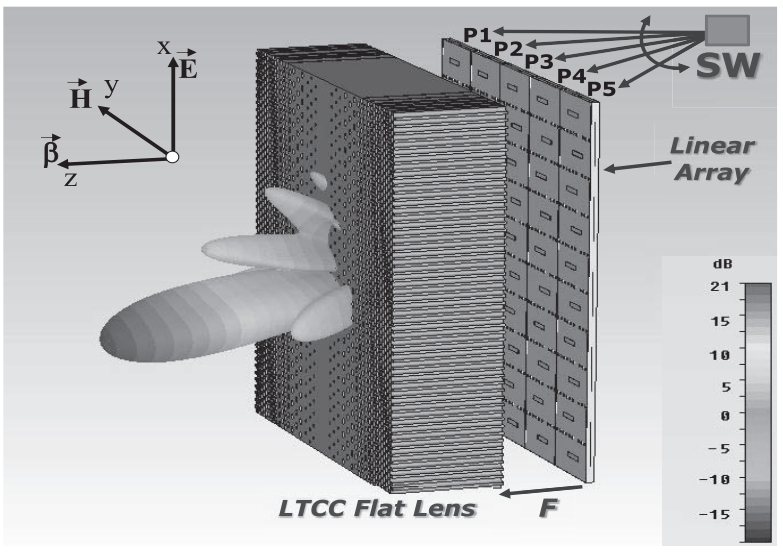

Fig. 33. Complete SWBA array structure for future high data rate $5 \mathrm{G}$ wireless communication applications and 3-D representation of the high-gain pencil beam obtained in numerical simulations.

frequency, if we correctly place this linear array orthogonally to the gradient-index axis of the lens, the final result will lead to a 2-D focused radiation beam, which in turn will allow the beam-scanning easily in 2-D. Therefore, the linear frequencyscanned array model has been replied five times along the gradient-index of the flat lens, placed orthogonally at its focal distance, as it is depicted in Fig. 33.

Since the overall dimensions of a single linear array are $5 \mathrm{~mm} \times 25 \mathrm{~mm}$, and because it is replied five times along $y$-axis (see Fig. 33), the final array planar dimensions are $25 \mathrm{~mm} \times 25 \mathrm{~mm}$, exactly the same square dimensions of the flat lens. The final structure is modeled with five input ports (P1-P5, Fig. 33), and five matched ports $(50 \Omega)$ at the end of each linear array. In this way, the number of switching elements needed if we want to individually select one single port among the five available is significantly reduced, thus in turn decreasing considerably the losses introduced and the complexity of the integration of this kind of electronic components at millimeter-wave frequencies.

2) Complete SWBA Simulation Results: The complete switched-beam antenna array structure has been numerically simulated with CST Microwave Studio in the $60 \mathrm{GHz}$ band, from 57 to $66 \mathrm{GHz}$, to evaluate the final performance of the proposed novel antenna solution. The corresponding E-plane gain radiation patterns obtained by sweeping the frequency from 57 to $66 \mathrm{GHz}$, in steps of $1 \mathrm{GHz}$, are plotted in Fig. 34, for the case of selecting the third port (i.e., the central linear array among the five).

As it is shown, with the proposed solution we are able to increase the maximum achievable gain up to $21.5 \mathrm{~dB}$, with constant gain level over $20 \mathrm{~dB}$, and beam scanning capabilities along the vertical dimension from $-12^{\circ}$ to $+12^{\circ}$ by sweeping the frequency from 57 to $66 \mathrm{GHz}$. SLL are below $-10 \mathrm{~dB}$ for all the beams, with narrow $-3 \mathrm{~dB}$ beamwidths around $11^{\circ}-12^{\circ}$.

The fan-beam radiation pattern generated by the FSSA array is modified by the gradient axis of the lens producing a highgain pencil-beam radiation pattern. A 3-D representation of the pencil-beam radiation pattern obtained with the numerical results of the performed simulations, together with the SWBA

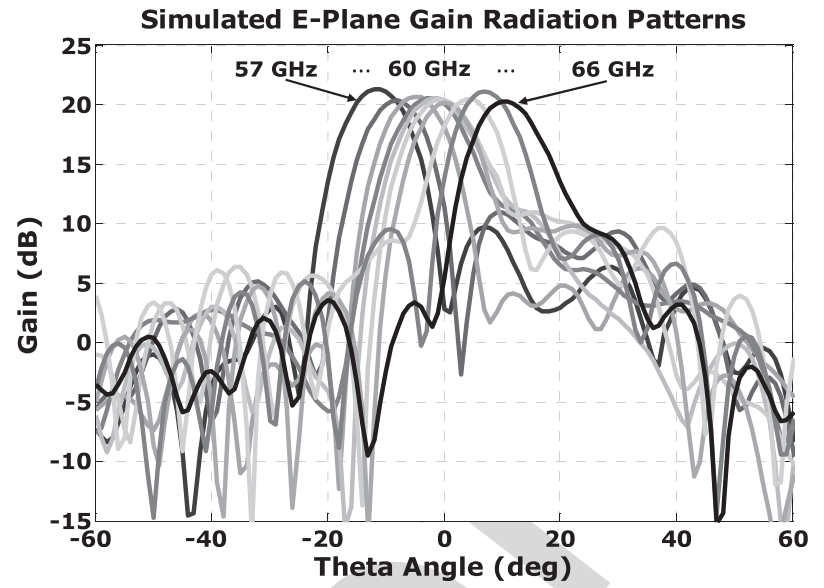

Fig. 34. Simulated E-plane gain radiation patterns obtained sweeping the frequency of P3 of the SWBA array, in steps of $1 \mathrm{GHz}$, from 57 to $66 \mathrm{GHz}$.

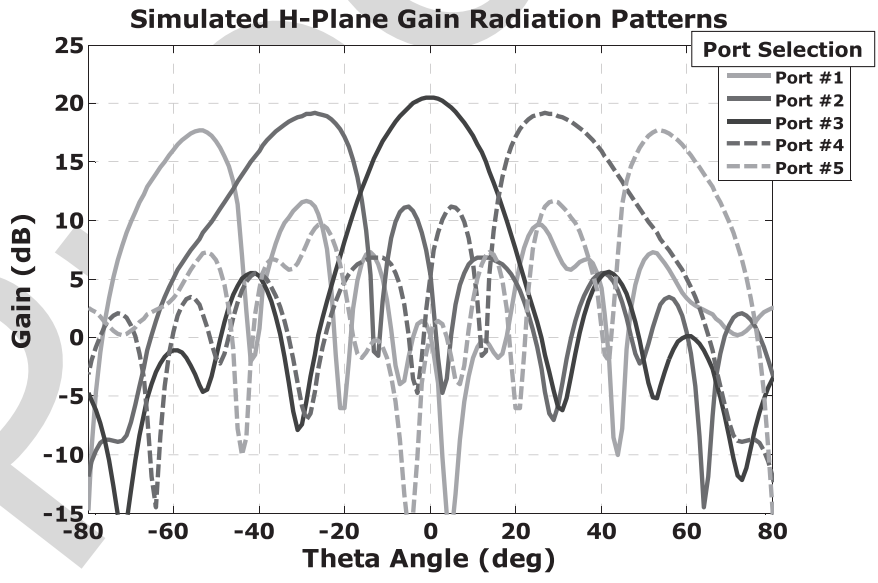

Fig. 35. Simulated H-plane gain radiation patterns at $61 \mathrm{GHz}$ obtained selecting individually each one of the five ports of the SWBA array.

array structure, is plotted in Fig. 33. Theoretically, an infinite number of high-gain pencil beams can be obtained to scan in the vertical direction, while in the horizontal dimension we can pick one of the five different sets of beams, depending on which one of the five ports of the array is selected, as it is plotted in Fig. 35, where the radiation patterns in the H-plane are shown at a frequency of $61 \mathrm{GHz}$ (in which the beams are pointing at $0^{\circ}$ in elevation), to finally cover the scanning in both azimuth and elevation.

In this sense, and in order to show the complete scanning capabilities of the SWBA array, a 3-D representation of the simulated gain radiation patterns obtained selecting individually ports \#3, \#2, and \#1, and changing the frequency at each port to 57, 60, and $66 \mathrm{GHz}$ (low, mid, and high band frequencies, respectively) are plotted in Fig. 36. Given the SWBA array symmetric structure, symmetric radiation patterns pointing rightwards in azimuth are obtained selecting ports \#4 and \#5 instead of ports \#1 and \#2, and therefore are not shown. Alternatively, the complete set of radiation patterns obtained selecting individually each one of the five ports, and sweeping the frequency from 57 to $66 \mathrm{GHz}$, in steps of $1 \mathrm{GHz}$ (ten patterns) at each port, is jointly plotted in Fig. 37. 

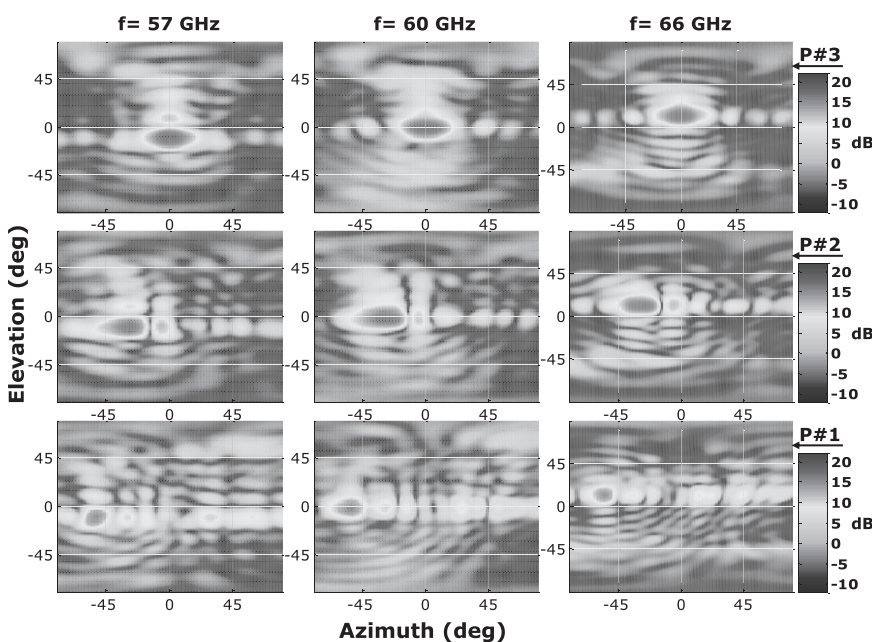

Fig. 36. 3-D representation of the simulated gain patterns obtained with the SWBA selecting individually ports \#3 (first row of the plot), \#2 (second row), and \#1 (third row) at single frequencies of 57, 60, and $66 \mathrm{GHz}$ (columns 1-3).

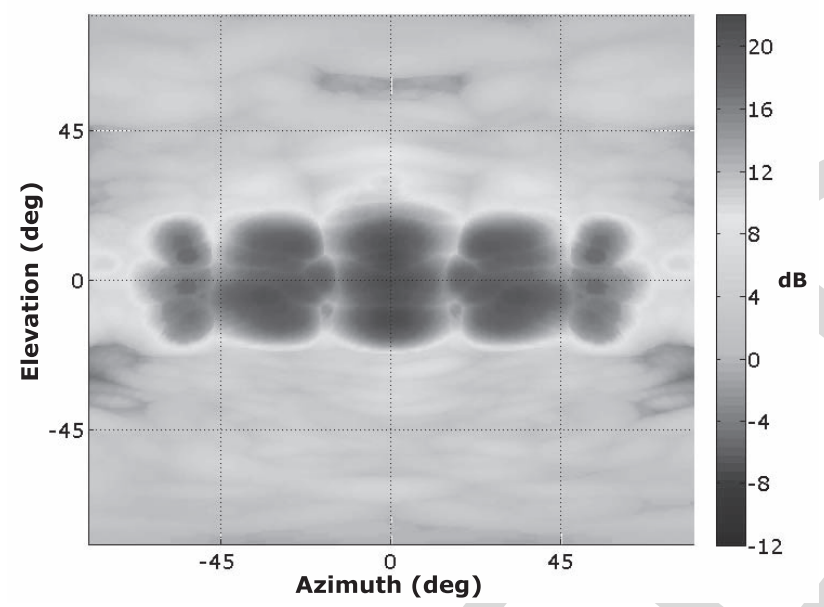

Fig. 37. 3-D joint representation of the complete set of simulated gain radiation patterns obtained with the SWBA selecting each one of the five ports (to scan over azimuth), and sweeping the frequency from 57 to $66 \mathrm{GHz}$ in steps of $1 \mathrm{GHz}$ at each port (to scan over elevation).

As it is observed in Figs. 36 and 37, our numerical results indicate that we are able to scan a high-gain radiation pencil beam (up to 21-21.5 $\mathrm{dB}$ in the broadside direction) from around $-55^{\circ}$ to $+55^{\circ}$ in azimuth, by selecting one single port of the five available, and from around $-20^{\circ}$ to $+20^{\circ}$ in elevation, by sweeping the frequency from 57 to $66 \mathrm{GHz}$ (the maximum of the beams in elevation is going from $-12^{\circ}$ to $+12^{\circ}$, as it is clearly shown in Fig. 34 , but at $\pm 20^{\circ}$ we still achieve up to $15 \mathrm{~dB}$ gain. The simulation results also indicate that the whole structure is well matched $\left(S_{11}<10 \mathrm{~dB}\right)$ for the entire frequency band, as it was expected, obtaining the same simulation results as the previously reported or the single FSSA array alone (thus are not plotted), because the lens, which is placed at $6.25 \mathrm{~mm}$ (focal distance) from the slot array, is not altering or modifying the FSSA array behavior in this sense. Likewise, simulated total and radiation efficiencies results are also quite similar to the previously reported for the

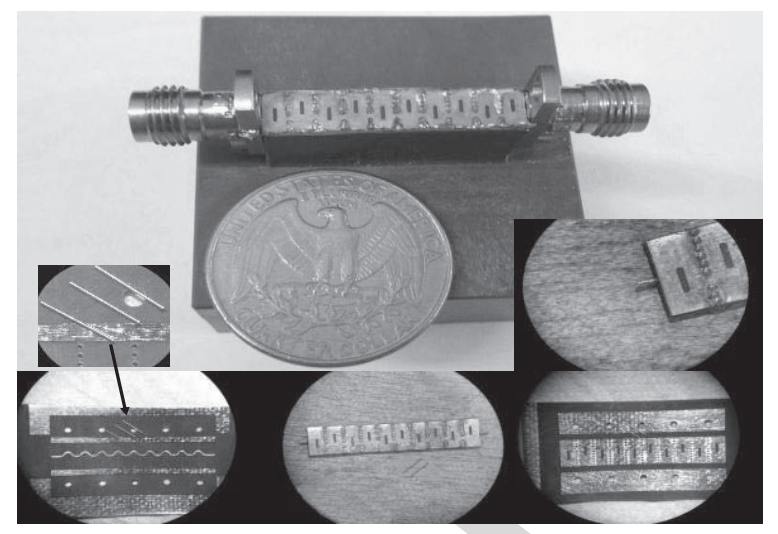

Fig. 38. FSSA connectorized and mounted over a PVC support. Some microscopic images of the bottom layer before being stacked (with the meandering stripline), complete design before connectorization, top layer (with the slots), and detailed image of the signal pin together with the first two slots and the first pin curtain, are shown in the insets.

FSSA array evaluated individually, since a low-loss substrate is used to model the lens, and therefore are not shown either.

\section{FSSA and SWBA Array Prototypes Fabrication}

1) Traveling-Wave Frequency-Scanned Slot Antenna Array: A prototype of the FSSA array has been fabricated at UPC facilities using standard photo-etching techniques on two Rogers Duroid 5880 substrate layers of $254 \mu \mathrm{m}$ thickness. All FSSA array dimensions are specified in previous sections of this paper. A photograph of the fabricated prototype, mounted over a PVC support to facilitate its electromagnetic characterization with our measurement setup, is shown in Fig. 38.

A low insertion loss $1.85 \mathrm{~mm}$ flange jack connector is mated to each signal pin of the FSSA array. The transversal pin curtains are made from $0.2 \mathrm{~mm}$ diameter brass rivets, which are separated $0.5 \mathrm{~mm}$ center to center; they are arranged in line, as it is depicted in Fig. 29, in two different groups of six and three pins, leaving a central space between them to allow the meandering stripline pass through. The pins are soldered interconnecting the top plane, in which the slots are printed, to the bottom ground plane, going through the two substrates.

2) Complete Switched Beam Antenna Array: Finally, the FSSA structure for the complete SBWA array, in which the single array is five times replied along its short dimension, has also been fabricated. A photograph of the prototype also mounted on a PVC support is shown in Fig. 39.

Therefore, the complete SWBA array structure, and the different parts of the final design (e.g., the five input ports connectorized, with their corresponding matched resistors (r1-r5) soldered at the end of each meandering line) are identified, together with the cylindrically distributed permittivity lens placed over the array at its focal distance $F$ with the help of a Rohacell foam structure is also shown in Fig. 39.

\section{E. Complete SWBA Measurement Results}

A complete set of measurements have been carried out at UPC facilities in order to assess the performance of the proposed antenna solution for millimeter-wave applications. 


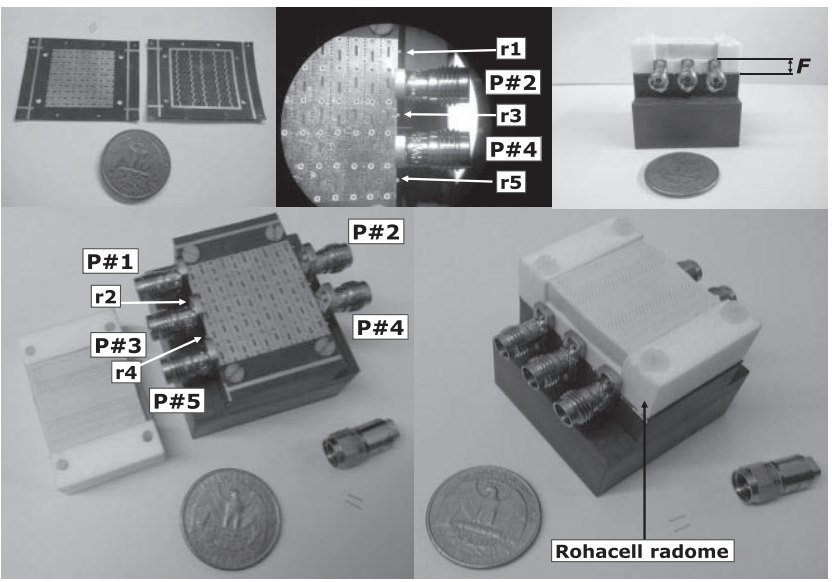

Fig. 39. Complete five-input port SWBA array for $60 \mathrm{GHz}$ WPAN applications, able to perform 2-D scanning of high-gain beams, mounted on a PVC support. Images of the five-input port FSSA fabrication process are shown in the insets.

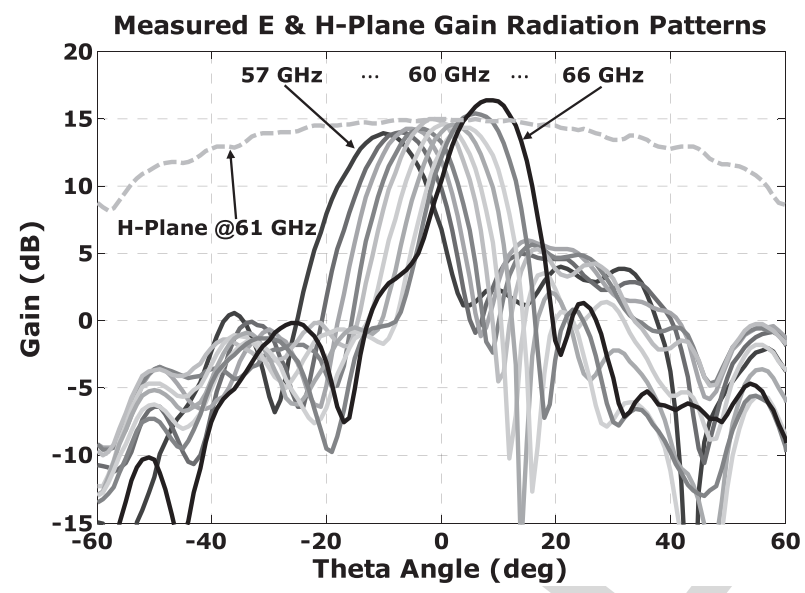

Fig. 40. Measured E-plane (solid lines) and H-plane (dashed lines) radiation patterns of the FSSA sweeping the frequency, from 57 to $66 \mathrm{GHz}$, in steps of $1 \mathrm{GHz}$
First, the FSSA array is characterized separately and, finally, the complete SWBA array structure is completely tested in the whole $60 \mathrm{GHz}$ WPAN frequency band.

1) FSSA array Performance Evaluation: In the same way as it has been previously realized, the radiation pattern measurements for the FSSA array have been carried out at AntennaLab facilities of the UPC with the same far-field setup depicted in Fig. 11. The measured E-plane gain radiation patterns obtained from 57 to $66 \mathrm{GHz}$, in steps of $1 \mathrm{GHz}$, are plotted in Fig. 40. As it is observed, with the fabricated prototype we are able to scan the maximum of the beam from $-10^{\circ}$ to $+9^{\circ}$, with remarkable gain values above $14 \mathrm{~dB}$ for all scanning angles, with a maximum of $16.4 \mathrm{~dB}$ at $66 \mathrm{GHz}$, with $10^{\circ}$ of beam steering. Moreover, we are able to scan the radiation beam from $-18^{\circ}$ to $+16^{\circ}$ with at least $10 \mathrm{~dB}$ gain. SLL are below $-10 \mathrm{~dB}$ in most of the cases and around $-8.5 \mathrm{~dB}$ in the worst case, at $59 \mathrm{GHz}$, with $-3 \mathrm{~dB}$ beamwidths between $11^{\circ}$ and $13^{\circ}$.

The measured H-plane gain radiation pattern, which is the typical broad radiation pattern obtained for a single slot antenna, as it was expected, is also plotted (dashed line)

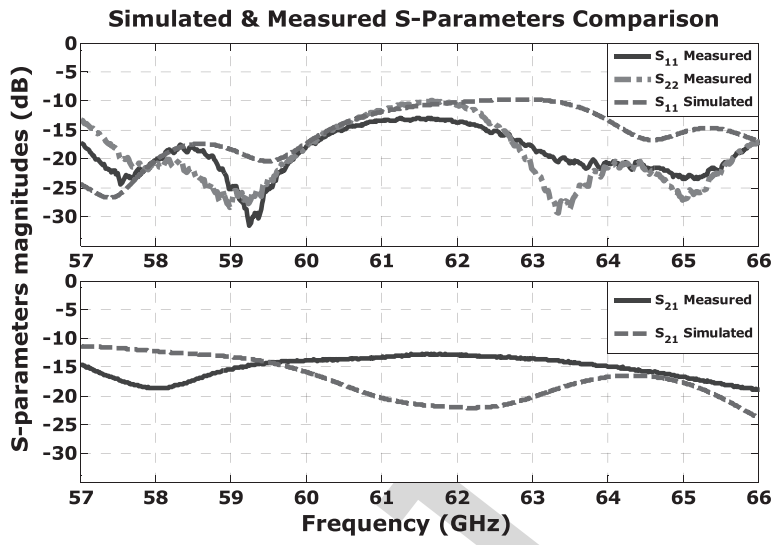

Fig. 41. Simulated and measured S-parameters comparison for the FSSA array in the whole frequency band of interest.

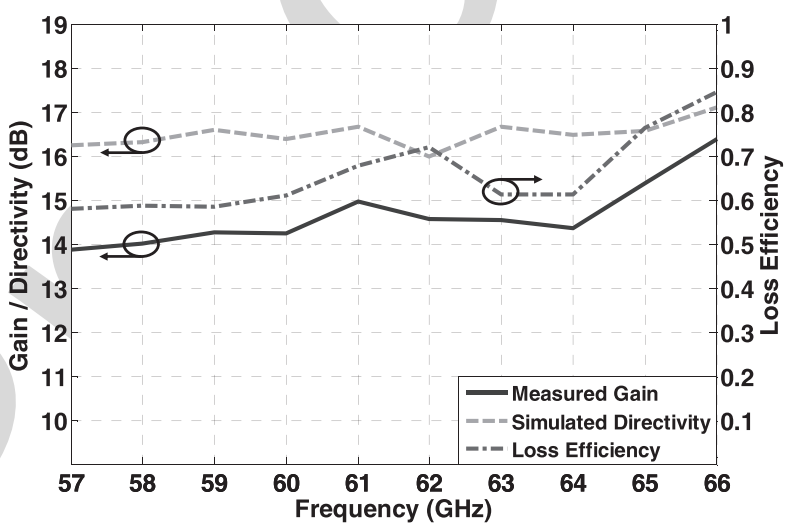

Fig. 42. Computed loss efficiency by using measured gain and simulated directivity results of the FSSA array in the whole frequency band of interest.

in Fig. 40, for a frequency of $61 \mathrm{GHz}$, in which the beam is pointing at $0^{\circ}$ in elevation, thus allowing the measurement in the $x z$ plane with our setup (see Fig. 11). Additionally, the measured cross-polarization level of the FSSA array is around $-20 \mathrm{~dB}$ below copolarization level.

The measured S-parameters of the FSSA array, after applying a full two-port SOLT calibration in the Agilent N5247A VNA, are plotted in Fig. 41 for the whole frequency band of interest. As it is shown, there is a very good agreement between simulation and measurement results; the FSSA is well matched and, since the measured transmission coefficient $\left(S_{21} / S_{12}\right)$ is below $-10 \mathrm{~dB}$, it is supposed that most part of the power is being radiated from the slots to the freespace, as we previously pointed out.

Going further in this sense, the estimated loss efficiency is plotted in Fig. 42, also computed from CST simulation results of the directivity, and measured gain values in the whole WPAN frequency band. The efficiency values, above $60 \%$, and up to $80 \%$, confirm the hypothesis that most part of the power is being correctly radiated. Also note that the measured $S_{11}$ and $S_{22}$ parameters are not identical because of small imperfections in the FSSA array fabrication process.

2) Complete Switched-Beam Antenna Array Characterization: In this section, the electromagnetic characterization of 


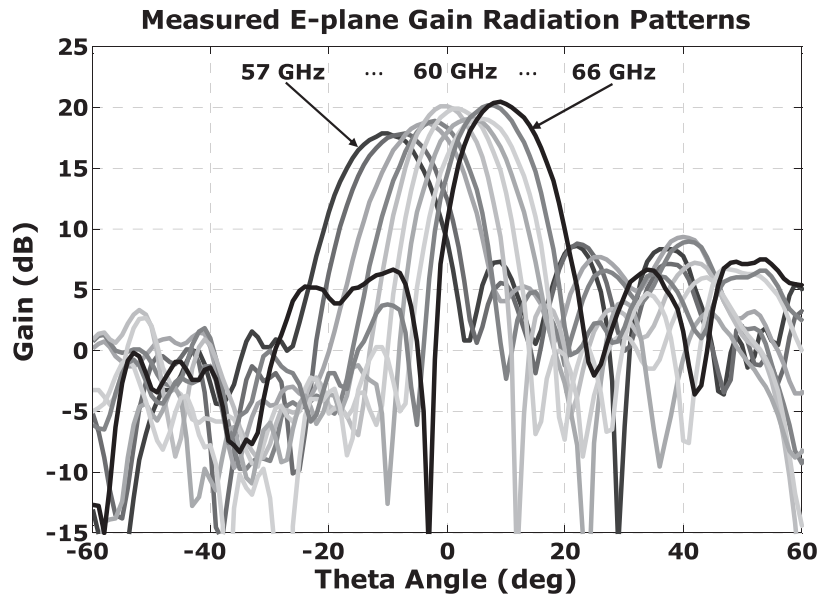

Fig. 43. Measured E-plane gain radiation patterns obtained sweeping the frequency, from 57 to $66 \mathrm{GHz}$, in steps of $1 \mathrm{GHz}$, selecting the third port (central linear array) of the SWBA array.

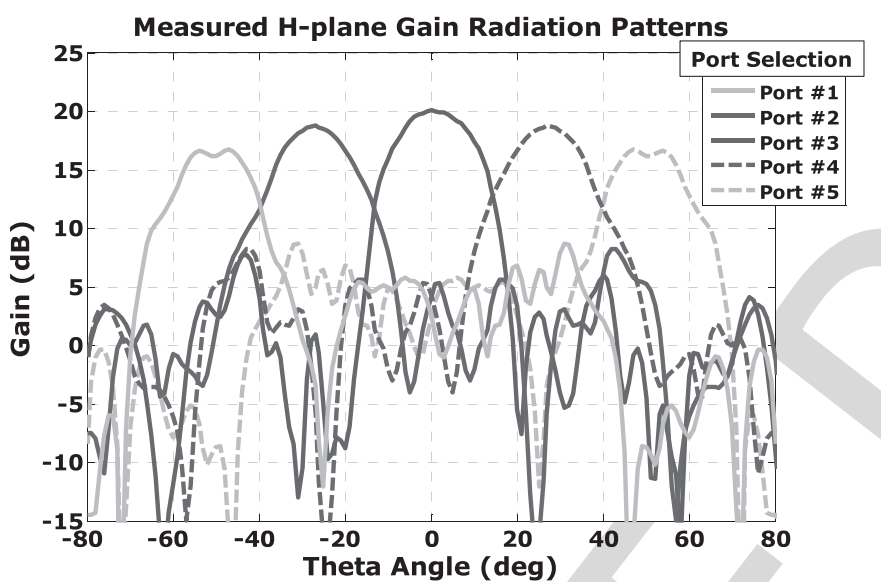

Fig. 44. Measured H-plane gain radiation patterns obtained at a frequency of $61 \mathrm{GHz}$, selecting individually each one of the five ports of the SWBA array.

the complete SWBA array structure, based on the previously presented and evaluated dielectric flat lens and FSSA array in its complete five-input port configuration, has also been carried out at AntennaLab facilities. A photograph of the final SWBA array prototype mounted on a PVC-Rohacell support to facilitate the measurements is shown in Fig. 39.

Similarly, as in previous sections, the gain radiation patterns of the SWBA array have been measured for different antenna configurations, from 57 to $66 \mathrm{GHz}$, in steps of $1 \mathrm{GHz}$, with the setup shown in Fig. 11. The E-plane gain radiation patterns obtained selecting the third port (central linear array of the five available), and sweeping the frequency are plotted in Fig. 43. The H-plane radiation patterns obtained selecting each one of the five ports separately, at a fixed frequency of $61 \mathrm{GHz}$ in which the beams are pointing $0^{\circ}$ in elevation, thus having their maximums in the $x z$ plane, are plotted in Fig. 44.

As it is observed in Fig. 43, by sweeping the frequency, with the fabricated SWBA array prototype we are able to scan the maximum of the beam from $-10^{\circ}$ to $+9^{\circ}$, with highgain values around $18 \mathrm{~dB}$ and above for all the scanning
TABLE VIII

Summary of SWBA ARray Performance at $60 \mathrm{GHz}$ Band SElecting Port \#3 (Central Port)

\begin{tabular}{|c|c|c|c|c|}
\hline Frequency & $\mathbf{G}_{\text {max. }}$ & $\left(\boldsymbol{\theta}^{\circ}\right)_{\text {scan }}$ & $\Delta \theta_{-3 \mathrm{~dB}}$ & SLL \\
\hline $57 \mathrm{GHz}$ & $17.8 \mathrm{~dB}$ & $-10^{\circ}$ & $13^{\circ}$ & $-9.3 \mathrm{~dB}$ \\
\hline $58 \mathrm{GHz}$ & $17.79 \mathrm{~dB}$ & $-7^{\circ}$ & $12.5^{\circ}$ & $-9.25 \mathrm{~dB}$ \\
\hline $59 \mathrm{GHz}$ & $18.7 \mathrm{~dB}$ & $-4^{\circ}$ & $12^{\circ}$ & $-9.4 \mathrm{~dB}$ \\
\hline $60 \mathrm{GHz}$ & $18.81 \mathrm{~dB}$ & $-2^{\circ}$ & $11.5^{\circ}$ & $-9.9 \mathrm{~dB}$ \\
\hline $61 \mathrm{GHz}$ & $20.05 \mathrm{~dB}$ & $0^{\circ}$ & $11.5^{\circ}$ & $-12.95 \mathrm{~dB}$ \\
\hline $62 \mathrm{GHz}$ & $19.87 \mathrm{~dB}$ & $+2^{\circ}$ & $11.5^{\circ}$ & $-14 \mathrm{~dB}$ \\
\hline $63 \mathrm{GHz}$ & $19.03 \mathrm{~dB}$ & $+4^{\circ}$ & $11.5^{\circ}$ & $-15.1 \mathrm{~dB}$ \\
\hline $64 \mathrm{GHz}$ & $19.02 \mathrm{~dB}$ & $+6^{\circ}$ & $11.5^{\circ}$ & $-12.3 \mathrm{~dB}$ \\
\hline $65 \mathrm{GHz}$ & $20.14 \mathrm{~dB}$ & $+7^{\circ}$ & $11.5^{\circ}$ & $-13.9 \mathrm{~dB}$ \\
\hline $66 \mathrm{GHz}$ & $20.4 \mathrm{~dB}$ & $+9^{\circ}$ & $11.5^{\circ}$ & $-13 \mathrm{~dB}$ \\
\hline
\end{tabular}

angles, and up to $20.4 \mathrm{~dB}$ at $66 \mathrm{GHz}$, when the beam is 1111 steered at $+9^{\circ}$. It is also remarkable that for wider scanning angles, from $-21^{\circ}$ to $+20^{\circ}$, we still have at least $10 \mathrm{~dB}$ gain. SLL are, at least, below $-9.25 \mathrm{~dB}$ in the worst case, and below $-12 \mathrm{~dB}$ in general, with $-3 \mathrm{~dB}$ beamwidths between $11.5^{\circ}$ and $13^{\circ}$. To facilitate the reading, the measured radiation pattern parameters of the SWBA array (maximum gain for each beam $\left(G_{\max }\right)$, scanning angles $\left(\theta^{\circ}\right.$ scan $)$, halfpower beamwidths $\left(\Delta \theta_{-3} \mathrm{~dB}\right)$, and SLL) are summarized in Table VIII.

Additionally, the measured cross-polarization level is around $-20 \mathrm{~dB}$ below copolarization level, as in the case of the FSSA array, because the lens is not affecting in this sense the performance of the combination.

As it is also observed from Fig. 44, selecting each one of the five ports, we are able to scan a high-gain radiation beam from $-54^{\circ}$ to $+54^{\circ}$ in the azimuth plane, obtaining more than $16.5 \mathrm{~dB}$ for this wide scanning angle, and still having $10 \mathrm{~dB}$ gain at $\pm 65^{\circ}$. In the broadside direction we achieve a considerable value over $20 \mathrm{~dB}$ gain. Therefore, five different sets of high-gain radiation beams can be selected to scan in the azimuth plane from $-54^{\circ}$ to $+54^{\circ}$, while at the same time an infinite number of beams can be generated in the elevation plane to scan from $-10^{\circ}$ to $+9^{\circ}$ with around $18-20 \mathrm{~dB}$ gain. In general, very good agreement is observed between the obtained radiation pattern measurement results in both planes and the estimated in advance from numerical simulations. Thus, despite we are not able to measure the complete 3-D gain radiation patterns for the SWBA array, the 3-D representation of the complete set of simulated gain radiation patterns plotted in previous Figs. 36 and 37, seems to be an accurate estimation, since the observed agreement between measurements and simulations in the E-plane and $\mathrm{H}$-plane cuts is very good.

The reflection coefficients $\left(S_{11}, S_{22}, S_{33}, S_{44}\right.$, and $\left.S_{55}\right)$ of the SWBA array for the five input ports have also been measured, obtaining approximately the same measurement results as for the $S_{11}$ of the FSSA array plotted in Fig. 41, because the lens placed at focal distance is not affecting the performance in this sense, and therefore are not shown due to space constraints.

Finally, the estimated loss efficiency of the SWBA array is also reported in Fig. 45, computed again from CST simulation results of the directivity and measured gain values, since 


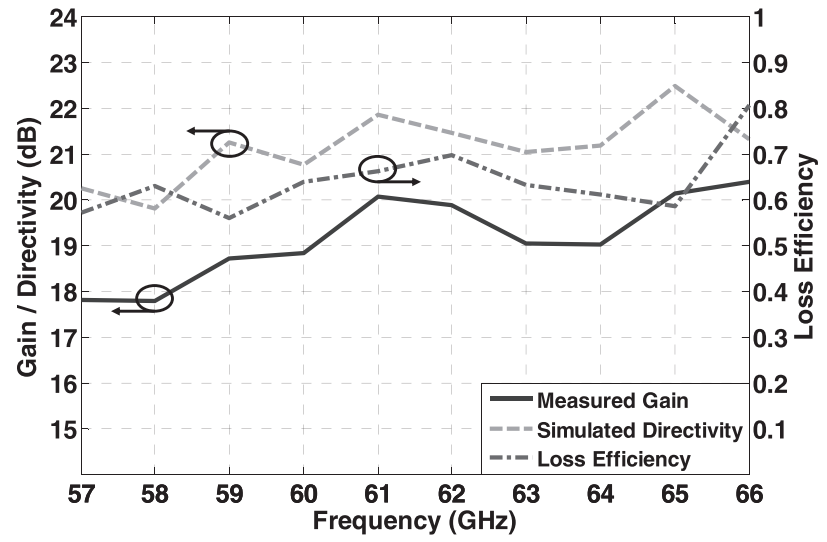

Fig. 45. Computed loss efficiency by using measured gain and simulated directivity results of the SWBA array in the whole frequency band of interest.

with our measurement setup we are not able to measure the complete 3-D radiation patterns in order to integrate the whole power to obtain directly the directivity or the efficiency. As it is observed, good values around $60 \%-70 \%$ and above are estimated in the whole frequency band of interest, also confirming the previously obtained simulation results. Moreover, very good bandwidth performance is also observed in Fig. 45, with gain slightly increasing in frequency, thus also validating the previously reported numerical results.

\section{CONCLUSION}

The design, numerical analysis, LTCC fabrication, and full experimental verification of new inhomogeneous gradientindex dielectric flat lens antennas for future high data rate 5G millimeter-wave wireless communication systems have been presented. Two novel dielectric flat lenses with their effective parameters circularly and cylindrically distributed to provide high-gain pencil-beam and fan-beam radiation patterns, respectively, are designed and fabricated in LTCC technology to allow beam-scanning along both theta and phi directions, despite their planar antenna profile implementation.

The two new LTCC dielectric flat lens antennas have been exhaustively evaluated and compared to a previously introduced TMM6 material flat lens [11], showing in all cases very good performance in terms of radiation pattern parameters: maximum measured gain (between 15 and $18 \mathrm{~dB}$ ), beamsteering capabilities in both planes (between approximately $-50^{\circ}$ and $+50^{\circ}$ ), and low SLL (below $-10 \mathrm{~dB}$ in most of the cases and below -15 and $-17.5 \mathrm{~dB}$ for the broadside direction); estimated efficiencies (over 70\%-80\%), impedance matching, and broadband behavior in the whole frequency band of interest $(57-66 \mathrm{GHz})$.

Additionally, a TDS system has been used to practically evaluate the permittivity profile achieved with the LTCC manufacturing process, which, to our best knowledge, has never been proved before, and even less stacking up to 31 layers of dielectric material, obtaining very good results to confirm the feasibility of fabricating inhomogeneous gradient-index lenses with a desired permittivity profile and planar structure in a mass production technology. The potential integration of the presented dielectric flat lenses in a complete antenna solution with a layer of radiating elements to create a single monolithic structure in LTCC technology has been confirmed as feasible.

Then, the performance of the considered lenses has also been experimentally evaluated and compared to a ten-element ULA of omni-directional antennas applying a beamforming technique, and to a single omni-directional antenna in real $60 \mathrm{GHz}$ WPAN indoor environment under LOS and OLOS conditions, obtaining remarkable results in terms of measured received power and RMS delay spread.

It has been practically demonstrated that in a real millimeter-wave communication scenario the best results in terms of relative received power are achieved in all the considered cases, despite the wide steering angle in which Rx antenna is placed respect to the Tx, with the TMM6 flat lens, closely followed by the circular LTCC lens, and in any case improving the results obtained with the ten-element ULA.

Moreover, the experimental analysis also indicate that in terms of RMS delay spread, the best results are obtained with the cylindrically distributed parameters flat lens, which provides a steerable fan-beam radiation pattern, a remarkable result because enhances the coherence bandwidth to improve the capacity in a wireless transmission system. In this sense, the measured RMS delay spread can be up to 15 times smaller using the proposed cylindrical LTCC flat lens compared to the RMS delay spread obtained with the virtual ULA, when, in a LOS situation, a wide angle between Tx and Rx is established.

Additionally, the complexity in the implementation of the proposed LTCC-based lens antenna solution, which is considerably lower compared to the difficulty in the implementation of beam-forming techniques for phased-array antennas, has also to be taken into account as an important point. It has been experimentally demonstrated their practical application as smart antenna solution for high data rate $5 \mathrm{G}$ millimeterwave commercial systems, not only for mobile devices such as tablets, laptops, or other similar medium-sized devices but also as a possible solution for APs, or even for outdoor BSs, due to their planar antenna configuration and 2-D scanning capability of high-gain radiation beams.

Finally, in order to propose and evaluate a practical application of the introduced lenses for an antenna system, a new switched beam antenna array concept based on the novel LTCC dielectric flat lens with the permittivity cylindrically distributed, and on a traveling-wave FSSA has been introduced, numerically investigated, fabricated, and successfully practically assessed for future $5 \mathrm{G}$ applications at $60 \mathrm{GHz}$ band. The dielectric flat lens and the frequency-scanned array have been exhaustively tested, first separately, and after that together as the complete SWBA array, showing in all cases very good performance in terms of radiation pattern parameters, beamsteering capabilities in both theta and phi planes, measured gain values, efficiencies, impedance matching, and broadband behavior in the whole frequency band of interest $(57-66 \mathrm{GHz})$.

The potential integration of the proposed complete antenna solution in a single monolithic structure has been demonstrated. This technology is suitable and allows mass production for a flat antenna structure such as the proposed in this paper, which is very interesting in order to integrate the solution in compact millimeter-wave wireless mobile devices. 
In contrast to other antenna alternatives, with the proposed solution we are able to scan high-gain radiation beams in both azimuth and elevation planes, necessary for supporting high data rate transmissions ( $>1.5 \mathrm{Gbps})$ as it is recommended in the IEEE 802.15.3c standard, and additionally avoiding the need of high number of integrated RF switches to perform such 2-D radiation pattern reconfiguration.

\section{REFERENCES}

[1] T. S. Rappaport et al., "Millimeter wave mobile communications for 5G cellular: It will work!" IEEE Access, vol. 1, pp. 335-349, May 2013.

[2] J. G. Andrews et al., "What will 5G be?" IEEE J. Sel. Areas Commun., vol. 32, no. 6, pp. 1065-1082, Jun. 2014.

[3] K. Chandra, R. V. Prasad, B. Quang, and I. G. M. M. Niemegeers, "CogCell: Cognitive interplay between $60 \mathrm{GHz}$ picocells and 2.4/5 GHz hotspots in the 5G era," IEEE Commun. Mag., vol. 53, no. 7, pp. 50-56, Jul. 2015.

[4] T. S. Rappaport, G. R. Maccartney, M. K. Samimi, and S. Sun, "Wideband millimeter-wave propagation measurements and channel models for future wireless communication system design," IEEE Trans. Commun., vol. 63, no. 9, pp. 3029-3056, Sep. 2015.

[5] R. Fisher, "60 GHz WPAN standardization within IEEE 802.15.3c," in Proc. Int. Symp. Signals, Syst. Electron. (ISSSE), Jul. 2007, pp. 103-105.

[6] S. Sun, G. R. MacCartney, M. K. Samimi, and T. S. Rappaport, "Synthesizing omnidirectional antenna patterns, received power and path loss from directional antennas for 5G millimeter-wave communications," in Proc. IEEE Global Commun. Conf. (GLOBECOM), San Diego, CA, USA, Dec. 2015, pp. 1-7.

[7] T. S. Rappaport, J. N. Murdock, and F. Gutierrez, Jr., "State of the art in 60-GHz integrated circuits and systems for wireless communications," Proc. IEEE, vol. 99, no. 8, pp. 1390-1436, Aug. 2011.

[8] J. R. Costa, E. B. Lima, and C. A. Fernandes, "Compact beamsteerable lens antenna for $60-\mathrm{GHz}$ wireless communications," IEEE Trans. Antennas Propag., vol. 57, no. 10, pp. 2926-2933, Oct. 2009.

[9] M. Imbert, A. Papio, F. De Flaviis, L. Jofre, and J. Romeu, "Design and performance evaluation of a switched-beam antenna array for $60 \mathrm{GHz}$ WPAN applications," in Proc. 9th Eur. Conf. Antennas Propag. (EuCAP), Apr. 2015, pp. 1-5.

[10] A. Artemenko, A. Maltsev, A. Mozharovskiy, A. Sevastyanov, V. Ssorin, and R. Maslennikov, "Millimeter-wave electronically steerable integrated lens antennas for WLAN/WPAN applications," IEEE Trans. Antennas Propag., vol. 61, no. 4, pp. 1665-1671, Apr. 2013.

[11] M. Imbert, A. Papió, F. D. Flaviis, L. Jofre, and J. Romeu, "Design and performance evaluation of a dielectric flat lens antenna for millimeterwave applications," IEEE Antennas Wireless Propag. Lett., vol. 14, pp. 342-345, 2015.

[12] A. Petosa and A. Ittipiboon, "Design and performance of a perforated dielectric Fresnel lens," IEE Proc.-Microw., Antennas Propag., vol. 150, no. 5, pp. 309-314, Oct. 2003.

[13] A.-E. Mahmoud, W. Hong, Y. Zhang, and A. Kishk, "W-band mutlilayer perforated dielectric substrate lens," IEEE Antennas Wireless Propag. Lett., vol. 13, pp. 734-737, 2014.

[14] M. K. T. Al-Nuaimi and W. Hong, "Discrete dielectric reflectarray and lens for E-band with different feed," IEEE Antennas Wireless Propag. Lett., vol. 13, pp. 947-950, 2014.

[15] P. F. M. Smulders, "Statistical characterization of $60-\mathrm{GHz}$ indoor radio channels," IEEE Trans. Antennas Propag., vol. 57, no. 10, pp. 2820-2829, Oct. 2009.

[16] A. Maltsev, R. Maslennikov, A. Sevastyanov, A. Khoryaev, and A. Lomayev, "Experimental investigations of $60 \mathrm{GHz}$ WLAN systems in office environment," IEEE J. Sel. Areas Commun., vol. 27, no. 8, pp. 1488-1499, Oct. 2009.

[17] J. George, P. F. M. Smulders, and M. H. A. J. Herben, "Application of fan-beam antennas for $60 \mathrm{GHz}$ indoor wireless communication," Electron. Lett., vol. 37, no. 2, pp. 73-74, Jan. 2001.

[18] W. Hong, K.-H. Baek, Y. Lee, Y. Kim, S.-T. Ko, "Study and prototyping of practically large-scale mmWave antenna systems for $5 \mathrm{G}$ cellular devices," IEEE Commun. Mag., vol. 52, no. 9, pp. 63-69, Sep. 2014.

[19] C. Q. Scrantom and J. C. Lawson, "LTCC technology: Where we are and where we're going. II," in IEEE MTT-S Int. Microw. Symp. Dig., Feb. 1999, pp. 193-200.
[20] M.-T. Martinez-Ingles, D. P. Gaillot, J. Pascual-García, J.-M. Molina-Garcia-Pardo, M. Liénard, and J.-V. Rodríguez, "Deterministic and experimental indoor $\mathrm{mmW}$ channel modeling," IEEE Antennas Wireless Propag. Lett., vol. 13, pp. 1047-1050, Dec. 2014.

[21] R. Hansen, Phased Array Antennas. New York, NY, USA: Wiley, 1998.

[22] L. Ranzani, N. Ehsan, and Z. Popović, "G-band frequency-scanned antenna arrays," in Proc. IEEE Antennas Propag. Soc. Int. Symp., Toronto, ON, Canada, Jul. 2010, pp. 1-4.

[23] W. F. Moulder, W. Khalil, and J. L. Volakis, "60-GHz two-dimensionally scanning array employing wideband planar switched beam network," IEEE Antennas Wireless Propag. Lett., vol. 9, pp. 818-821, 2010.

1323 1324 1325

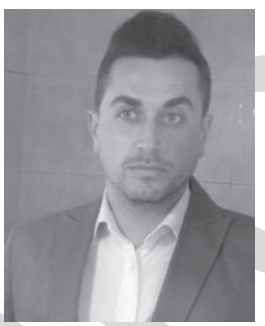

Marc Imbert ( $\mathrm{S}^{\prime}-$ ) was born in Calella (Barcelona) Spain. He received the Telecommunications Engineering degree, Master of Research on Information and Communications Technologies, and the $\mathrm{Ph} . \mathrm{D}$. degree from the Universitat Politècnica de Catalunya (UPC), Barcelona, in 2010, 2013, and 2016, respectively.

In 2009, he joined the Electromagnetic and Photonics Engineering Group (EEF), Department of Signal Theory and Communications, UPC, where he was involved in metamaterial research and its application to antennas. From 2010 to 2011, he was with the Urbiotica S.L, where he was responsible for electromagnetic analysis and antenna design. He was with the Department of Signal Theory and Communications, UPC, where he was involved in the frame of millimeter-wave antennas for communications, radar, and imaging, under the supervision of Professor Jordi Romeu. He was a Visiting Scholar with the University of California, Irvine, CA, USA, where he was with the High-Frequency Electronics Laboratory, under the supervision of Professor Franco De Flaviis. He is currently an RF/Antenna Engineer with the Ficosa Corporation, an automotive industry. His current research interests include metamaterial applications at microwave frequencies, lens antennas, embedded antennas, and electromagnetic characterization of small antennas.

Dr. Imbert was a recipient of the Spanish Government FPI fellowship, and finalist for the 2014 Best Paper Award in Antenna Applications at the European Conference on Antennas and Propagation.

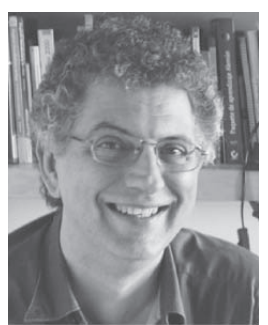

Jordi Romeu (F'12) was born in Barcelona, Spain, in 1962. He received the Ingeniero de Telecomunicación and Doctor Ingeniero de Telecomunicacíon degree from the Universitat Politècnica de Catalunya (UPC), Barcelona, Spain, in 1986 and 1991, respectively.

He has been with the Electromagnetic and Photonic Engineering Group, Signal Theory and Communications Department, UPC, since 1985, where he is currently a full Professor, where he is involved in the research of antenna near-field measurements, antenna diagnostics, and antenna design. He joined the Antenna Laboratory, University of California, Los Angeles, CA, USA, in 1999, as a Visiting Scholar, under the North Atlantic Treaty Organization Scientific Program Scholarship. In 2004, he joined the University of California, Irvine, CA, USA. He has authored 50 refereed papers in international journals and 50 conference proceedings and holds several patents.

Dr. Romeu received the Grand Winner of the European IT Prize by the European Commission, for his contributions in the development of fractal antennas in 1998. 


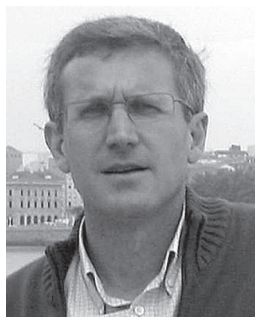

Mariano Baquero-Escudero (S'87-M'90) was born in Murcia, Spain, in 1962. He received the Telecommunications Engineering degree from the Polytechnic University of Catalonia (UPC), Barcelona, Spain, in 1986, and the Ph.D. degree from the Universitat Politècnica de València (UPV), València, Spain, in 1994.

From 1986 to 1988, he was with the Antennas, Microwave and Radar Group, UPC, where he was involved in the development of a cylindrical nearfield facility to measure a 3-D radar antenna in CESELSA. Since 1989, he has been with the UPV, where he was a full Professor in 2003. During 1995, he held a Post-Doctoral grant with the Joint Research Center, European Commission, Ispra, Italy, where he developed high-resolution algorithms for radar applications. From 1996 to 1998, he was a Vice-Dean of the Telecommunications Engineering School of Valencia, Valencia. He is currently with the Communications Department, Institute of Telecommunications and Multimedia Application, UPV. His current research interests include microwave circuit and antenna analysis, design, and measurement.

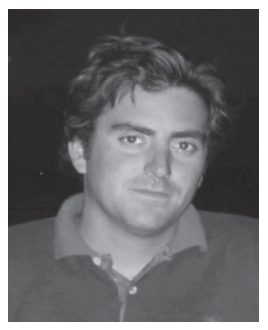

Jose-Maria Molina-Garcia-Pardo received the Engineer of Telecommunications degree from the Universidad Politécnica de Valencia, Valencia, Spain, in 2000 , the M.Sc. degree in communication and signal processing in Newcastle upon Tyne, U.K. in 2001, and the Ph.D. degree in telecommunications from the Universidad Politécnica de Cartagena (UPCT), Cartagena, Spain, in 2004.

In 2001, he joined the Information Technologies and Communications Department, UPCT, where he was an Associate Professor in 2007 and has been a Full Professor since 2016. He is currently a Leader of the SICOMO Research Group. He is the Lead Researcher in some national projects, and participates actively in the European COST action IC-1004 (Radio Communications for Green Smart Environments). He has authored papers that have appeared in over 60 journals indexed in the JCR, over 100 international conferences, and three book chapters. His current research interests include centered on radio-communications, propagation, channel modeling, and experimental channel sounding in different frequency band $(400 \mathrm{MHz}-60 \mathrm{GHz})$, and technologies (GSM, UMTS, LTE, WiFi, WSN, TETRA, millimeterwave, OFDM, MIMO, and cognitive radio).

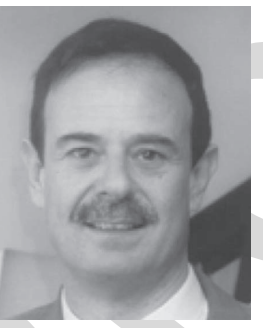

Lluis Jofre (S'79-M'83-SM'07-F'10) was born in Canet de Mar, Spain, in 1956. He received the M.Sc. (Ing.) and Ph.D. (Doctor Ing.) degrees in electrical engineering (telecommunication engineering) from the Technical University of Catalonia (UPC), Barcelona, Spain, in 1978 and 1982, respectively.

$\mathrm{He}$ was a Research Assistant with the Electrophysics Group, UPC, from 1979 to 1980, where he was involved in the analysis and near-field measurement of antennas and scatterers. From 1981 to 1982, he was with the École Supérieure d'Electricité Paris, Gif-sur-Yvette, France, where he was involved in microwave antenna design and imaging techniques for medical and industrial applications. Since 1982, he has been with the Communications Department, Telecommunication Engineering School, UPC, as an Associate Professor and then as a full Professor since 1989. From 1986 to 1987, he was a Visiting Fulbright Scholar with the Georgia Institute of Technology, Atlanta, GA, USA, where he was involved in antennas and electromagnetic imaging and visualization. From 1989 to 1994, he was the Director of the Telecommunication Engineering School, UPC, and from 1994 to 2000, he was the UPC Vice-Rector for academic planning. From 2000 to 2001, he was a Visiting Professor with the Electrical and Computer Engineering Department, Henry Samueli School of Engineering, University of California, Irvine, CA, USA. From 2002 to 2004 he was the Director of the Catalan Research Foundation, and since 2003, he has been the Director of the UPC-Telefonica Chair and the Director of the Promoting Engineering Catalan Program EnginyCAT. He has authored more than 100 scientific and technical papers, reports, and chapters in specialized volumes. His current research interests include antennas, electromagnetic scattering and imaging, system miniaturization for wireless, and sensing industrial and bio-applications.

Dr. Jofre is a member of Different Higher Education Evaluation Agencies at Spanish and European level. Since 2010, he has been the General Director of Universities in the Economy and Knowledge Council of the Catalan Government. 


\section{AUTHOR QUERIES}

\section{AUTHOR PLEASE ANSWER ALL QUERIES}

PLEASE NOTE: We cannot accept new source files as corrections for your paper. If possible, please annotate the PDF proof we have sent you with your corrections and upload it via the Author Gateway. Alternatively, you may send us your corrections in list format. You may also upload revised graphics via the Author Gateway.

AQ:1 = Please provide the section number which represents the last section.

AQ:2 = Please provide the section numbers which represent the following sections.

AQ:3 = Please provide the section number which represents the previous section.

AQ:4 = Please provide the section number which represents the previous section.

AQ:5 = Please provide the section numbers which represent the previous sections.

AQ:6 = Please provide the section numbers which represent the following sections.

AQ:7 = Please provide the expansion for the acronyms "VNA, SISO, SWBA, and PVC."

$\mathrm{AQ}: 8$ = Please provide the section numbers which represent the following sections.

$\mathrm{AQ}: 9=$ Please provide the section numbers which represent the previous sections.

AQ:10 = Please provide the section numbers which represent the previous sections.

AQ:11 = Please provide the section number which represents the last section.

AQ:12 = Please provide the section number which represent the final paper section.

AQ:13 = Please provide the section numbers which represent the previous sections.

AQ:14 = Please provide the section numbers which represent the previous sections.

AQ:15 = Please note that there were discrepancies between the accepted pdf

[imbert.et.al.TAP_special_issue_5G_R2.pdf] and the [FINAL VERSION.docx] in the references. We have followed [FINAL VERSION.docx].

AQ:16 = Please provide the missing IEEE membership year for the author "Marc Imbert."

AQ:17 = Please provide the locations for "Urbiotica S.L, Ficosa Corporation, SICOMO Research Group,

Catalan Research Foundation, and CESELSA." 